\title{
Global Classification of a class of Cubic Vector Fields whose canonical regions are period annuli
}

\author{
M. Caubergh, J. Llibre and J. Torregrosa \\ Departament de Matemàtiques. Edifici C. 08193 Bellaterra (Barcelona) \\ leen@mat.uab.cat,jllibre@mat.uab.cat,torre@mat.uab.cat
}

\begin{abstract}
We study cubic vector fields with inverse radial symmetry, i.e., of the form $\dot{x}=\delta x-y+a x^{2}+$ $b x y+c y^{2}+\sigma(d x-y)\left(x^{2}+y^{2}\right), \dot{y}=x+\delta y+e x^{2}+f x y+g y^{2}+\sigma(x+d y)\left(x^{2}+y^{2}\right)$, having a center at the origin and at infinity; we shortly call them cubic irs-systems. These systems are known to be Hamiltonian or reversible. Here we provide an improvement of the algorithm that characterizes these systems and we give a new normal form.

Our main result is the systematic classification of the global phase portraits of the cubic Hamiltonian irs-systems respecting time (i.e. $\sigma=1$ ) up to topological and diffeomorphic equivalence. In particular there are 22 (resp. 14) topologically different global phase portraits for the Hamiltonian (resp. reversible Hamiltonian) irs-systems on the Poincaré disc.

Finally we illustrate how to generalize our results to polynomial irs-systems of arbitrary degree. In particular we study the bifurcation diagram of a 1-parameter subfamily of quintic Hamiltonian irs-systems. Moreover we indicate how to construct a concrete reversible irs-system with a given configuration of singularities respecting their topological type and separatrix connections.
\end{abstract}

Keywords: classification of global phase portraits, characterization of cubic centers, Lyapunov quantities, Hamiltonian planar vector fields, cubic vector fields.

\section{Introduction}

Let $P$ and $Q$ be two real polynomials in the variables $x$ and $y$, then we say that $X=(P, Q): \mathbb{R}^{2} \longrightarrow \mathbb{R}^{2}$ is a planar polynomial vector field of degree $d$ if the maximum of the degrees of the polynomials $P$ and $Q$ is $d$. Such vector fields are called quadratic or cubic if $d=2$ or $d=3$, respectively. The polynomial differential system associated to the vector field $X$ is

$$
\dot{x}=P(x, y), \quad \dot{y}=Q(x, y) .
$$

Two of the main classical problems in the qualitative theory of real planar polynomial vector fields are the determination of their limit cycles and the center-focus problem; i.e. to distinguish whether a singular point is either a focus or a center. A center is a singular point having a neighborhood fulfilled of periodic orbits with the unique exception of the singular point.

The classification of the centers of the polynomial vector fields is an old problem which started with the quadratic ones by the works of Dulac [1908], Kapteyn [1911, 1912], Bautin [1954], Żołądek [1994b],...; an update on the quadratic centers can be found in [Schlomiuk, 1993a]. For the quadratic polynomial vector fields the characterization of centers is completed. There exist many partial results for the centers of polynomial vector fields of degree larger than 2 , but we are very far to obtain a complete classification of the centers for the class of all polynomial vector fields of degree 3 . In particular the centers of the cubic polynomial vector fields of the form $\dot{x}=-y+P_{3}(x, y), \dot{y}=x+Q_{3}(x, y)$ with $P_{3}$ and $Q_{3}$ homogeneous 
polynomials of degree 3, have been classified by Vulpe \& Sibirskiü [1988], Lloyd \& Pearson [1999] and Żoładek [1994a]. In case former cubic systems are reversible through a linear involution and have a fixed set of dimension 1, a classification of their global phase portraits can be found in [Buzzi et al., 2009].

In this paper we study real planar cubic polynomial systems for which the coefficients in the asymptotic expansion of the Poincaré map both near the origin and near infinity are polynomial in the parameter. These systems are of the form

$$
\begin{aligned}
& \dot{x}=\delta x-y+a x^{2}+b x y+c y^{2}+\sigma(d x-y)\left(x^{2}+y^{2}\right), \\
& \dot{y}=x+\delta y+e x^{2}+f x y+g y^{2}+\sigma(x+d y)\left(x^{2}+y^{2}\right),
\end{aligned}
$$

as also was found in [Blows \& Rousseau, 1993]. Recall that system (1) has a center at infinity if the origin of system (1) is a center after the transformation $x=\cos \theta / r$ and $y=\sin \theta / r$.

First we improve the algorithm used in [Blows \& Rousseau, 1993] to characterize these systems having simultaneously a center at the origin and at infinity. As a result we find a new normal form classifiying these systems as a Hamiltonian and a reversible class (see Theorem 1). Although the center-focus problem has its own interest, the knowledge of the center conditions near the origin (resp. infinity) also is used to study the maximum number of large amplitude limit cycles (resp. small amplitude limit cycles) by ways of the so-called division-derivation algorithm. In [Blows \& Rousseau, 1993] the simultaneous cyclicity problem is also studied and it is proved that the maximum of limit cycles which can appear by simultaneous bifurcation at the origin and at infinity is seven. Some results on the number of large amplitude limit cycles for this class of cubic planar vector fields can be found in [Liu \& Chen, 2002], [Liu \& Huang, 2006] and [Zhang \& Liu, 2006]. More precisely they give concrete bifurcations for which seven large amplitude limit cycles appear. This problem of large amplitude limit cycles is also studied in [Huang \& Liu, 2004a], [Zhang et al., 2006] and [Zhang \& Liu, 2007] for quintic planar vector fields, in [Huang \& Liu, 2004b] for septic planar vector fields, in [Caubergh \& Dumortier, 2008] for classical Liénard systems of even degree and in [Luca et al., 2009] for certain subclasses of generalized Liénard systems.

After rescaling the parameter $\sigma$ can be supposed to be \pm 1 . Written in polar coordinates the leading terms of system (1) at infinity are the same as the ones at the origin, up to the sign of $\sigma$. Hence, the local phase portrait at infinity is topologically equivalent to the one at the origin (after time reversal for $\sigma=-1$.). Therefore a cubic system of type (1) is called a cubic system with inverse radial symmetry or shortly cubic irs-system. If $\sigma>0$ (resp. $\sigma<0$ ) we say that system (1) is a cubic system with inverse radial symmetry respecting time (resp. reversing time).

Theorem 1. The cubic irs-system (i.e., system (1)) has a center at the origin and at infinity if and only if $\delta=d=0$ and it is Hamiltonian or reversible, after a rotation, with respect to the change $(x, y, t) \rightarrow(x,-y,-t)$. That is, after rotation, system (1) satisfies $\delta=d=0, b=-2 g$ and $f=-2 a$ for the Hamiltonian class or $\delta=a=c=d=f=0$ for the reversible class, given below in (2) and (8) respectively.

Next we classify the global phase portraits of the Hamiltonian cubic irs-systems respecting time with respect to topological and diffeomorphic equivalence (see Theorems 2, 3, 4 and 5). The classification of global phase portraits of other Hamiltonian systems is done before, see e.g., [Gasull et al., 2000] and [Guillamon \& Pantazi, 2008]. However their techniques does not apply to the cubic irs-system we consider in this paper. Here we introduce a new systematic technique to systematically study the global phase portraits of Hamiltonian irs-systems.

By Lemma 2 we only need to consider $a=0, g \geq 0$ and $c \geq 0$. Next result details the corresponding phase portrait in each region of the parameter space.

Theorem 2. In Table 1 the global phase portraits of the Hamiltonian cubic irs-systems respecting time, i.e.

$$
X_{(g, c, e)}^{H} \leftrightarrow\left\{\begin{array}{l}
\dot{x}=-y-2 g x y+c y^{2}-y\left(x^{2}+y^{2}\right), \\
\dot{y}=x+e x^{2}+g y^{2}+x\left(x^{2}+y^{2}\right),
\end{array}\right.
$$

are classified up to diffeomorphic equivalence. In Case 2 the explicit expressions of the polynomials $\widehat{R}_{2}$ and $\widehat{R}_{6}$ in $(g, c)$ are given in (45). In Case 4 the parameter $(\alpha, \beta)$ is defined by $(46)$ with $\lambda=-e+2 g$; 
the semi-algebraic subsets $\mathcal{A}, \mathcal{B}, \mathcal{C}, \mathcal{D}, \mathcal{E}, \mathcal{F}, \mathcal{G}, \mathcal{H}$ of $\{(\alpha, \beta): 0 \leq-\beta<\alpha\}$ are defined on page 27 and the figures show the bifurcation with respect to the bifurcation parameter $\lambda$.

Note that Table 1 provides all the phase portraits of system $X_{(g, c, e)}^{H}$ according to values of its parameters. Moreover observe that in cases 1,2 and 3 the classification is done using only the initial parameters of the system. In the study of case 4 there need to be introduced auxiliary parameters in function of the initial ones, which simplifies the classification, see for more details Theorem 7 .

Table 1. Classification of the phase portraits of the Hamiltonian cubic irs-systems respecting time, $X_{(g, c, e)}^{H}$, with respect to diffeomorphic equivalence (Theorem 2).

\begin{tabular}{lll}
\hline & $g<1$ & Figure 10(a) \\
& $g=1$ & Figure 10(b) \\
& $g>1$ & Figure 10(c) \\
\hline & $\widehat{R}_{2}<0$ & Figure 12(a) \\
& $\widehat{R}_{2}=0$ & Figure 12(b) \\
& $g<1, \widehat{R}_{2}>0$ & Figure 12(c) \\
& $g=1$ & Figure 12(d) \\
& $g>1, \widehat{R}_{6}<0$ & Figure 12(e) \\
& $\widehat{R}_{6}=0$ & Figure 12(f) \\
& $\widehat{R}_{6}>0$ & Figure 12(g) \\
\hline & $e<2$ & Figure 13(a) \\
& $e=2: c \neq 0, e=2 g$ & Figure 13(b) \\
& $e>2$ & Figure 13(c) \\
& $(\alpha, \beta) \in \mathcal{A}$ & Theorem 7(1), Figure 17 \\
& $(\alpha, \beta) \in \mathcal{B}$ & Theorem 7(2), Figure 18 \\
& $(\alpha, \beta) \in \mathcal{C}$ & Theorem 7(3), Figure 19 \\
& $(\alpha, \beta) \in \mathcal{D}$ & Theorem 7(4), Figure 20 \\
& $(\alpha, \beta) \in \mathcal{E}$ & Theorem 7(5), Figure 21 \\
& $(\alpha, \beta) \in \mathcal{F}$ & Theorem 7(6), Figure 22 \\
& $(\alpha, \beta) \in \mathcal{G}$ & Theorem 7(7), Figure 23 \\
& $(\alpha, \beta) \in \mathcal{H}$ & Theorem 7(8), Figure 24 \\
\hline
\end{tabular}
alence.

In Theorem 3 we classify all phase portraits for Hamiltonian cubic irs-systems up to topological equiv-

Theorem 3. The phase portrait of a Hamiltonian cubic irs-system, $X_{(g, c, e)}^{H}$, is topologically equivalent to one of the 22 phase portraits described in Figure 1.

Theorem 4. The phase portrait of a reversible Hamiltonian cubic irs-system respecting time, $X_{(g, 0, e)}^{H}$, is topologically equivalent to one of the 14 phase portraits presented in Figure 2. Furthermore the phase portrait of these cubic reversible Hamiltonian systems is uniquely determined with respect to topological equivalence in case that the total number of singularities equals 1,2, 4 or 6.

Throughout this paper we will use the notation $|\cdot|$ for the Euclidean norm on $\mathbb{R}^{2}$ as well for the absolute value of a real number.

Theorem 5. The global phase portrait of a Hamiltonian cubic irs-system respecting time, $X_{(g, c, e)}^{H}$ has at most 7 singularities of which one is the center at the origin. Furthermore the following statements hold:

(1) There are at most 3 distinct straight lines $l$ through the origin that carry 2 or more singularities of (1). Denote these lines by $l_{i}, 0 \leq i \leq k, k \leq 2$.

(2) If $l_{i}(0 \leq i \leq k, k \leq 2)$ is such a straight line through the origin, then $l_{i}$ carries at most 2 singularities different from the origin. 


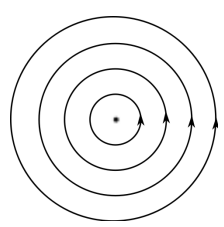

(I)

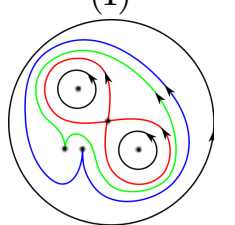

(Va)

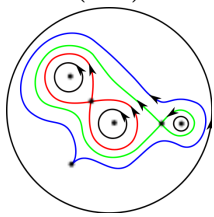

(VIa)

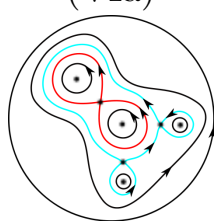

(VIIe)

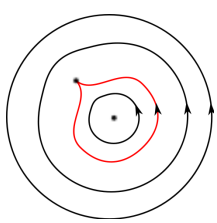

(II)

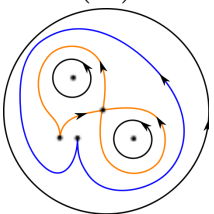

(Vb)

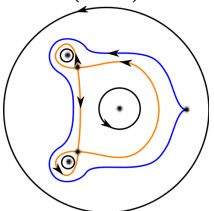

(VIb)

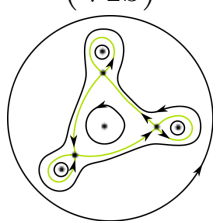

(VIIf)

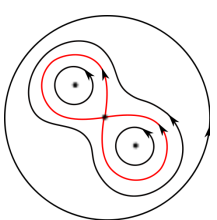

(IIIa)

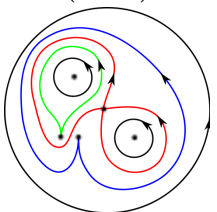

(Vc)

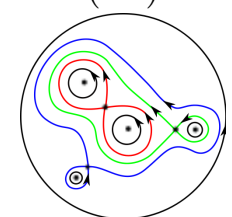

(VIIa)

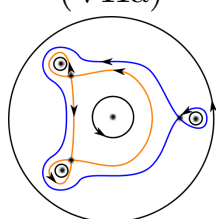

(VIIg)
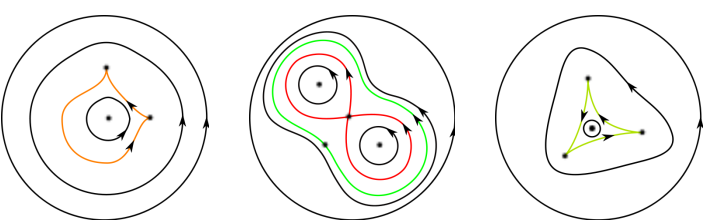

(IIIb)

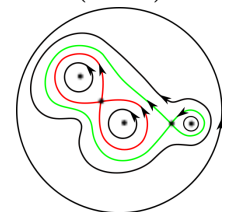

(IVa)

(IVb)
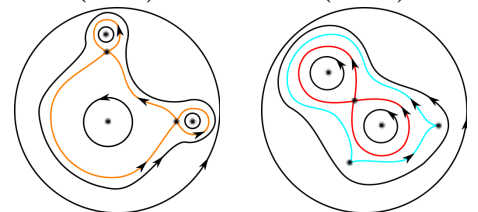

(Vd)

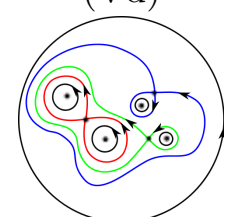

(Ve)

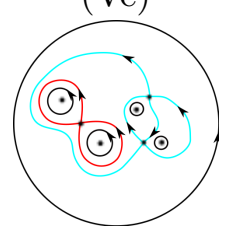

(VIIc)

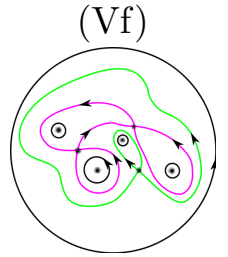

(VIId)

Fig. 1. Topologically different phase portraits of the cubic Hamiltonian irs-systems (see Theorem 3).

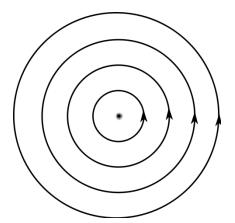

(I)

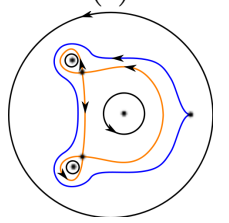

(VI)

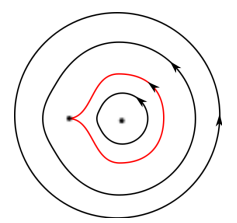

(II)

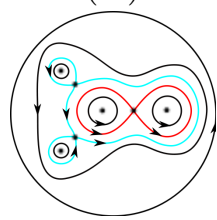

(VIIa)

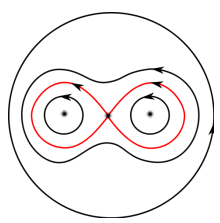

(IIIa)

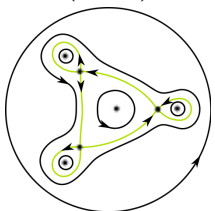

(VIIb)

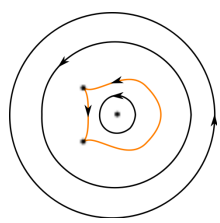

(IIIb)

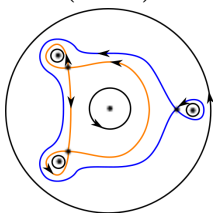

(VIIc)

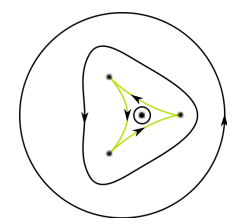

(IV)

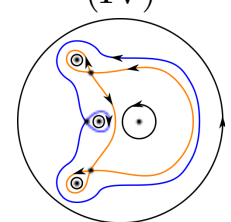

(VIId)

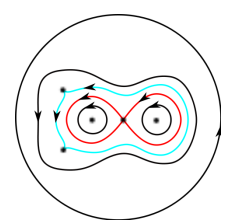

(Va)

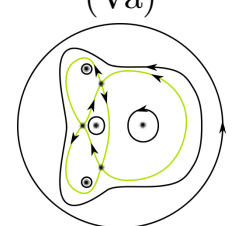

(VIIe)

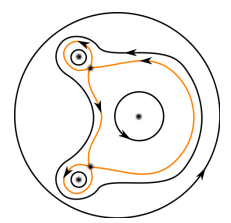

$(\mathrm{Vb})$

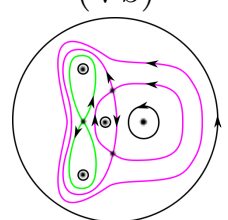

(VIIf)

Fig. 2. Topologically different phase portraits of the cubic reversible Hamiltonian irs-systems (see Theorem 4).

(a) When $l_{i}$ carries 1 singularity different from the origin, then we denote this singularity by $s^{i}$ and we have $\left|s^{i}\right|=1$. Furthermore its Hamiltonian value is $h^{i} \equiv 1 / 12$.

(b) When $l_{i}$ carries 2 singularities different from the origin, then both singularities lie on the same side of the origin and we denote them by $s_{ \pm}^{i}$ having $\left|s_{-}^{i}\right|<1<\left|s_{+}^{i}\right|$. Furthermore $h_{-}^{i}<h_{+}^{i}<1 / 12$, where $h_{ \pm}^{i}$ denotes the Hamiltonian value at $s_{ \pm}^{i}$ respectively.

(3) Assume that there are $k+1$ straight lines $(k \leq 2)$, say $l_{i}, 0 \leq i \leq k$, carrying at least two singularities, then we order these lines such that $h_{-}^{i_{1}} \leq h_{-}^{i_{2}}$ for $0 \leq i_{1} \leq i_{2} \leq k$, where we extend the notation $h_{-}^{i} \equiv h^{i}$ in case $l_{i}$ carries only 1 singularity outside of the origin.

(4) A Hamiltonian cubic irs-system has maximally 7 disjoint period annuli.

(5) The global phase portraits can be classified up to topological equivalence in terms of the number of 
singularities $\left(\#_{s}\right)$ and the order of their values of the Hamiltonian. For three configurations of the Hamiltonian values an additional algebraic condition has to be checked to determine the phase portraits uniquely up to topological equivalence. This classification is presented in Table 2.

Table 2. Topological classification of the phase portraits of the cubic Hamiltonian systems based on the number of singularities. The number $m(A)$ is defined as the maximal multiplicity of a zero of $A$ on $[0, \pi)$ and $A$ and $\Pi$ are respectively defined in (12) and (52).

\begin{tabular}{|c|c|c|c|}
\hline$\# s$ & Hamiltonian values & Condition & Figure \\
\hline 1 & & & Figure 1(I) \\
\hline 2 & $h^{0}=1 / 12$ & & Figure 1(II) \\
\hline 3 & $h^{0}=h^{1}=1 / 12$ & & Figure 1(IIIa) \\
\hline 3 & $h_{-}^{0}<1 / 12$ & & Figure 1(IIIb) \\
\hline 4 & $h_{-}^{0}<h^{1}=1 / 12$ & & Figure $1(\mathrm{IVa})$ \\
\hline 4 & $h^{0}=h^{1}=h^{2}=1 / 12$ & & Figure 1(IVb) \\
\hline 5 & $h_{-}^{0}<h_{+}^{1}<h_{-}^{1}<1 / 12$ & $m(A)=2$ & Figure $1(\mathrm{Va})$ \\
\hline 5 & $h_{-}^{0}=h_{+}^{1}<h_{-}^{1}<1 / 12$ & $m(A)=2$ & Figure $1(\mathrm{Vb})$ \\
\hline 5 & $h_{+}^{1}<h_{-}^{0}<h_{-}^{1}<1 / 12$ & $m(A)=2$ & Figure $1(\mathrm{Vc})$ \\
\hline 5 & $h_{-}^{0}<h_{-}^{1}<1 / 12$ & $m(A)=1$ & Figure $1(\mathrm{Vd})$ \\
\hline 5 & $h_{+}^{0}=h_{+}^{1}<h_{-}^{0}=h_{-}^{1}<1 / 12$ & & Figure $1(\mathrm{Ve})$ \\
\hline 5 & $h_{-}^{0}<h^{1}=h^{2}=1 / 12$ & & Figure 1(Vf) \\
\hline 6 & $h_{-}^{0}<h_{-}^{1}<h^{2}=1 / 12$ & & Figure $1(\mathrm{VIa})$ \\
\hline 6 & $h_{+}^{0}=h_{+}^{1}<h_{-}^{0}=h_{-}^{1}<h^{2}=1 / 12$ & & Figure 1(VIb) \\
\hline 7 & $h_{-}^{0}<h_{-}^{1}<h_{-}^{2}<1 / 12$ & $\Pi>0$ & Figure 1(VIIa) \\
\hline 7 & $\begin{array}{l}h_{-}^{0}<h_{-}^{1}<h_{-}^{2}<1 / 12 \\
\text { and } h_{+}^{2} \notin\left\{h_{-}^{0}, h_{-}^{1}\right\}\end{array}$ & $\Pi<0$ & Figure 1 (VIIb) \\
\hline 7 & $\left\{\begin{array}{l}h_{-}^{0}<h_{-}^{1}=h_{+}^{2}<h_{-}^{2}<1 / 12 \text { or } \\
h_{+}^{2}<h_{-}^{1}=h_{-}^{0}<h_{-}^{2}<1 / 12\end{array}\right.$ & $\Pi<0$ & Figure 1(VIIc) \\
\hline 7 & $\left\{\begin{array}{l}h_{-}^{0}=h_{+}^{2}<h_{-}^{1}<h_{-}^{2}<1 / 12 \text { or } \\
h_{-}^{0}=h_{-}^{1}<h_{+}^{2}<h_{-}^{2}<1 / 12\end{array}\right.$ & $\Pi<0$ & Figure 1 (VIId) \\
\hline 7 & $h_{-}^{0}<h_{-}^{1}=h_{-}^{2}<1 / 12$ & & Figure 1(VIIe) \\
\hline 7 & $h_{-}^{0}=h_{-}^{1}=h_{-}^{2}<1 / 12$ & & Figure 1(VIIf) \\
\hline 7 & $h_{-}^{0}=h_{-}^{1}<h_{-}^{2}<1 / 12$ & $\Pi>0$ & Figure $1(\mathrm{VIIg})$ \\
\hline 7 & $h_{-}^{0}=h_{-}^{1}=h_{+}^{2}<h_{-}^{2}<1 / 12$ & $\Pi<0$ & Figure 1(VIIh) \\
\hline
\end{tabular}

The paper is organized as follows. In section 2 we prove Theorem 1. Using the improved algorithm of Gasull \& Torregrosa [2001] to calculate Lyapunov quantities we derive a new normal form for the cubic irs-systems. In section 3 we recall basic notions and results on singular points, on the infinity and equivalent vector fields. Next in section 4 we study the qualitative properties of the 4-parameter Hamiltonian subfamily for $\sigma=1$ in polar coordinates. This leads to the introduction of trigonometric polynomials $A$ and $B$ that play an important role in the description of the bifurcation diagram of the phase portraits (see subsection 4.1). Next in subsection 4.2 we reduce the study of the 4-parameter Hamiltonian family to the one of a 3-parameter Hamiltonian subfamily. Next in subsection 4.3 we analyze the singularities along a 'ray' depending on its multiplicity and in subsection 4.4 we study the properties of the Hamiltonian. The local analysis of the singularities and the properties of the Hamiltonian enable us to classify the global phase portraits of the Hamiltonian class up to diffeomorphic equivalence. For $\sigma=1$ the bifurcation of the global phase portraits are described in section 5 for arbitrary but fixed number of the rays that can carry singularities multiplicity taken into account (sections 5.1, 5.2, 5.3 and 5.4). These characterizations prove Theorems 2 and 5 . The proof of Theorem 3 follows by identifying the topologically nonequivalent phase portraits of Figures 10, 12, 13, 17, 18, 19, 20, 21, 22, 23 and 24; the proof of Theorem 4 follows from Theorem 3 by considering the ones appearing for $c=0$. Finally, in section 6 , using the systematic method 
previously introduced for these cubic systems, we construct concrete examples of polynomial Hamiltonian and reversible systems of higher degree than 3 with a given configuration of singularities and connections of the separatrices. In a forthcoming paper we treat the reversible class.

\section{Normal Forms for Cubic irs-Systems}

In this section we deduce a normal form for the cubic irs-systems. Ending the writing of this paper we found that the centers for these systems also are characterised in [Blows \& Rousseau, 1993] by a calculation of Lyapunov quantities. However in [Blows \& Rousseau, 1993] calculations are performed at infinity while here we perform calculations near the origin parallel with calculations at infinity. Moreover we include an improvement of the algorithm as introduced in [Gasull \& Torregrosa, 2001] what lead to a significant reduction of the calculations. Since our proof is shorter and leads to a different normal form we include it here.

Lyapunov quantities are defined by an algebraic lemma in [Schlomiuk, 1993b] for a weak focus; we recall a slightly generalized version of it which proof can be found in [Caubergh \& Dumortier, 2004].

Lemma 1 [Lyapunov quantities]. Let $\left(X_{\lambda}\right), \lambda \in \mathcal{W} \subset \mathbb{R}^{p}$ be an analytic family of planar vector fields with 1 -jet $(d(\lambda) x-c(\lambda) y) \frac{\partial}{\partial x}+(c(\lambda) x+d(\lambda) y) \frac{\partial}{\partial y}$, for some analytic functions $c, d: \mathcal{W} \rightarrow \mathbb{R}$ with $c(\lambda) \neq 0$ for all $\lambda$. Then there exists a formal power series $F_{\lambda}$ with

$$
F_{\lambda}(x, y)=\frac{1}{2}\left(x^{2}+y^{2}\right)+O\left(\|(x, y)\|^{2}\right), \quad\|(x, y)\| \rightarrow 0,
$$

and there exist analytic functions $V_{i}, i \in \mathbb{N}$ such that

$$
X_{\lambda} F_{\lambda}(x, y)=\sum_{i=0}^{\infty} V_{i}(\lambda)\left(x^{2}+y^{2}\right)^{i+1} .
$$

Furthermore such analytic functions $\left\{V_{i}, i \in \mathbb{N}\right\}$ are uniquely determined by (3) in the sense that if another set of analytic functions $\left\{W_{i}, i \in \mathbb{N}\right\}$ satisfies (3), then

$$
W_{i}=V_{i} \bmod \left(V_{0}, V_{1}, \ldots, V_{i-1}\right),
$$

where $\left(V_{0}, V_{1}, \ldots, V_{i-1}\right)$ denotes the ideal generated by the analytic functions $V_{j}, 0 \leq j \leq i-1$. Any set of analytic functions satisfying (3) is called a set of Lyapunov quantities for $X_{\lambda}$.

The systems $X_{\lambda}, \lambda \in \mathcal{W}$ having a center at the origin can be characterized by the vanishing of all Lyapunov quantities: if $\left\{V_{i}, i \in \mathbb{N}\right\}$ is a set of Lyapunov quantities of $X_{\lambda}$, then the focus at the origin of $X_{\lambda_{0}}$ is a center if and only if $V_{i}\left(\lambda_{0}\right)=0$ for all $i \in \mathbb{N}$. In particular the systems $X_{\lambda}, \lambda \in \mathcal{W}$ having a weak focus at the origin can be characterized by the vanishing of the 0th Lyapunov quantity of $X_{\lambda}$.

In practice Lyapunov quantities can be calculated by putting the system in polar coordinates $(r, \theta)$

$$
\frac{d r}{d \theta}=\sum_{k=1}^{\infty} S_{k}(\theta) r^{k},
$$

where $S_{k}(\theta)$ are homogeneous trigonometric polynomials of degree $k$ whose coefficients are polynomials in $(a, b, c, d, e, f, g, \delta, \sigma)$.

Denote by $r\left(\theta, r_{0}\right)$ the solution of (4) such that $r=r_{0}$ when $\theta=0$. In this case and for $r$ small enough we can write

$$
r\left(\theta, r_{0}\right)=r_{0}+\sum_{k=1}^{\infty} u_{k}(\theta) r_{0}^{k},
$$

for analytic functions $u_{k}$ with $u_{k}(0)=0$ for $k \geq 1$. The Poincaré return map is defined as

$$
\Pi\left(r_{0}\right)=r\left(2 \pi, r_{0}\right)=r_{0}+\sum_{k=1}^{\infty} u_{k}(2 \pi) r_{0}^{k} .
$$


In particular if $\left\{V_{i}, i \in \mathbb{N}\right\}$ is a set of Lyapunov quantities for (1), then

$$
V_{0}=c_{0} \delta \text { and } V_{i}=c_{i} u_{2 i+1}(2 \pi) \bmod \left(V_{0}, V_{1}, \ldots, V_{i-1}\right)
$$

for some non-zero constants $c_{i}, i \geq 0$ (see [Caubergh \& Dumortier, 2004]). Therefore to find the center conditions, it suffices to determine $v_{k}=0, k \geq 1$ recursively where $v_{1}=u_{1}(2 \pi) \bmod (\delta)$ and $v_{k}=$ $u_{2 k+1}(2 \pi) \bmod \left(\delta, u_{3}(2 \pi), u_{5}(2 \pi), \ldots, u_{2 k-1}(2 \pi)\right)$ for $k \geq 1$.

By calculating the set $v_{k}, k \geq 1$ instead of $V_{k}, k \geq 1$ computations and computer time are significantly reduced; if one in addition works in complex notation as does the algorithm introduced in [Gasull \& Torregrosa, 2001], the computer time is even more reduced. System (1) can be written in complex coordinates $(z, \bar{z})$, as

$$
\dot{z}=(\delta+i) z+\sum_{k=1}^{\infty} R_{k}(z, \bar{z}) \quad \text { and } \quad R_{k}(z, \bar{z})=\sum_{l=0}^{k} r_{l, k-l} z^{l} \bar{z}^{k-l},
$$

where $r_{l, k-l}$ depend on the parameter $(a, b, c, d, e, f, g, \delta, \sigma)$. Notice that only in this section $\bar{z}$ denotes the conjugate of a complex number $z$ and $i$ the number $\sqrt{-1}$.

Proof. [Proof of Theorem 1]After the change of variables $z=x+i y$ system (1) writes in the form (5) where

$$
\begin{array}{lll}
r_{10}=\delta+i, & r_{20}=(a-c+f+i(-b+e-g)) / 4, & r_{30}=0, \\
r_{01}=0, & r_{21}=\sigma(d+i), \\
& r_{11}=(a+c+i(e+g)) / 2, & r_{12}=0, \\
r_{02}=(a-f-a+i(b+e-g)) / 4, & r_{03}=0 .
\end{array}
$$

Then the cubic irs-system (1) has a weak focus at the origin when $\delta=0$ and after the transformation $x=\cos \theta / r$ and $y=\sin \theta / r$ we can say that system (1) has a weak focus at infinity when $d=0$.

After a rescaling of the variables and time it is not restrictive to assume that $\sigma= \pm 1$. Here we only consider the case $\sigma=1$, the case $\sigma=-1$ is analogous. The computation of the Lyapunov quantities according to the algorithm in [Gasull \& Torregrosa, 2001] gives

$$
\begin{aligned}
v_{1} & =\pi i\left(r_{20} r_{11}-\bar{r}_{20} \bar{r}_{11}\right), \\
v_{2} & =2 \pi i\left(2 \bar{r}_{02} r_{11}^{3}-2 r_{02} \bar{r}_{11}^{3}+3 \bar{r}_{02} \bar{r}_{20} r_{11}^{2}-3 r_{02} r_{20} \bar{r}_{11}^{2}+2 r_{02} r_{20}^{2} \bar{r}_{11}-2 \bar{r}_{02} \bar{r}_{20}^{2} r_{11}\right) / 3, \\
v_{3} & =\pi i\left(-146 \bar{r}_{02} \bar{r}_{20} r_{11}^{2}+146 r_{02} r_{20} \bar{r}_{11}^{2}-72 \bar{r}_{02} r_{11}^{3}+72 r_{02} \bar{r}_{11}^{3}-8 r_{02} r_{20}^{3}\right. \\
& +8 \bar{r}_{20}^{3} \bar{r}_{02}-30 r_{11}^{3} \bar{r}_{02} \bar{r}_{20} \bar{r}_{11}+30 r_{02} \bar{r}_{20} \bar{r}_{11}^{4}-15 r_{11}^{4} \bar{r}_{02} \bar{r}_{11}+15 r_{11} r_{02} \bar{r}_{11}^{4} \\
& \left.-15 r_{02}^{2} \bar{r}_{02} \bar{r}_{11}^{3}+15 r_{11}^{3} \bar{r}_{02}^{2} r_{02}-30 r_{02}^{2} r_{20} \bar{r}_{02} \bar{r}_{11}^{2}+30 r_{11}^{2} r_{02} \bar{r}_{02}^{2} \bar{r}_{20}\right) / 6, \\
v_{4} & =\pi i\left(3926 \bar{r}_{02} \bar{r}_{20} r_{11}^{2}-3926 r_{02} r_{20} \bar{r}_{11}^{2}+2232 \bar{r}_{02} r_{11}^{3}-2232 r_{02} \bar{r}_{11}^{3}+2152 \bar{r}_{20}^{3} \bar{r}_{02}\right. \\
& -2152 r_{02} r_{20}^{3}+105 r_{02}^{2} \bar{r}_{02} \bar{r}_{11}^{3}-105 r_{11}^{3} \bar{r}_{02}^{2} r_{02}+840 r_{02}^{2} r_{20}^{3} \bar{r}_{02}-840 \bar{r}_{02}^{2} \bar{r}_{20}^{3} r_{02} \\
& \left.+13035 r_{11} r_{02} \bar{r}_{11}^{4}-13035 r_{11}^{4} \bar{r}_{02} \bar{r}_{11}+26070 r_{02} \bar{r}_{20} \bar{r}_{11}^{4}-26070 r_{11}^{3} \bar{r}_{02} \bar{r}_{20} \bar{r}_{11}\right) / 225, \\
v_{5} & =16 \pi i\left(-143 \bar{r}_{02} \bar{r}_{20} r_{11}^{2}+143 r_{02} r_{20} \bar{r}_{11}^{2}-66 \bar{r}_{02} r_{11}^{3}+66 r_{02} \bar{r}_{11}^{3}-44 r_{02} r_{20}^{3}+44 \bar{r}_{20}^{3} \bar{r}_{02}\right. \\
& \left.-2890 r_{11}^{3} \bar{r}_{02} \bar{r}_{20} \bar{r}_{11}+2890 r_{02} \bar{r}_{20} \bar{r}_{11}^{4}-1445 r_{11}^{4} \bar{r}_{02} \bar{r}_{11}+1445 r_{11} r_{02} \bar{r}_{11}^{4}\right) / 105,
\end{aligned}
$$

and $v_{6}=v_{7}=0$. Using only the vanishing of the first four Lyapunov quantities we obtain

$$
v_{5}^{2}=0 \bmod \left(v_{1}, v_{2}, v_{3}, v_{4}\right) .
$$

Now we show that $\left\{\delta, d, v_{1}, v_{2}, v_{3}, v_{4}\right\}$ are the Lyapunov quantities that control the centers in real coordinates by adding the condition

$$
v_{4}^{2}=-20480 \pi^{2} r_{11} \bar{r}_{11}^{2}\left(-r_{02} \bar{r}_{11}^{3}+\bar{r}_{02} r_{11}^{3}\right)^{2}\left(2 \bar{r}_{20}+r_{11}\right) \bmod \left(v_{1}, v_{2}, v_{3}\right)
$$

and solving system $\mathcal{S}=\left\{v_{1}=0, v_{2}=0, v_{3}=0, v_{4}=0\right\}$. We solve system $\mathcal{S}$ for the variables $r_{k l}$ and next we return to the original parameters $a, b, c, e, f, g$. By taking into account that system (1) is a real 
differential system we obtain some concrete families that are of Hamiltonian or reversible type. Then we can conclude that system (1) has a center at the origin if and only if the Lyapunov quantities $v_{j}, 0 \leq j \leq 4$ are zero.

Notice that these two classes are invariant after a rotation with respect to the origin. Up to this rotation the Hamiltonian class is the 4-parameter subfamily within system (1) defined by the conditions

$$
\delta=d=0, b=-2 g \text { and } f=-2 a,
$$

and the Hamiltonian is given by

$$
H(x, y)=\frac{1}{2}\left(x^{2}+y^{2}\right)+\frac{1}{3} e x^{3}-a x^{2} y+g x y^{2}-\frac{1}{3} c y^{3}+\frac{1}{4} \sigma\left(x^{2}+y^{2}\right)^{2} .
$$

The reversible class, up to some rotation with respect to the origin, is the 3-parameter subfamily within system (1) defined by the conditions $\delta=a=c=d=f=0$. Notice that this family is symmetric with respect to the change $(x, y, t) \mapsto(x,-y,-t)$.

In subsection 4.2 for $\sigma=1$ we will see that up to linear conjugacy the phase portraits of the Hamiltonian class are represented by the 3-parameter subfamily $b=-2 g, f=-2 a$ and $a=0$. Then the Hamiltonian class can be represented by the 3 -parameter family defined by (2), i.e.

$$
X_{(g, c, e)}^{H} \leftrightarrow\left\{\begin{array}{l}
\dot{x}=-y-2 g x y+c y^{2}-y\left(x^{2}+y^{2}\right), \\
\dot{y}=x+e x^{2}+g y^{2}+x\left(x^{2}+y^{2}\right),
\end{array}\right.
$$

with Hamiltonian $H=H_{(g, c, e)}$ given by

$$
H(x, y)=\frac{1}{2}\left(x^{2}+y^{2}\right)+\frac{1}{3} e x^{3}+g x y^{2}-\frac{1}{3} c y^{3}+\frac{1}{4}\left(x^{2}+y^{2}\right)^{2},
$$

and the reversible class can be represented by the 3-parameter family

$$
X_{(g, \xi, e)}^{R} \leftrightarrow\left\{\begin{array}{l}
\dot{x}=-y+(\xi-2 g) x y-y\left(x^{2}+y^{2}\right), \\
\dot{y}=x+e x^{2}+g y^{2}+x\left(x^{2}+y^{2}\right) .
\end{array}\right.
$$

Notice that by increasing $|\xi|$ from $\xi=0$, the reversible class is born from the Hamiltonian subclass defined by $c=0$, i.e., $X_{(g, 0, e)}^{R}=X_{(g, 0, e)}^{H}$. These facts are also true for the case $\sigma=-1$.

\section{Topological and Diffeomorphic Equivalence}

In this section we recall notions and basic theorems that we will use in the classification of the phase portraits of the Hamiltonian cubic irs-systems.

We denote by $\mathcal{P}_{n}\left(\mathbb{R}^{2}\right)$ the set of real planar polynomial vector fields of the form $X(x, y)=$ $(P(x, y), Q(x, y))$ where $P$ and $Q$ are real polynomials in the variables $(x, y)$ of degree $n$. We denote by $p(X)$ the Poincaré compactified vector field corresponding to $X \in \mathcal{P}_{n}\left(\mathbb{R}^{2}\right)$ which is an analytic vector field induced on $\mathbb{S}^{2}$, as described in [Gonsales, 1984], or Chapter 5 of [Dumortier et al., 2006]. In this way, $\mathbb{S}^{1}$ is identified to the infinity of $\mathbb{R}^{2}$; in this paper when we speak about infinity, we mean the circle of infinity of $X$. System (1) doesn't have singularities at infinity, so the invariant circle $\mathbb{S}^{1}$ at infinity is a periodic orbit.

We say that two polynomial vector fields $X$ and $Y$ on $\mathbb{R}^{2}$ are topologically equivalent (resp. diffeomorphically equivalent, resp. diffeomorphically linear) if there exists a homeomorphism (resp. diffeomorphism, resp. isomorphism) on $\mathbb{S}^{2}$ preserving the infinity $\mathbb{S}^{1}$ carrying orbits of the flow induced by $p(X)$ into orbits of the flow induced by $p(Y)$. In particular the phase portraits are drawn in the disk $\mathbb{D}^{2}$ that is obtained by projecting the northern hemisphere of the Poincare sphere on the equatorial plane.

We will rely on a result due to Markus [1960], Neumann [1975] and Peixoto [1971] to determine easily whether two diffeomorphically non-equivalent systems are topologically equivalent. To state this result we first recall some definitions from [Dumortier et al., 2006]. 
We say that a flow $\left(\mathbb{R}^{2}, \varphi\right)$ is parallel if it is topologically equivalent to the strip flow (i.e., defined by the flow of $\dot{x}=1, \dot{y}=0$ ), the period annulus or annulus flow (i.e., defined by the flow of $\dot{r}=0, \dot{\theta}=1$ ) or the spiral or nodal flow (i.e., defined by the flow of $\dot{r}=r, \dot{\theta}=0$ ).

The boundary of a maximal open region on $\mathbb{R}^{2}$ on which the flow is parallel is called an extended separatrix skeleton; its structure is precisely described in [Dumortier et al., 2006]. The union $S$ of all orbits in the extended separatrix skeleton is a closed set invariant under the flow. A maximal connected component of $\mathbb{R}^{2} \backslash S$, which is necessarily invariant under the flow, is called a canonical region. Given a flow $\left(\mathbb{R}^{2}, \varphi\right)$ by the completed separatrix skeleton we mean the union of the extended separatrix skeleton of the flow together with one orbit from each of the canonical regions.

Let $C_{1}$ and $C_{2}$ be the completed separatrix skeletons of the flows $\left(\mathbb{R}^{2}, \varphi_{1}\right)$ and $\left(\mathbb{R}^{2}, \varphi_{2}\right)$ respectively. Then we say that $C_{1}$ and $C_{2}$ are topologically equivalent if and only if there exists a homeomorphism from $\mathbb{R}^{2}$ to $\mathbb{R}^{2}$ that maps the orbits of $C_{1}$ to the orbits of $C_{2}$ preserving the orientation.

According to the so-called Markus-Neumann-Peixoto Theorem it suffices to describe the completed separatrix skeleton in order to determine the topological equivalence class of a differential system. More precisely it is stated as follows:

Theorem 6 [Markus-Neumann-Peixoto]. Assume that $\left(\mathbb{R}^{2}, \varphi_{1}\right)$ and $\left(\mathbb{R}^{2}, \varphi_{2}\right)$ are two continuous flows with only isolated singular points. Then these flows are topologically equivalent if and only if their completed separatrix skeletons are equivalent.

\section{The Hamiltonian Class}

\subsection{Polar coordinates}

By (6) the Hamiltonian class is a 4-parameter family formed by the cubic polynomial differential system of the form

$$
X_{(a, g, c, e)} \leftrightarrow\left\{\begin{array}{l}
\dot{x}=-y+a x^{2}-2 g x y+c y^{2}-y\left(x^{2}+y^{2}\right) \\
\dot{y}=x+e x^{2}-2 a x y+g y^{2}+x\left(x^{2}+y^{2}\right)
\end{array}\right.
$$

with Hamiltonian $H=H_{(a, g, c, e)}$ given by

$$
H(x, y)=\frac{1}{2}\left(x^{2}+y^{2}\right)+\frac{1}{3} e x^{3}-a x^{2} y+g x y^{2}-\frac{1}{3} c y^{3}+\frac{1}{4}\left(x^{2}+y^{2}\right)^{2} .
$$

For all values of the 4-dimensional parameter $(a, g, c, e)$ the vector field $X_{(a, g, c, e)}$ has a singularity at the origin; it is of center type. To localize the other singularities of $X_{(a, g, c, e)}$ for some arbitrary but fixed value of $(a, g, c, e)$, and to determine their type, we study this system using polar coordinates, $x=r \cos \theta, y=r \sin \theta$. Thus we obtain:

$$
\begin{aligned}
& \dot{r}=r^{2} A(\theta), \\
& \dot{\theta}=1+r B(\theta)+r^{2},
\end{aligned}
$$

where

$$
\begin{aligned}
& A(\theta)=a \cos ^{3} \theta+(e-2 g) \cos ^{2} \theta \sin \theta+(c-2 a) \cos \theta \sin ^{2} \theta+g \sin ^{3} \theta, \\
& B(\theta)=e \cos ^{3} \theta-3 a \cos ^{2} \theta \sin \theta+3 g \cos \theta \sin ^{2} \theta-c \sin ^{3} \theta .
\end{aligned}
$$

Although the functions $A$ and $B$ depend on the parameter, we often leave it out of our notation to facilitate the reading. Singularities of (9) correspond to values $(r, \theta)$ with

$$
A(\theta)=0, \quad 1+r B(\theta)+r^{2}=0 .
$$

To localize the singularities we look for angles $\theta$ satisfying $A(\theta)=0$, i.e.,

$$
\cos \theta=0 \quad \text { or } \quad a+(b+e) \tan \theta+(c+f) \tan ^{2} \theta+g \tan ^{3} \theta=0 .
$$

Since $\left.A(\theta)\right|_{\cos \theta=0}=0$ is equivalent to $g=0$, there are at most 6 solutions $\theta^{*}$ satisfying $A\left(\theta^{*}\right)=0$, say $\theta_{i}, \theta_{i}+\pi(i=0,1,2)$. For each such $\theta^{*}$ we look for solutions $r>0$ such that $1+r B\left(\theta^{*}\right)+r^{2}=0$. Clearly 
for $\left|B\left(\theta^{*}\right)\right| \leq 2$ this equation has solutions

$$
r_{ \pm}^{*}=\frac{-B\left(\theta^{*}\right) \pm \sqrt{\left(B\left(\theta^{*}\right)\right)^{2}-4}}{2},
$$

which are positive if $B\left(\theta^{*}\right)<0$.

In particular since

$$
r_{+}^{*} r_{-}^{*}=1,
$$

we have

$$
r_{-}^{*}<1<r_{+}^{*} \text { if } B\left(\theta^{*}\right)<-2 \quad \text { and } \quad r_{-}^{*}=r_{+}^{*}=1 \text { if } B\left(\theta^{*}\right)=-2 \text {. }
$$

\subsection{Reduction to 3-parameter subfamily}

From the following lemma it follows that we can restrict our study without loss of generality to the 3parameter subfamily $X_{(g, c, e)}^{H}$ defined in (2), i.e., we can assume that

$$
a=0 .
$$

Moreover we can suppose that $g, c \geq 0$ and that there exists $1 \leq n \leq 3$ such that

$$
A^{(i)}(0)=0, \quad \text { for all } 0 \leq i<n \quad \text { and } \quad A^{(n)}(0) \neq 0 .
$$

Lemma 2. For $a, g, c, e \in \mathbb{R}$ there exist $\bar{g}, \bar{c} \geq 0$ such that the vector field $X_{(a, g, c, e)}$ is linearly equivalent to $X_{(0, \bar{g}, \bar{c}, e)}$ defined in (9). The linear equivalence is given by the composition of a rotation and some of the following symmetry operations:

$$
T_{1}(x, y, t)=(-x,-y, t), \quad T_{2}(x, y, t)=(-x, y,-t) \quad \text { and } \quad T_{3}(x, y, t)=(x,-y,-t) .
$$

Proof. By a rotation the vector field $X_{(a, g, c, e)}$ is transformed into $X_{(0, \tilde{g}, \tilde{c}, \tilde{e})}$ for some parameter value $(0, \tilde{g}, \tilde{c}, \tilde{e})$. Indeed the rotation $\theta \mapsto \theta-\theta_{0}$ is in cartesian coordinates defined by the matrix

$$
M\left(\theta_{0}\right) \equiv\left(\begin{array}{cc}
\cos \theta_{0}-\sin \theta_{0} \\
\sin \theta_{0} & \cos \theta_{0}
\end{array}\right),
$$

and transforms the vector field $X_{(a, g, c, e)}$ into the vector field $X_{K\left(\theta_{0}\right)(a, g, c, e)}$, where the linear map $K\left(\theta_{0}\right)$ is given by

$$
K\left(\theta_{0}\right)=\left(\begin{array}{cccc}
\left(3 \cos ^{2} \theta_{0}-2\right) \cos \theta_{0} & \left(1-3 \cos ^{2} \theta_{0}\right) \sin \theta_{0} & \cos \theta_{0} \sin ^{2} \theta_{0} & \cos ^{2} \theta_{0} \sin \theta_{0} \\
\left(3 \cos ^{2} \theta_{0}-1\right) \sin \theta_{0} & -2 \cos \theta_{0} & -\sin \theta_{0} \cos ^{2} \theta_{0} \cos \theta_{0} \sin ^{2} \theta_{0} \\
-\cos \theta_{0} \sin ^{2} \theta_{0} & -\cos ^{2} \theta_{0} \sin \theta_{0} & -\cos ^{3} \theta_{0} / 3 & -\sin ^{3} \theta_{0} / 3 \\
-3 \sin \theta_{0} \cos ^{2} \theta_{0} & 3 \cos \theta_{0} \sin ^{2} \theta_{0} & -\sin ^{3} \theta_{0} & \cos ^{3} \theta_{0}
\end{array}\right)
$$

with $\operatorname{det} K\left(\theta_{0}\right)=-1 / 3 \neq 0$. Furthermore since $A(0)=-A(\pi)=a$, there exists an angle $\theta_{0} \in[0, \pi]$ and $1 \leq n \leq 3$ such that $A^{(i)}\left(\theta_{0}\right)=0$, for all $i \leq n-1$ and $A^{(n)}\left(\theta_{0}\right) \neq 0$.

By $T_{1}$ (resp. $T_{2}$ and $\left.T_{3}\right)$ the vector field $X_{(a, g, c, e)}$ is transformed into $X_{(-a,-g,-c,-e)}$ (resp. $-X_{(a,-g, c,-e)}$ and $\left.-X_{(-a, g,-c, e)}\right)$.

In particular we can fix the sign of the first non-vanishing derivative of $A$ at $\theta=0$ since

$$
A^{(n)}\left(\theta_{0}+\pi\right)=-A^{(n)}\left(\theta_{0}\right) \text {. }
$$

As it is convenient in cases that $A$ has at most two zeroes in $[0, \pi)$ we shall assume that

$$
A^{(n)}\left(\theta_{0}\right)>0
$$

in cases that $A$ has three zeroes in $[0, \pi)$ we shall assume that

$$
A^{(n)}\left(\theta_{0}\right)<0 .
$$




\subsection{Classification of the singularities}

For a given $\theta^{*}$ we define the ray $\theta=\theta^{*}$ by the set $\left\{\left(r, \theta^{*}\right): r \geq 0\right\}$. Then for $B\left(\theta^{*}\right)=-2$ system (9) has exactly one singularity on the ray $\mathcal{R}^{*}$ different from the center at the origin, and its radius is 1 . For $B\left(\theta^{*}\right)<-2$ system (9) has two singularities on the ray $\mathcal{R}^{*}$ with radius $r_{ \pm}^{*}$. For later use we describe the behavior of $r_{ \pm}^{*}$ with respect to $B=B\left(\theta^{*}\right)$ in Lemma 3, and illustrate its graph in Figure 3 .

Lemma 3. Let $B<-2$ and let $r_{ \pm}$be the radii defined as

$$
r_{ \pm}=\frac{-B \pm \sqrt{B^{2}-4}}{2} .
$$

The graphs of $r_{ \pm}$exhibit the following behavior (see Figure 3):

(1) The graph of $r_{+}$is concave and decreasing to 1 as $B$ increases to -2 .

(2) The graph of $r_{-}$is convex and increasing to 1 as $B$ increases to -2 .

(3) $r_{+}+r_{-}=-B$ and $r_{+} \cdot r_{-}=1$.

(4) If $B_{1}<B_{2}<-2$, then $r_{-}\left(B_{1}\right)<r_{-}\left(B_{2}\right)<r_{+}\left(B_{2}\right)<r_{+}\left(B_{1}\right)$.

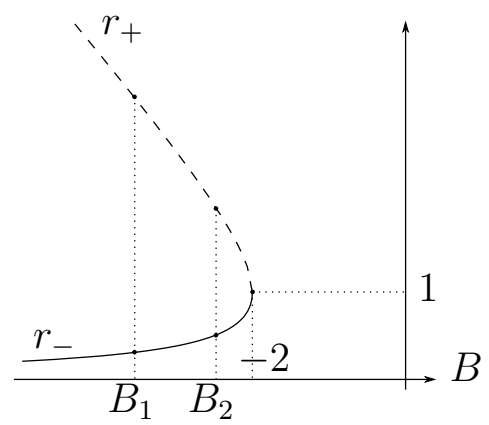

Fig. 3. Graphs of $r_{+}$and $r_{-}$as functions of $B$, drawn in dashed line and continuous line respectively.

Since $A$ and $B$ are cubic homogeneous polynomials in $(\cos \theta, \sin \theta)$ it follows that

$$
A(\theta+\pi)=-A(\theta) \text { and } B(\theta+\pi)=-B(\theta),
$$

and the same property holds for their derivatives with respect to $\theta$. As a consequence there are at most three angles $0 \leq \theta_{0}, \theta_{1}, \theta_{2} \leq 2 \pi$ satisfying $A\left(\theta_{i}\right)=0$ such that the equation $r^{2}+B\left(\theta_{i}\right) r+1=0$ has at least one positive solution $r, i=0,1,2$. As a conclusion, system (9) has at most 7 singularities, one singularity fixed at the origin and the other singularities laying on the rays $\theta=\theta_{i}$ for $i=0,1,2$.

From the relation (22) all zeroes of $A$ can be derived from the zeroes in $[0, \pi)$, by translating these zeroes over integer multiples of $\pi$. Furthermore the graph of $A$ on $[\pi, 2 \pi)$ is found by reflecting the graph of $A$ on $[0, \pi)$ about the $\theta$-axis and translating it over a distance of $\pi$. If $A$ vanishes along the ray $\theta=0$, then the horizontal axis can carry singularities; singularities are present on the positive horizontal axis, i.e. on the ray $\theta=0$, only if $B(0) \leq-2$. If $B(0)>-2$, then there are no singularities present on the positive horizontal axis. By $(22)$ if $B(0) \geq 2$, then singularities are present on the negative horizontal axis, and if $|B(0)|<2$, no singularities occur on the horizontal axis at all. Analogously if $A$ vanishes along the ray $\theta=\theta^{*}$, there are singularities present on $\mathcal{R}^{*} \equiv\left\{\theta=\theta^{*}\right\}$ (respectively $\mathcal{R}^{*} \equiv\left\{\theta=\theta^{*}+\pi\right\}$ ) if $B\left(\theta^{*}\right) \leq-2$ (respectively if $B\left(\theta^{*}\right) \geq 2$ ). If $\left|B\left(\theta^{*}\right)\right|<2$, then there are no singularities neither on $\theta=\theta^{*}$ nor on $\theta=\theta^{*}+\pi$.

To determine the nature of each singularity $\left(x^{*}, y^{*}\right)=\left(r^{*} \cos \theta^{*}, r^{*} \sin \theta^{*}\right)$ of $(9)$, we analyze its Jacobian $J$ at $\left(x^{*}, y^{*}\right)$, expressed in cartesian coordinates $(x, y)$. The determinant of $J$ at the singularity $\left(x^{*}, y^{*}\right)$ is given by

$$
\operatorname{det} J\left(x^{*}, y^{*}\right)=\operatorname{det}\left(\begin{array}{cc}
2 r^{*} A\left(\theta^{*}\right) & \left(r^{*}\right)^{2} A^{\prime}\left(\theta^{*}\right) \\
B\left(\theta^{*}\right)+2 r^{*} & r^{*} B^{\prime}\left(\theta^{*}\right)
\end{array}\right)=r^{*} A^{\prime}\left(\theta^{*}\right)\left(1-\left(r^{*}\right)^{2}\right)
$$


and its trace by $\operatorname{tr}\left(J\left(x^{*}, y^{*}\right)\right)=0$.

If $\operatorname{det} J\left(x^{*}, y^{*}\right) \neq 0$, then the singularity $\left(x^{*}, y^{*}\right)$ is elementary. In particular since the system is Hamiltonian, if $\operatorname{det} J\left(x^{*}, y^{*}\right)>0$ (respectively $<0$ ), then the singularity is a center (respectively saddle).

If $\operatorname{det} J\left(x^{*}, y^{*}\right)=0$, then the singularity $\left(x^{*}, y^{*}\right)$ is degenerate; it is said to be non-elementary if both eigenvalues of $J\left(x^{*}, y^{*}\right)$ vanish. Using a local classification theorem for nilpotent singularities (see e.g., [Dumortier et al., 2006]), the topological type of the degenerate singularities can be determined in cases $B\left(\theta^{*}\right)<-2$ and $B\left(\theta^{*}\right)=-2$ with $A^{\prime}\left(\theta^{*}\right) \neq 0$. However by a detailed analysis of the asymptotics of the system, we can describe the local behavior of all degenerate singularities more precisely (see Proposition 1 ).

More precisely elementary singularities lay on rays $\theta=\theta^{*}$, for which $A\left(\theta^{*}\right)=0$ and $B\left(\theta^{*}\right)<-2$. In this case if $A^{\prime}\left(\theta^{*}\right)<0$ (resp. $>0$ ), the singularity determined by $\left(r_{-}^{*}, \theta^{*}\right)$ (resp. $\left(r_{+}^{*}, \theta^{*}\right)$ ) is a saddle point and the singularity determined by $\left(r_{+}^{*}, \theta^{*}\right)$ (resp. $\left.\left(r_{-}^{*}, \theta^{*}\right)\right)$ is a center point, i.e., if $A^{\prime}\left(\theta^{*}\right)<0$ (resp. $>0$ ), then on the ray $\theta=\theta^{*}$ the singularity with smaller radius is a saddle (resp. center), while the singularity with bigger radius is a center (resp. saddle).

In Proposition 1 of section 4.3 we summarize all possible types of the Hamiltonian singularities in terms of the multiplicity of $A$ on the ray $\theta=\theta^{*}$, and the sign of $A(\theta)$ for $\theta \searrow \theta^{*}$. In fact given $\theta^{*}$ such that $A\left(\theta^{*}\right)=0$, i.e., on the ray $\theta=\theta^{*}$ singularities can be present, the proposition gives the number, the relative position and the type of the singularities on the ray $\theta=\theta^{*}$ in terms of the first non-vanishing derivative $A$ at $\theta^{*}$.

The proof of this proposition and the principal result strongly relies on the relation between the trigonometric polynomials $A$ and $B$, as described in the following lemma.

Lemma 4. Let $A$ and $B$ be the cubic trigonometric polynomials defined in (12). Then for all $k \geq 1$ and for all $\theta \in \mathbb{R}$ we have that $B^{(k)}(\theta)=-3 A^{(k-1)}(\theta)$.

Lemma 5. Let $A, B$ be the cubic trigonometric polynomials as defined in (12) and $\theta^{*} \in \mathbb{R}$. Then the following statements are equivalent:

(1) $A\left(\theta^{*}\right)=A^{\prime}\left(\theta^{*}\right)=A^{\prime \prime}\left(\theta^{*}\right)=A^{(3)}\left(\theta^{*}\right)=0$.

(2) $a=c=e=g=0$.

In particular if one of the above statements is satisfied, then (9) has a global center at the origin linearly equivalent to $\{\dot{x}=-y, \quad \dot{y}=x\}$.

In Figure 4 the relation between the graphs of $A$ and $B$ is sketched in case $A$ vanishes along the horizontal axis according to the assumptions made in (18), (19) and (20). By Lemma 5, we can distinguish between four non-trivial cases:

- Case 1 corresponds to $A(0)=A^{\prime}(0)=A^{\prime \prime}(0)=0, A^{\prime \prime \prime}(0) \neq 0$;

- Case 2 corresponds to $A(0)=A^{\prime}(0)=0, A^{\prime \prime}(0) \neq 0$;

- Cases 3 and 4 correspond to $A(0)=0, A^{\prime}(0) \neq 0$.

Case 3 is distinguished from Case 4 by their number of zeroes in $(0, \pi)$ : in Case $3 A$ has no zeroes in $(0, \pi)$, while in Case $4, A$ has 2 zeroes in $(0, \pi)$.

Due to the number of zeroes of $A$ in $[0,2 \pi]$, multiplicity taken into account, we will call Cases $1,2,3$ and 4 respectively by 'One triple ray', 'One double ray - one simple ray', 'One simple ray - two complex rays', and 'Three simple rays'.

Next proposition describes the local phase portrait of the Hamiltonian system (9) in a neighborhood of the rays $\theta=\theta^{*}$ along which $A$ vanishes.

Proposition 1. Let $A$ and $B$ be the trigonometric polynomials of degree 3 defined in (12), and suppose that for $1 \leq n \leq 3$,

$$
A^{(i)}\left(\theta^{*}\right)=0, \text { for all } 0 \leq i \leq n-1
$$

and $\theta^{*} \in \mathbb{R}$ such that

$$
n ! \gamma \equiv A^{(n)}\left(\theta^{*}\right) \neq 0 .
$$




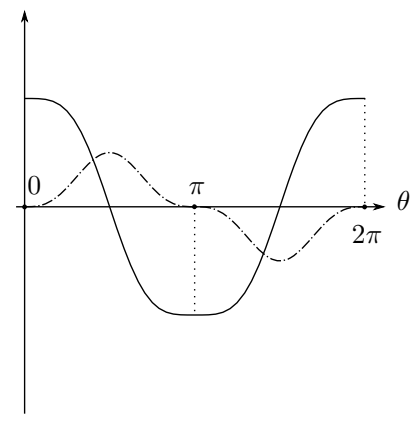

Case 1: One triple ray

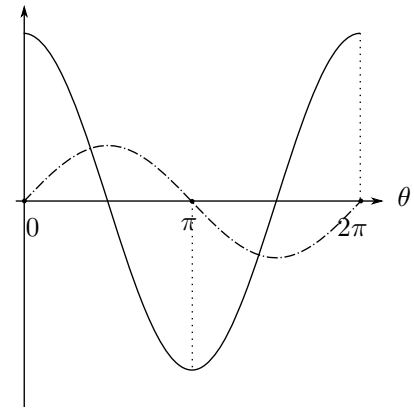

Case 3: One simple ray - two complex rays

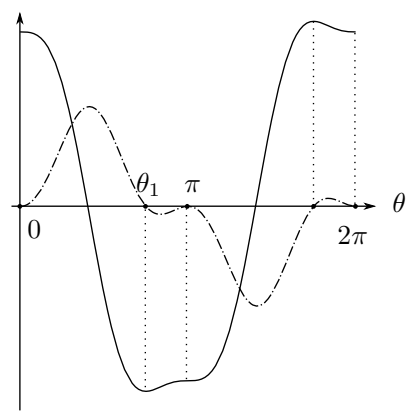

Case 2: One double ray- one simple ray

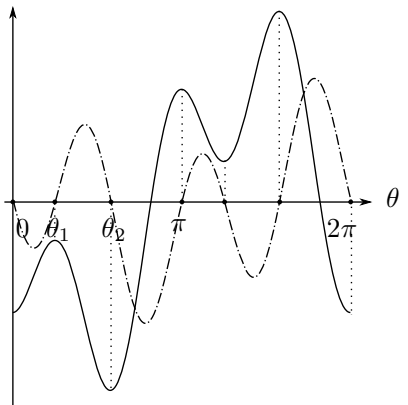

Case 4: Three simple rays

Fig. 4. Graphical analysis of the map $B$ in relation with the map $A$ as functions of $\theta$ in $[0,2 \pi]$. The map $B$ is drawn in continuous line and $A$ is drawn in dashed line. The parameter values are: Case $1, a=0, e=2, g=1, c=0$; Case 2, $a=0, c=1, g=1, e=2$; Case $3, a=0, c=0, g=1, e=3$ and Case $4, a=0, e=-13 / 2, g=13 / 4, c=39 / 4$ or $\alpha=1, \beta=-1 / 4, \lambda=13$.

\section{Then the origin is a center. Furthermore}

(1) If $B\left(\theta^{*}\right)>-2$, then there are no additional singular points on the ray $\theta=\theta^{*}$. The local phase portrait of (9) along the ray $\theta=\theta^{*}$ is given in Figure 5.

\begin{tabular}{|c|c|c|c|c|c|}
\hline \multicolumn{6}{|c|}{$\left|B\left(\theta^{*}\right)\right|<2$} \\
\hline \multicolumn{3}{|c|}{$\gamma<0$} & \multicolumn{3}{|c|}{$\overline{\gamma \gamma>0}$} \\
\hline$n=1$ & $n=2$ & $n=3$ & $n=1$ & $n=2$ & $n=3$ \\
\hline \multicolumn{6}{|c|}{$\left|B\left(\theta^{*}\right)\right|=2$} \\
\hline (C) & (8) & (3) & (B) & & (3) \\
\hline \multicolumn{6}{|c|}{$\mid \overline{\left|B\left(\theta^{*}\right)\right|>2}$} \\
\hline (2) & (क) & (3) & (क) (e) & (क) & \\
\hline
\end{tabular}

Fig. 5. Classification of the phase portraits of (9) in a neighborhood of the ray $\theta=\theta^{*}$; see Proposition 1. 
(2) If $B\left(\theta^{*}\right)=-2$, then there is one additional singular point on the ray $\theta=\theta^{*}$ for which the radius $r=1$. Depending on the first non-vanishing higher order derivative $A^{(n)}\left(\theta^{*}\right)$ and its sign, the local phase portrait of (9) along the ray $\theta=\theta^{*}$ is given in Figure 5.

(3) If $B\left(\theta^{*}\right)<-2$, then there are two additional singularities on the ray $\theta=\theta^{*}$ for which the radii are $0<r_{-}<1<r_{+}$with expressions given in (14). Depending on the first non-vanishing higher order derivative $A^{(n)}\left(\theta^{*}\right)$ and its sign, the local phase portrait of (9) along the ray $\theta=\theta^{*}$ is given by Figure 5 .

Proof. It suffices to analyze the behavior of the singularities on the ray $\mathcal{R}^{*} \equiv\left\{\theta=\theta^{*}\right\}$ for which $B\left(\theta^{*}\right) \leq-2$. Every solution $(r, \theta)$ of (11) sufficiently close to $\left(r^{*}, \theta^{*}\right)$ with $1+r^{*} B\left(\theta^{*}\right)+\left(r^{*}\right)^{2}=0$ can be parameterized by $\theta$, say $r=r(\theta)$, where $r(\theta)$ is solution of

$$
\left(1+r B(\theta)+r^{2}\right) \frac{\mathrm{d} r}{\mathrm{~d} \theta}=r^{2} A(\theta) .
$$

By Lemma 4, relations (24) and (25) we can write for $\theta \rightarrow \theta^{*}$

$$
\begin{aligned}
& A(\theta)=\gamma\left(\theta-\theta^{*}\right)^{n}+O\left(\left(\theta-\theta^{*}\right)^{n+1}\right), \\
& B(\theta)=B\left(\theta^{*}\right)-\frac{3 \gamma}{n+1}\left(\theta-\theta^{*}\right)^{n+1}+O\left(\left(\theta-\theta^{*}\right)^{n+2}\right) .
\end{aligned}
$$

Since $r^{*} \neq 0$ on a sufficiently small compact neighborhood of $\left(r^{*}, \theta^{*}\right)$ the asymptotics of $(26)$ is as follows

$$
\frac{1}{r^{2}}\left(1+r B\left(\theta^{*}\right)+r^{2}\right) \frac{\mathrm{d} r}{\mathrm{~d} \theta}=\gamma\left(\theta-\theta^{*}\right)^{n}+O\left(\left(\theta-\theta^{*}\right)^{n+1}\right), \quad \theta \rightarrow \theta^{*} .
$$

Now the result follows by applying Lemma 6 on (27).

Lemma 6. Let $k, n \geq 1$. Consider the planar differential vector field $X$ with a singularity at $\left(x_{0}, y_{0}\right)$, such that in polar coordinates $(r, \theta)$ the singularity $x_{0}$ corresponds to $\left(r_{0}, \theta_{0}\right)$ and $X$ writes as $\{\dot{r}=F(r, \theta), \dot{\theta}=$ $G(r, \theta)\}$ for some smooth functions $F, G$ with asymptotics

$$
F(r, \theta)=\gamma \cdot\left(\theta-\theta_{0}\right)^{n}+\bar{F}(r, \theta) \quad \text { and } \quad G(r, \theta)=\left(r-r_{0}\right)^{k}+\bar{G}(r, \theta),
$$

where $\gamma \neq 0, \bar{F}(r, \theta)=O\left(\left(\theta-\theta_{0}\right)^{n+1}\right)$ and $\bar{G}(r, \theta)=O\left(\left(r-r_{0}\right)^{k+1}\right)$, for $\theta \rightarrow \theta_{0}, r \rightarrow r_{0}$. Then, the diffeomorphic type of the singularity $\left(x_{0}, y_{0}\right)$ depends on the parity of $k$ and $n$, the ratio $k / n$ and the sign of $\gamma$ as shown in Figure 6. For $k, n$ both odd the singularity is a topological saddle, for $k$ and $n$ of opposite parity the singularity is a cusp, and for $k, n$ both even the phase portrait near the singularity shows that of a parallel flow.

Proof. The system can be written as a scalar differential system $G(r, \theta) \mathrm{d} r=F(r, \theta) \mathrm{d} \theta$. By integration along the path from $\left(r_{0}+\delta, \theta_{0}+\varepsilon\right)$ to $(r, \theta)$ for $|\delta|,|\varepsilon|,\left|r-r_{0}\right|,\left|\theta-\theta_{0}\right|$ small, we get the following asymptotics:

$$
\left(r-r_{0}\right)^{k+1}=\frac{\gamma(k+1)}{n+1}\left(\theta-\theta_{0}\right)^{n+1}+U(r, \theta, \delta, \varepsilon),
$$

with $U(r, \theta, \delta, \varepsilon)=O\left(\left(\theta-\theta_{0}\right)^{n+1}\right)+O\left(\left(r-r_{0}\right)^{k+1}\right)+O\left(\delta^{k}\right)+O\left(\varepsilon^{n}\right)$, for $(r, \theta, \delta, \varepsilon) \rightarrow\left(r_{0}, \theta_{0}, 0,0\right)$. Studying the graph of equation (29) we obtain the topological type of $\left(x_{0}, y_{0}\right)$ corresponding to $\left(r_{0}, \theta_{0}\right)$ as drawn in Figure 6.

Remark 4.1.

(1) By the classification in Lemma 6 we will call the integers $n$ (resp. $k$ ) the multiplicity of the ray $\left\{\theta=\theta_{0}\right\}$, (resp. the multiplicity of the singularity $r=r_{0}$ on this ray). Furthermore we will call $\gamma$ the orientation of the singularity on the ray $\left\{\theta=\theta_{0}\right\}$. 


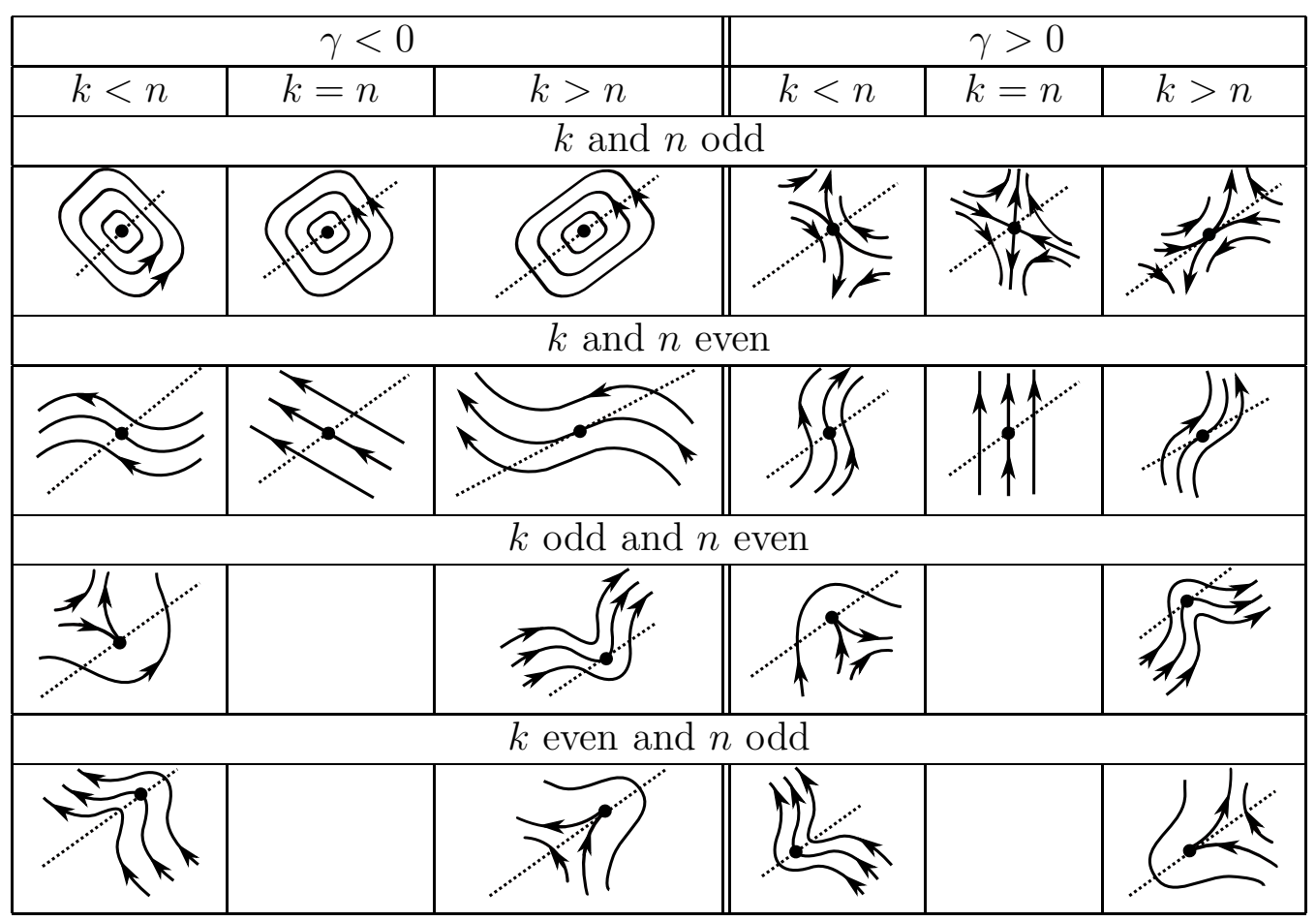

Fig. 6. Classification of the singularity $\left(x_{0}, y_{0}\right)=\left(r_{0} \cos \theta_{0}, r_{0} \sin \theta_{0}\right)$ of $\left\{\dot{r}=\gamma \cdot\left(\theta-\theta_{0}\right)^{n}+\bar{F}(r, \theta), \dot{\theta}=\left(r-r_{0}\right)^{k}+\bar{G}(r, \theta)\right\}$ where $(r, \theta)$ are polar coordinates, see Lemma 6.

(2) From Proposition 1 the Hamiltonian singularities can topologically be summarized to be one of the following three types: center, saddle or cusp. In particular parameters for which $B$ equals -2 for a certain root $\theta^{*}$ of $A$, show locally along the ray $\mathcal{R}^{*}$ a topologically equivalent behavior for fixed sign of $\gamma$. For $n=1$ the singularity different from $(0,0)$ is a nilpotent cusp; for $n=2$ resp. 3, these singularities have zero linear part, and therefore we will call them degenerate cusp of order 1 resp. 2. Analogously parameters for which $B$ is strictly smaller than -2 for a certain root $\theta^{*}$ of $A$ show locally along the ray $\mathcal{R}^{*}$ a topologically equivalent behavior for fixed sign of $\gamma$, in case $n=1$ or 3 . The saddles and centers outside the origin are elementary for $n=1$ while they are nilpotent for $n=3$. For $n=2$ the singularities different from $(0,0)$ are nilpotent cusps.

\subsection{Properties of the Hamiltonian}

In this section we summarize the properties and the relative values of the Hamiltonian $H$ as defined in (10) at the singular points. Observe that the Hamiltonian $H$ depends on the parameter only through $B$. Moreover the radii of the singularities are completely determined by the value of $\mathrm{B}$; as a consequence the value of the Hamiltonian and its properties near a ray $\theta=\theta^{*}$ implicitly depend on the parameter through $B=B\left(\theta^{*}\right)$.

For $r, B \in \mathbb{R}$ we write

$$
\bar{H}_{B}(r)=r^{2}\left(\frac{1}{2}+\frac{1}{3} B r+\frac{1}{4} r^{2}\right) .
$$

Lemma 7 and Figure 7 summarize the analytical interpretation and the graphical properties of $\bar{H}_{B}$. In particular $\bar{H}_{B}(r)\left(\right.$ resp. $\left.\bar{H}_{B}(-r)\right)$ for $r>0$ represents the value of the Hamiltonian at $\left(r \cos \left(\theta^{*}\right), r \sin \left(\theta^{*}\right)\right)$ (resp. $\left(r \cos \left(\theta^{*}+\pi\right), r \sin \left(\theta^{*}+\pi\right)\right)$ if $B=B\left(\theta^{*}\right)$.

Lemma 7. Let $\bar{H}_{B}(r)$ be the function defined in (30), then its graph is drawn in Figure \%. Furthermore

(1) For $(x, y)=(r \cos \theta, r \sin \theta)$, we have $H(x, y)=\bar{H}_{B}(r)$, where $H(x, y)$ is the Hamiltonian defined in (10) and $B=B(\theta)$ is the trigonometric polynomial defined in (12). 


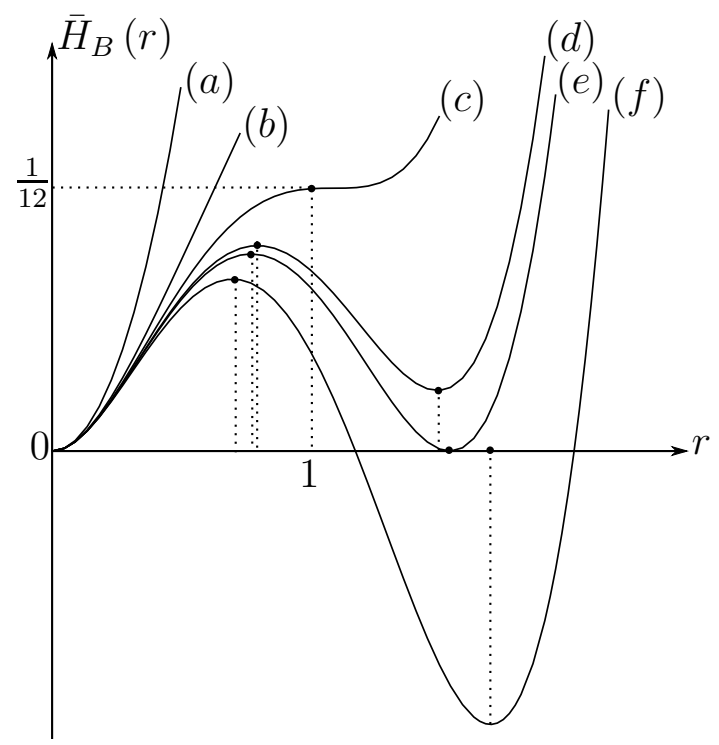

Fig. 7. Graph of $\bar{H}_{B}(r)$ for characteristic values of $B$; see Lemma 9. The corresponding values of $B$ are: $(a)-\sqrt{3}<B$, (b) $-2<B \leq-\sqrt{3},(c) B=-2,(d)-3 \sqrt{2} / 2<B<-2,(e) B=-3 \sqrt{2} / 2$ and $(f) B<-3 \sqrt{2} / 2$.

(2) If $B=-2$ then $\bar{H}_{-2}(1)=\frac{1}{12}$.

(3) For $B<-2$ we define the functions $h_{ \pm}:(-\infty,-2) \rightarrow \mathbb{R}$ by

$$
h_{ \pm}(B) \equiv \bar{H}_{B}\left(r_{ \pm}\right)=-\frac{1}{48}\left(-2+B^{2} \mp B \sqrt{B^{2}-4}\right)\left(-6+B^{2} \mp B \sqrt{B^{2}-4}\right),
$$

i.e., the Hamiltonian value at the singularities $\left(x^{*}, y^{*}\right)=\left(r_{ \pm} \cos \theta^{*}, r_{ \pm} \sin \theta^{*}\right)$ on the ray $\theta=\theta^{*}$ for which $B=B\left(\theta^{*}\right)$ and $r_{ \pm}$is given by $(21)$.

(4) The graph of the functions $h_{ \pm}$are drawn in Figure 8. In particular these functions satisfy the following properties:

(a) The function $h_{+}$is increasing and concave with respect to the variable $B<-2$, i.e., $h_{+}^{\prime}(B)>0$ and $h_{+}^{\prime \prime}(B)<0$ if $B<-2$.

(b) The function $h_{-}$is increasing and convex with respect to the variable $B<-2$, i.e., $h_{-}^{\prime}(B)>0$ and $h_{-}^{\prime \prime}(B)>0$ if $B<-2$.

(c) $\lim _{B \rightarrow-\infty} h_{+}(B)=-\infty$ and $\lim _{B \rightarrow-2} h_{+}(B)=1 / 12$.

(d) $\lim _{B \rightarrow-\infty} h_{-}(B)=0$ and $\lim _{B \rightarrow-2} h_{-}(B)=1 / 12$.

(e) $h_{-}(B)>h_{+}(B)$ if $B<-2$.

(f) $h_{-}(B)>0$ if $B<-2$ and $\begin{cases}h_{+}(B)<0 & \text { if } B<-3 \sqrt{2} / 2, \\ h_{+}(B)=0 & \text { if } B=-3 \sqrt{2} / 2, \\ h_{+}(B)>0 & \text { if }-3 \sqrt{2} / 2<B<-2 .\end{cases}$

Proof. The expression in (31) is obtained using (10) and the fact that $r_{ \pm}^{2}=-1-B r_{ \pm}$. Next by substituting the expressions for $r_{ \pm}$into $h_{ \pm}(B)$, one finds the expression in (31):

$$
h_{ \pm}(B)=-\frac{1}{12}\left(1+B r_{ \pm}\right)\left(3+B r_{ \pm}\right)=-\frac{1}{48} \underbrace{\left(-2+B^{2} \mp B \sqrt{B^{2}-4}\right)}_{>0 \text { if } B<-2}\left(-6+B^{2} \mp B \sqrt{B^{2}-4}\right) .
$$

From this expression the inequalities in statement $(4)(f)$ are easily derived. In particular statement $(4)(e)$ then follows from

$$
h_{-}(B)-h_{+}(B)=-B\left(B^{2}-4\right)^{\frac{3}{2}} / 12 .
$$

For the properties on the derivatives with respect to $B$ in statement $(4)(a)$ and $(b)$ we notice that 


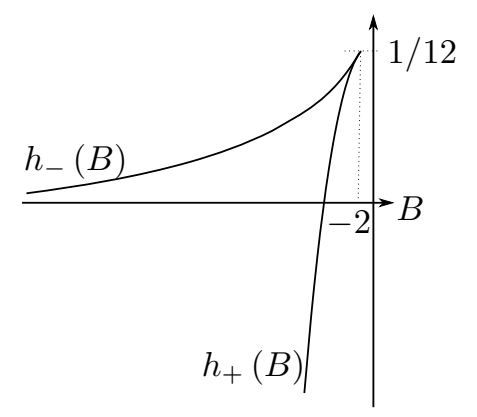

Fig. 8. Graphs of $h_{+}$and $h_{-}$with respect to $B$; see Lemma 7 .

$$
\begin{aligned}
12 \sqrt{B^{2}-4} \cdot h_{ \pm}^{\prime}(B) & =\left(-2+B^{2} \mp B \sqrt{B^{2}-4}\right)\left(-6+B^{2} \mp B \sqrt{B^{2}-4}\right) \text { and } 2 \sqrt{B^{2}-4} \cdot h_{ \pm}^{\prime \prime}(B)= \\
\pm B^{3}-B^{2} \sqrt{B^{2}-4} \mp 3 B & +\sqrt{B^{2}-4} .
\end{aligned}
$$

Lemma 8. Let $\bar{H}_{B}(r)$ be defined in (30). Then the following statements hold

(1) The radial direction of the flow on a fixed ray $\theta=\theta_{1}$ can be read from the graph of $\bar{H}_{B}$, where $B=B(\theta)$ :

$$
\frac{\partial}{\partial r} \bar{H}_{B}(r)=r \dot{\theta}
$$

(2) Let $\left(x^{*}, y^{*}\right) \in \mathbb{R}^{2}$ represented in polar coordinates by $\left(r^{*}, \theta^{*}\right), r^{*}>0$, where $x^{*}=r^{*} \cos \theta^{*}, y^{*}=r^{*} \sin \theta^{*}$. Then $\left(x^{*}, y^{*}\right)$ is a singularity of (9) if and only if $\left(r^{*}, \theta^{*}\right)$ is a solution of

$$
\frac{\partial}{\partial r} \bar{H}_{B\left(\theta^{*}\right)}\left(r^{*}\right)=0 \text { and } \frac{\partial}{\partial \theta} \bar{H}_{B\left(\theta^{*}\right)}\left(r^{*}\right)=0,
$$

as defined in (30).

Proof. Straight-forward derivation of (30) implies the equality in (32). Furthermore it follows that the equations in (33) are equivalent to (13) with $A=\left.\bar{A}\right|_{b=-2 g, f=-2 a}, B=\left.\bar{B}\right|_{b=-2 g, f=-2 a}$.

From this lemma it follows that the critical points of $H_{B}$ with respect to $r$ correspond exactly to the singularities of the Hamiltonian vector field. Next lemma further analyzes the graph of $H_{B}$ with respect to the critical points of the Hamiltonian along a given ray $\theta=\theta^{*}$, for which $A\left(\theta^{*}\right)=0$ and $B=B\left(\theta^{*}\right)$; see Figure 7.

Lemma 9. Consider the function $\bar{H}_{B}: \mathbb{R} \rightarrow \mathbb{R}$ defined by (30) for $B$ in $\mathbb{R}$.

(1) If $B>-2$, then $\bar{H}_{B}$ has 1 critical point over $\mathbb{R}, r=0$, it is a global minimum.

(2) If $B=-2$, then $\bar{H}_{B}$ has 2 critical points over $\mathbb{R}$, a global minimum at $r=0$ and a point of inflection at $r=1$.

(3) If $B<-2$, then $\bar{H}_{B}$ has 3 critical points over $\mathbb{R}, \bar{H}_{B}$ has a local minimum at $r=0$ and at $r=r_{+}$, and a local maximum at $r=r_{-}$, where $r_{ \pm}$are defined in (21). In this case we can distinguish between the following 3 cases:

(a) $0=\bar{H}_{B}(0)<\bar{H}_{B}\left(r_{+}\right)<\bar{H}_{B}\left(r_{-}\right)$, for $-3 \sqrt{2} / 2<B<-2$,

(b) $0=\bar{H}_{B}(0)=\bar{H}_{B}\left(r_{+}\right)<\bar{H}_{B}\left(r_{-}\right)$, for $B=-3 \sqrt{2} / 2$ and

(c) $\bar{H}_{B}\left(r_{+}\right)<0=\bar{H}_{B}(0)<\bar{H}_{B}\left(r_{-}\right)$, for $B<-3 \sqrt{2} / 2$.

(4) Let $B_{1}, B_{2} \in \mathbb{R}$ with $B_{1} \geq B_{2}$, then for $r \in \mathbb{R}$ we have $\bar{H}_{B_{1}}(r)-\bar{H}_{B_{2}}(r)=\frac{1}{3} r^{3}\left(B_{1}-B_{2}\right) \geq 0$. As a consequence the graphs of $\bar{H}_{B_{1}}$ and $\bar{H}_{B_{2}}$ only coincide at $(0,0)$ if $B_{1} \neq B_{2}$. In particular the graph of $\bar{H}_{B_{2}}$ lies below the graph of $\bar{H}_{B_{1}}$. 
Proof. Straight-forward calculations show that for all $B \in \mathbb{R}$ we get

$$
\frac{\partial^{2} \bar{H}_{B}}{\partial r^{2}}(0)=1>0 \text {. }
$$

For $B>-2$, there are no other critical points than $r=0$, but the graph of $\bar{H}_{B}$ is convex or convexconcave-convex depending whether $B>-\sqrt{3}$ or $-2 \leq B<-\sqrt{3}$ (see Figure 7). For $B=-2$ we have

$$
\frac{\partial^{2} \bar{H}_{B}}{\partial r^{2}}(1)=0 \text { and } \frac{\partial^{3} \bar{H}_{B}}{\partial r^{3}}(1)=2 .
$$

For $B<-2$ one finds that

$$
\bar{H}_{B}^{\prime \prime}\left(r_{-}\right)=\frac{B^{2}+B \sqrt{B^{2}-4}-4}{2}<0 \quad \text { and } \quad \bar{H}_{B}^{\prime \prime}\left(r_{+}\right)=\frac{B^{2}-B \sqrt{B^{2}-4}-4}{2}>0 .
$$

Next two lemma's provide a criterion to decide whether a connection between different singularities is possible.

Lemma 10. Let $\left(x_{1}^{*}, y_{1}^{*}\right)$ and $\left(x_{2}^{*}, y_{2}^{*}\right)$ be two singularities of $(2)$ that are not of center type. Then there does not exist a connection between $\left(x_{1}^{*}, y_{1}^{*}\right)$ and $\left(x_{2}^{*}, y_{2}^{*}\right)$ if

(1) $H\left(x_{1}^{*}, y_{1}^{*}\right) \neq H\left(x_{2}^{*}, y_{2}^{*}\right)$, or if

(2) $\left(x_{1}^{*}\right)^{2}+\left(y_{1}^{*}\right)^{2}=1 \neq\left(x_{2}^{*}\right)^{2}+\left(y_{2}^{*}\right)^{2}$.

Lemma 11. Assume that $\left(S_{\lambda}\right)_{\lambda \in \mathbb{R}}$ is a 1 - parameter subfamily of (2) such that for all $\lambda, \theta \in \mathbb{R}$ we have $A(\theta)=\lambda \bar{A}(\theta)$ and $B(\theta)=\lambda \bar{B}(\theta)$ for some trigonometric polynomials $\bar{A}$ and $\bar{B}$ in $\theta$, that are independent of $\lambda$. Suppose that $\theta_{1}, \theta_{2} \in[0,2 \pi)$ with $\bar{A}_{i} \equiv \bar{A}\left(\theta_{i}\right)=0$ and $\bar{B}_{1} \equiv \bar{B}\left(\theta_{1}\right)<\bar{B}_{2} \equiv \bar{B}\left(\theta_{2}\right)<-2$. Let $s_{ \pm}^{i}(\lambda), i=1,2$ be the singularities of $S_{\lambda}$ for $\lambda>-2 / \bar{B}_{2}$ with polar coordinates $\left(r_{ \pm}\left(\lambda \bar{B}_{i}\right), \theta_{i}\right), i=1,2$. Then

(1) there is no connection between $s_{-}^{1}(\lambda)$ and $s_{-}^{2}(\lambda)$ neither between $s_{+}^{1}(\lambda)$ and $s_{-}^{2}(\lambda)$.

(2) If $s_{-}^{1}(\lambda)$ and/or $s_{+}^{2}(\lambda)$ are centers, then there is no connection between $s_{-}^{1}(\lambda)$ and $s_{+}^{2}(\lambda)$;

(3) if for $\lambda>-2 / \bar{B}_{2}$ neither of the singularities $s_{-}^{1}(\lambda)$ nor $s_{+}^{2}(\lambda)$ are centers, then there exists a unique $\lambda^{*}>-2 / \bar{B}_{2}$ such that there exists a connection between $s_{-}^{1}\left(\lambda^{*}\right)$ and $s_{+}^{2}\left(\lambda^{*}\right) ;$ in particular for $-2 / \bar{B}_{2}<$ $\lambda<\lambda^{*}$, we have $h_{-}\left(\lambda \bar{B}_{1}\right)<h_{+}\left(\lambda \bar{B}_{2}\right)$,

$$
h_{-}\left(\lambda^{*} \bar{B}_{1}\right)=h_{+}\left(\lambda^{*} \bar{B}_{2}\right)
$$

and for $\lambda>\lambda^{*}$ we have $h_{-}\left(\lambda \bar{B}_{1}\right)>h_{+}\left(\lambda \bar{B}_{2}\right)$. Furthermore for all $\lambda \neq \lambda^{*}$ there is no connection between the singularities $s_{-}^{1}(\lambda)$ and $s_{+}^{2}(\lambda)$. The bifurcation parameter $\lambda^{*}$ is implicitly defined by (34), and the bifurcation of the relative Hamiltonian values at the singularities is shown in Figure 9.

Proof. This proof exploits the properties of $H$ described in Lemma 7. Statements (1) and (2) follow from Lemma $7(4)(a)$ and (4)(b). Statement (3) follows from statements (4)(b) and (4)(e) of Lemma 7. The existence of $\lambda^{*}$ defined in (34) follows from statements (4)(c) and (4) (d) of Lemma 7. Indeed we have $B_{2}=\lambda \bar{B}_{2} \rightarrow-\infty$, for $\lambda \rightarrow+\infty$ (since $\bar{B}_{2}$ is fixed in $\mathbb{R}^{-}$), hence by the Intermediate Value Theorem there exists $\lambda_{1}$ such that $h_{+}\left(\lambda_{1} \bar{B}_{2}\right)<0<h_{-}\left(\lambda_{1} \bar{B}_{1}\right)$. On the other hand for $\lambda_{2}=-2 / \bar{B}_{2}$ we have

$$
\lambda_{2} \bar{B}_{1}<\lambda_{2} \bar{B}_{2}=-2 \text { and so } h_{-}\left(\lambda_{2} \bar{B}_{1}\right)<\frac{1}{12}=h_{+}\left(\lambda_{2} \bar{B}_{2}\right) .
$$

Hence there exists $\lambda^{*} \in\left(\lambda_{2}, \lambda_{1}\right)$ such that $h_{-}\left(\lambda^{*} \bar{B}_{1}\right)=h_{+}\left(\lambda^{*} \bar{B}_{2}\right)$. Suppose that $\lambda^{* *}>\lambda^{*}$ also has property (34); then by Rolle's Theorem applied to the smooth function $P$, defined by $P(\lambda)=h_{-}\left(\lambda \bar{B}_{1}\right)-h_{+}\left(\lambda \bar{B}_{2}\right)$, it follows that $h_{-}^{\prime}\left(c \bar{B}_{1}\right)=h_{+}^{\prime}\left(c \bar{B}_{2}\right)$ for some $c \in\left(\lambda^{*}, \lambda^{* *}\right)$; this is in contradiction with the convexity (resp. concavity) property of $h_{-}$(resp. $h_{+}$), implying that

$$
h_{+}^{\prime}\left(c \bar{B}_{2}\right)>h_{+}^{\prime}(-2)=h_{-}^{\prime}(-2)>h_{-}^{\prime}\left(c \bar{B}_{1}\right) .
$$

Using a continuity argument the claim on the connections follows. 


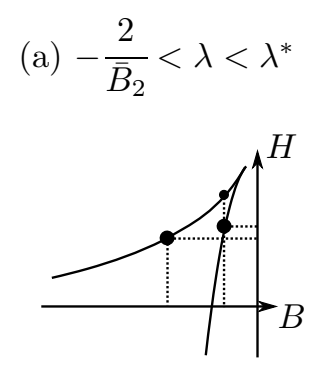

(b) $\lambda=\lambda^{*}$

(c) $\lambda>\lambda^{*}$
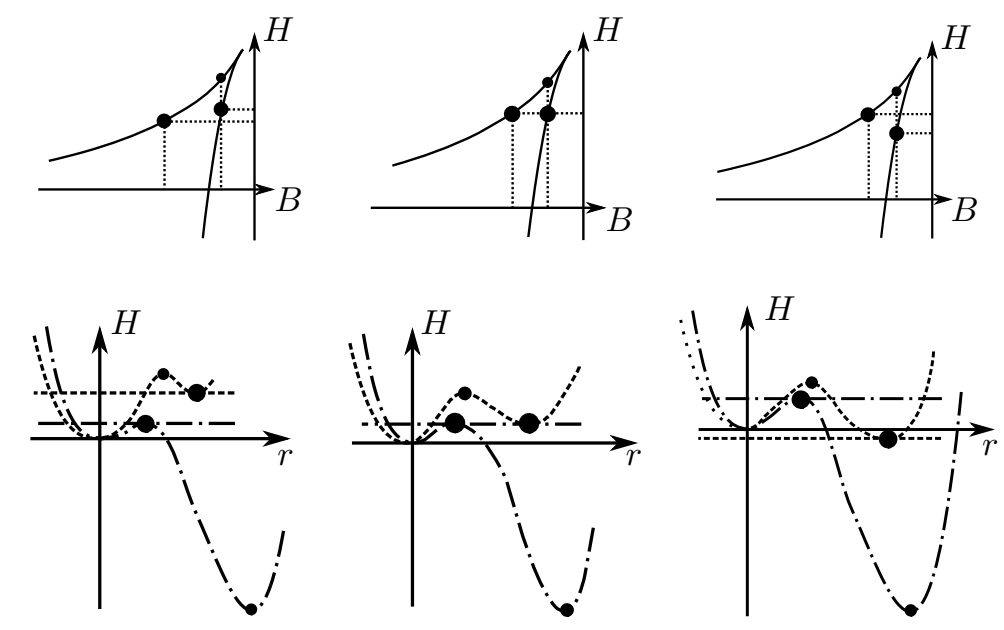

Fig. 9. Bifurcation of the Hamiltonian with respect to $\lambda$, see Lemma 11.

\section{Classification of the Hamiltonian Phase Portraits}

Using Proposition 1 we can classify all Hamiltonian singularities and their local behavior in a systematic way. Now to understand the global behavior, like the relative position of the separatrices and the existence of connections between the singularity points, we make use of the global properties of the Hamiltonian $H$ along a ray carrying singularities (see section 4.4) as well as of a continuity argument (Lemma 11).

In first instance the classification is based on the number of complex zeroes of $A$ in $[0, \pi)$ multiplicity taken into account. For the 3-parameter Hamiltonian family in (2) and (17) the trigonometric function $A$ in (12) reads as:

$$
A(\theta)=\sin \theta\left((e-2 g) \cos ^{2} \theta+c \cos \theta \sin \theta+g \sin ^{2} \theta\right) .
$$

Next the trigonometric function $B$ for this 3-parameter family reads as

$$
B(\theta)=e \cos ^{3} \theta+3 g \cos \theta \sin ^{2} \theta-c \sin ^{3} \theta .
$$

In particular $A$ has always a zero in $[0, \pi)$ at $\theta=0$, and

$$
A^{\prime}(0)=e-2 g, \quad A^{\prime \prime}(0)=2 c .
$$

Therefore by Lemma 5 we only need to treat four cases for which the graph of $A$ can be found in Figure 4; in terms of the parameter $(g, c, e)$ these cases are characterized as follows:

Case 1 or 'One triple ray' $e-2 g=c=0$.

Case 2 or 'One double ray - one simple ray' $e-2 g=0, c \neq 0$.

Case 3 or 'One simple ray - two complex rays' $e-2 g \neq 0$ and $c^{2}-4 g(e-2 g)<0$.

Case 4 or 'Three simple rays' $e-2 g \neq 0$ and $c^{2}-4 g(e-2 g)>0$.

Notice that the case $e-2 g \neq 0$ with $c^{2}-4 g(e-2 g)=0$ is contained in Case 2, i.e., $e-2 g=0, c \neq 0$. Indeed in that case the function $A$ has a double zero at $\theta=\theta_{1} \neq 0$ in $(0, \pi)$; after rotation over angle $\theta_{1}$ and perhaps translation over distance $\pi$ the current system (9) is transformed to one satisfying Case 2 .

The subsections 5.1, 5.2, 5.3 and 5.4 subsequently deal with these four cases. In all of these cases we will use the following notation

$$
0=\theta_{0}<\theta_{1}<\theta_{2}<\pi \quad \text { such that } A\left(\theta_{i}\right)=0, \quad i=0,1,2 \quad \text { (if they exist). }
$$


We denote by $\mathcal{R}_{i}$ the ray

$$
\mathcal{R}_{i}= \begin{cases}\left\{\theta=\theta_{i}, r \geq 0\right\} & \text { if } B\left(\theta_{i}\right) \leq 0, \\ \left\{\theta=\theta_{i}+\pi, r \geq 0\right\} & \text { if } B\left(\theta_{i}\right)>0 .\end{cases}
$$

Furthermore for $i=0,1,2$ we denote

$$
h_{ \pm}^{i}=\bar{H}_{B}\left(r_{ \pm}(B)\right), \text { where } B \equiv-\left|B\left(\theta_{i}\right)\right|,
$$

whenever $\left|B\left(\theta_{i}\right)\right|>2$. If $\left|B\left(\theta_{i}\right)\right|>2$ for $i=0,1,2$, then $h_{ \pm}^{i}$ are the values of the Hamiltonian at the singularities $s_{ \pm}^{i}$ along the ray $\mathcal{R}_{i}$ at a distance $r_{ \pm}$from the origin. If $\left|B\left(\theta_{i}\right)\right|=2$, i.e., when the singularities on $\mathcal{R}_{i}$ collapse, then we will write $h^{i}$ :

$$
h^{i}=\bar{H}_{-2}(1)=\frac{1}{12} .
$$

Lemma 12. Let $B$ be the trigonometric function defined in (12). The origin of (2) is a global center if and only if

$$
\max \{|B(\theta)|: \theta \in \mathbb{R}\}=\max \{|B(\theta)|: \theta \in[0, \pi) \text { such that } A(\theta)=0\}<2 .
$$

Proof. From Lemma 4 it follows that both maxima in (37) coincide. The assertion follows since a linear center of the Hamiltonian system (2) with $\dot{\theta}>1$ for all $(r, \theta)$ is a global center.

\subsection{Case 1: One triple ray}

In this case the functions $A$ and $B$ simplify to

$$
A(\theta)=g \sin ^{3} \theta \quad \text { and } \quad B(\theta)=g \cos \theta\left(2+\sin ^{2} \theta\right) .
$$

For $g=0$ the functions $A$ and $B$ vanish identically. So the phase portrait consists of a global center. For $g>0$ the graph of $A$ is drawn in Figure 4 Case 1. As a consequence one finds

$$
A(0)=A^{\prime}(0)=A^{\prime \prime}(0)=0, A^{\prime \prime \prime}(0)=6 g>0 \quad \text { and } \quad B(0)=e=2 g>0 .
$$

Clearly all singularities are found on $\mathcal{R}_{0}$, which corresponds to the negative horizontal axis. By Proposition 1 the diffeomorphically different phase portraits are determined by the parameter $g$, see Figure 10 .

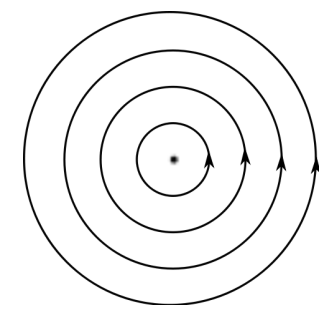

(a) $g<1$

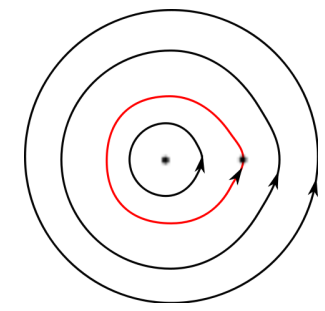

(b) $g=1, h^{0}=\frac{1}{12}$

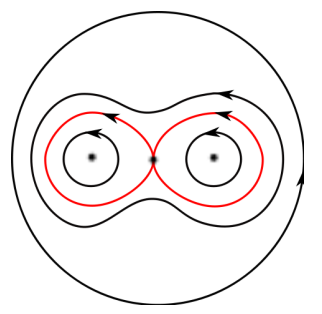

(c) $g>1, h_{+}^{0}<h_{-}^{0}<\frac{1}{12}$

Fig. 10. Phase portraits of (2) in Case $1(e-2 g=c=0)$ (see Theorem 2).

For $g<1$ the phase portrait is a global center. When $g$ grows and reaches $g=1$, a degenerate singularity $s^{0}$ is born on the horizontal axis at $(-1,0)$, and the global center is disturbed by a homoclinic loop. Indeed by Lemma 9 (2), $H$ is strictly increasing with $r \geq 0$, and by continuity with respect to the parameter $g$, the stable and unstable separatrices of the degenerate singularity at $(-1,0)$ connect, enclosing the center at the origin. Moreover $h_{-}^{0}=1 / 12$. When $g$ grows through $g=1$, the cusp singularity splits horizontally into two singularities: a saddle $s_{-}^{0}$ and a center point $s_{+}^{0}$. By the same arguments as before, Lemma 9 and continuity, we know that a figure eight is born; moreover, $h_{+}^{0}<h_{-}^{0}<1 / 12$.

This ends the proof of Theorems 2, 5 and 4 in Case 1. 


\subsection{Case 2: One double ray - one simple ray}

By Lemma 2, we only have to consider parameter values for which $e-2 g=0, c>0, g \geq 0$.

In this case the graph of $A$ looks like the one in Figure 4 Case 2 and the trigonometric functions $A$ and $B$ can be written as

$$
\begin{aligned}
& A(\theta)=c \sin ^{2} \theta(\cos \theta-\alpha \sin \theta), \\
& B(\theta)=-c\left(2 \alpha \cos ^{3} \theta+3 \alpha \cos \theta \sin ^{2} \theta+\sin ^{3} \theta\right) \equiv c \bar{B}(\theta),
\end{aligned}
$$

for some smooth function $\bar{B}$ depending only on $\theta$ and $\alpha$, where

$$
\alpha=\cot \theta_{1}=-\frac{g}{c} \text {. }
$$

Notice that $\alpha$ takes values from 0 to $-\infty$ when $g$ goes from 0 to $+\infty$. Hence the angle $\theta_{1}$ goes from $\pi / 2$ to $\pi$. By substituting $\theta=0$ respectively $\theta=\theta_{1}$ in the above expressions one finds

$$
A(0)=A^{\prime}(0)=0, A^{\prime \prime}(0)=2 c>0 \quad \text { and } \quad B(0)=2 g=e=-2 \alpha c \geq 0,
$$

and respectively

$$
\begin{aligned}
& A\left(\theta_{1}\right)=0, A^{\prime}\left(\theta_{1}\right)=-c \sin \theta_{1}=\frac{-c}{\sqrt{1+\alpha^{2}}}<0, \\
& B\left(\theta_{1}\right)=\frac{c^{2}+2 g^{2}}{\sqrt{c^{2}+g^{2}}}=\frac{-c\left(2 \alpha^{4}+3 \alpha^{2}+1\right)}{\sqrt{\left(1+\alpha^{2}\right)^{3}}}<0,
\end{aligned}
$$

where we used the fact that $\sin \theta_{1}=\left(1+\alpha^{2}\right)^{-1 / 2}$ and $\theta_{1}$ lies in $[\pi / 2, \pi)$.

Clearly $A$ has 1 double zero at $\theta=0$ and 1 simple zero $\theta_{1}$, see Figure 4 Case 2 . Moreover we can describe the bifurcation of the phase portraits in terms of $\alpha$ and $c$. The parameter $\alpha$ determines the ray $\mathcal{R}_{1} \equiv\left\{\theta=\theta_{1}, r \geq 0\right\}$ on which singularities can occur besides on the ray $\mathcal{R}_{0} \equiv\{\theta=\pi, r \geq 0\}$. For fixed value of $\alpha$ the parameter $c$ determines the presence of 1,2 or 3 singularities on the rays $\mathcal{R}_{0}$ and $\mathcal{R}_{1}$. Indeed for fixed $\alpha$ the amplitude of the function $B$ grows linearly with $c$, see (40).

For a fixed but arbitrarily value $\alpha \leq 0$, we can describe the bifurcation of the phase portraits with respect to $c$. The values $\bar{B}(0) \equiv B_{0}$ and $\bar{B}\left(\theta_{1}\right) \equiv B_{1}$ are constants. Furthermore $B(0)$ and $B\left(\theta_{1}\right)$ linearly depend on $c$ and we can write

$$
B_{0, c} \equiv c B_{0} \quad \text { and } \quad B_{1, c} \equiv c B_{1} .
$$

Additionally it is easy to verify that $B_{1}<-B_{0}<0$, hence for all $c>0, B_{1, c}<-B_{0, c}<0$.

The bifurcation values $c_{1}=c_{1}(\alpha)$ and $c_{2}=c_{2}(\alpha)$ that determine the birth of singularities on the rays $\mathcal{R}_{1}$ and $\mathcal{R}_{0}$ are respectively given by

$$
c_{1}=-\frac{2}{B_{1}}
$$

and

$$
c_{2}=\frac{2}{B_{0}} .
$$

Case 2(a) If $0 \leq c<c_{1}$, then $B_{1, c}>-2$. The singularity at the origin is a global center of (2).

Case 2(b) If $c=c_{1}$, then $B_{1, c}=-2<-B_{0, c}$. There are 2 singularities, $(0,0)$ and $s^{1}=\left(\cos \theta_{1}, \sin \theta_{1}\right)$, both lying on $\mathcal{R}_{1}$.

Case 2(c) If $c_{1}<c<c_{2}$, then $B_{1, c}<-2<-B_{1, c}$. There are 3 singularities, $(0,0)$ and $s_{ \pm}^{1}$, on the ray $\mathcal{R}_{1}$ and there are no singularities outside this ray: $s_{-}^{1}$ is a saddle and $s_{-}^{1}$ is a center. The bifurcation of the phase portraits for $c$ passing through $c_{1}$ occurs analogously as in section 5.1, but this time the singularities are on ray $\mathcal{R}_{1}$ instead of the horizontal axis.

Case 2(d) If $c=c_{2}$, then $B_{1, c}<-2=-B_{0, c}$. There are 4 singularities, $(0,0)$ and $s_{ \pm}^{1}$ on the ray $\mathcal{R}_{1}$, and $s^{0}=(-1,0)$ on the ray $\mathcal{R}_{0}$. The type of the singularities $s_{-}^{1}$ (saddle) and $s_{-}^{1}$ (center) does not change, 
however their exact position on the ray $\mathcal{R}_{1}$ does change. The singularity $s^{0}$ is a degenerate cusp of order 1 (see Remark 4.1).

By Lemma 7 and Figure 11, we know that the Hamiltonian value at the saddle point $s_{-}^{1}$ is smaller than at the degenerate cusp singularity $s^{0}$, where $h^{0}=1 / 12$. Now because the Hamiltonian along $\mathcal{R}_{0}$ is strictly increasing with respect to $r$, there is a homoclinic loop through the degenerate cusp singularity that encloses the non-isolated periodic orbits surrounding the double homoclinic loop through $s_{-}^{1}$.

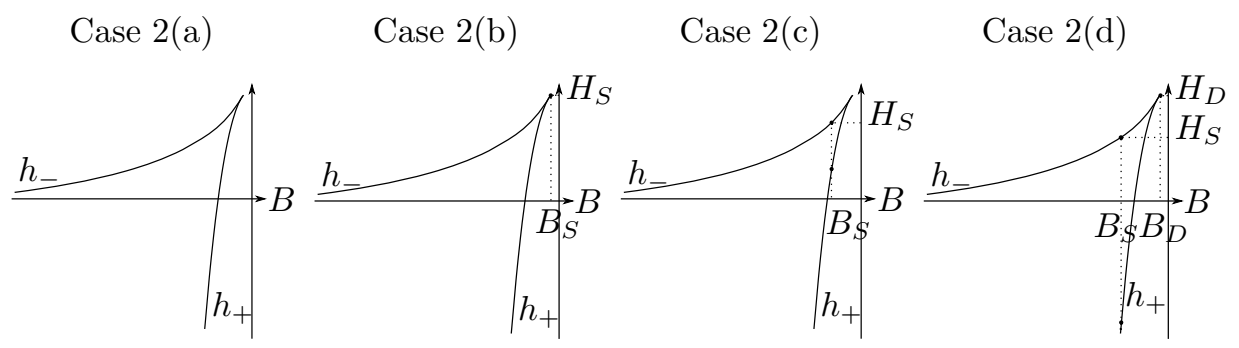

Case 2(e)

Case 2(f)

Case 2(g)
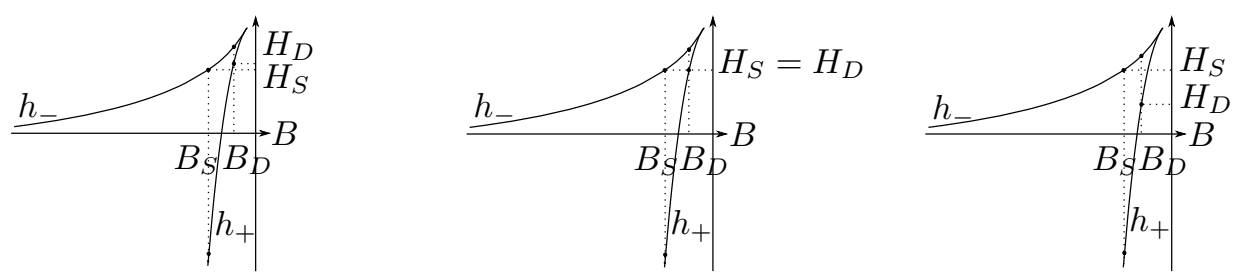

Fig. 11. Graphs of $h_{+}$and $h_{-}$for fixed value of the parameter $(\alpha, c)$; the parameter $c$ increases going from Case $2(\mathrm{a})$ to Case $2(\mathrm{~g})$.

If $c>c_{2}$, then there are 5 singularities, $(0,0)$ and $s_{ \pm}^{1}$ on the ray $\mathcal{R}_{1}$, and $(0,0)$ and $s_{ \pm}^{0}$ on the ray $\mathcal{R}_{0} ;$ the nilpotent cusps $s_{ \pm}^{0}$ are born from the degenerate cusp $s^{0}$.

Clearly the Hamiltonian value $h_{-}^{0}$ at the smallest cusp singularity is always bigger than the one $h_{-}^{1}$ at the saddle point along the ray $\mathcal{R}_{1}$. Therefore only a connection between the saddle singularity $s_{-}^{1}$ and the biggest cusp singularity $s_{+}^{0}$ is possible.

If $c$ now grows to $+\infty$, the corresponding values $B_{0, c}$ and $B_{1, c}$ decrease to $-\infty$ respecting their respective order, however as is illustrated in Figure 11, the relative order of the values of the corresponding Hamiltonian values changes at some value $c_{3}$, giving rise to the crossing of homoclinic connections and the appearance of a heteroclinic connection. Lemma 11 ensures the existence of this third bifurcation value $c_{3}=c_{3}(\alpha)>c_{2}$ for which

$$
h_{-}^{1}\left(c_{3}\right) \equiv h_{-}\left(c_{3} B_{1}\right)=h_{+}\left(-c_{3} B_{0}\right) \equiv h_{+}^{0}\left(c_{3}\right),
$$

and

$$
h_{-}^{1}(c)<h_{+}^{0}(c) \text { for } c_{2}<c<c_{3} \text { and } h_{-}^{1}(c)>h_{+}^{0}(c) \text { for } c>c_{3} .
$$

In particular only for $c=c_{3}$ there exists a connection between $s_{-}^{1}$ and $s_{+}^{0}$. Using the argument of continuity, the increasing of the Hamiltonian with respect to $r \geq 0$ and the corresponding graphs in Figure 11, we obtain the phase portraits in the cases $c>c_{2}$ as drawn in Figure 12.

Case 2(e) If $c_{2}<c<c_{3}$, i.e., $c>c_{2}$ and $h_{-}^{1}(c)<h_{+}^{0}(c)$, then $B_{1, c}<-B_{0, c}<-2$ and $h_{-}^{1}<h_{+}^{0}$. There are 5 singularities.

Case 2(f) If $c=c_{3}$, i.e., $c>c_{2}$ and $h_{-}^{1}(c)=h_{+}^{0}(c)$, then $B_{1, c}<-B_{0, c}<-2$ and $h_{-}^{1}=h_{+}^{0}$. There are 5 singularities.

Case 2(g) If $c>c_{3}$, i.e., $c>c_{2}$ and $h_{-}^{1}(c)>h_{+}^{0}(c)$, then $B_{1, c}<-B_{0, c}<-2$ and $h_{-}^{1}>h_{+}^{0}$. There are 5 singularities. 


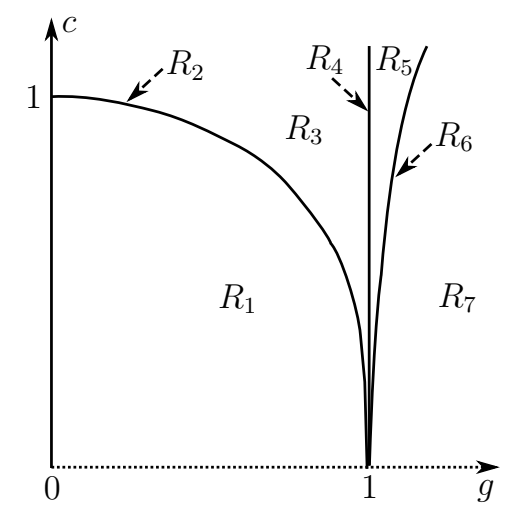

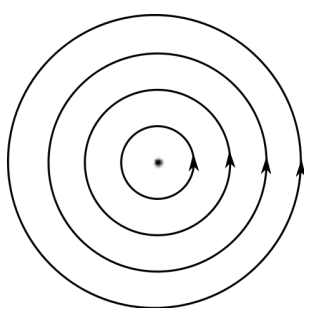

(a) $R_{1}$

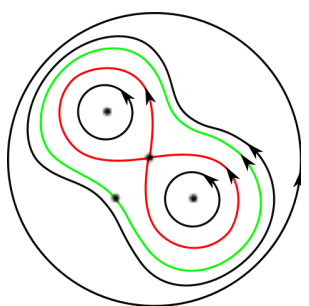

(d) $R_{4}, h_{+}^{1}<h_{-}^{1}<h^{0}=\frac{1}{12}$

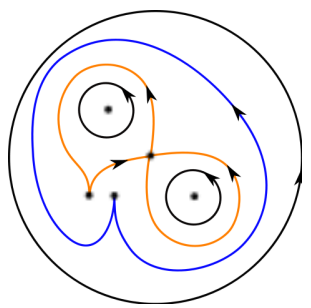

(f) $R_{6}, h_{+}^{1}<h_{-}^{1}=h_{+}^{0}<h_{-}^{1}<\frac{1}{12}$

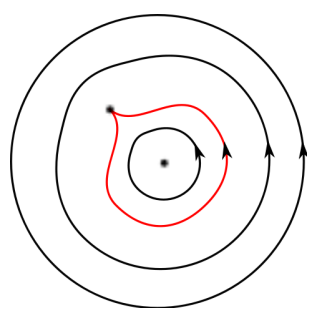

(b) $R_{2}, h^{1}=\frac{1}{12}$

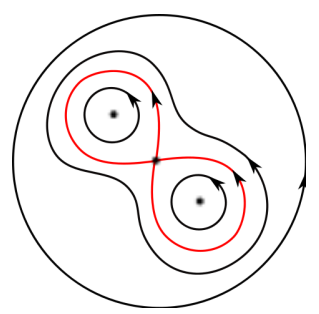

(c) $R_{3}, h_{+}^{1}<h_{-}^{1}<\frac{1}{12}$

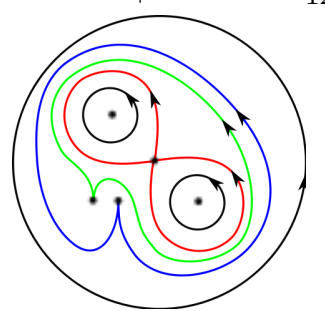

(e) $R_{5}, h_{+}^{1}<h_{-}^{1}<h_{+}^{0}<h_{-}^{0}<\frac{1}{12}$

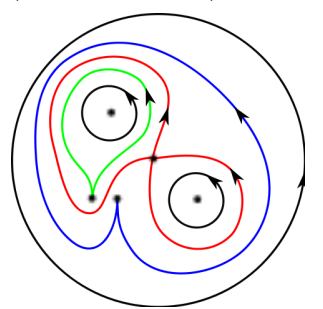

(g) $R_{7}, h_{+}^{1}<h_{+}^{0}<h_{-}^{1}<h_{-}^{0}<\frac{1}{12}$

Fig. 12. Bifurcation diagram of the global phase portraits of $(2)$ in the $(g, c)$-plane in Case $2, e-2 g=0, c \neq 0$ (see Theorem 2).

Using the relation between $\alpha$ and $(g, c)$ we can draw the bifurcation diagram of phase portraits in Case 2 in the $(g, c, e)$-space; by Lemma 2 it then suffices to present the bifurcation diagram to $g, c \geq 0$ in the plane $e=2 g$ as done in Figure 12. In this figure we distinguish between 7 regions which are denoted by $R_{1}, R_{2}, \ldots, R_{7}$ respectively for which the typical phase portraits in each region $R_{i}, i=1, \ldots, 7$, are respectively drawn.

In particular the explicit expression of the bifurcation curves can be computed. Solving (13) for $\theta=0$ and $\theta=\theta_{1}$ we obtain the value for the radius of the singularities $s_{ \pm}^{0}$ and $s_{ \pm}^{1}$ along the rays $\mathcal{R}_{0}$ for $c \geq c_{2}$ and $\mathcal{R}_{1}$ for $c \geq c_{1}$ respectively:

$$
r_{ \pm}^{0}=g \pm \sqrt{g^{2}-1} \quad \text { and } \quad r_{ \pm}^{1}=\frac{c^{2}+2 g^{2} \pm \sqrt{4 g^{4}+4 g^{2} c^{2}+c^{4}-4 g^{2}-4 c^{2}}}{2 \sqrt{g^{2}+c^{2}}}
$$

and for the corresponding Hamiltonian values at these singularities:

$$
\begin{aligned}
h_{ \pm}^{0}= & -\frac{1}{12}\left(g \pm \sqrt{g^{2}-1}\right)^{2}\left(-3+2 g^{2} \pm 2 g \sqrt{g^{2}-1}\right) \\
h_{ \pm}^{1}= & \frac{1}{96\left(c^{2}+g^{2}\right)^{2}}\left(-c^{2}-2 g^{2} \pm \sqrt{c^{4}+4 c^{2} g^{2}+4 g^{4}-4 c^{2}-4 g^{2}}\right)^{2} \\
& \left(-\left(c^{2}+2 g^{2}\right)^{2}+6\left(c^{2}+g^{2}\right) \pm\left(c^{2}+2 g^{2}\right) \sqrt{c^{4}+4 c^{2} g^{2}+4 g^{4}-4 c^{2}-4 g^{2}}\right) .
\end{aligned}
$$

Therefore the bifurcation curves defined in (42), (43) and (44) can be presented respectively as the following 
algebraic sets:

$$
\begin{aligned}
R_{2}= & \left\{(g, c): B_{1}=-2\right\}=\left\{(g, c): \widehat{R}_{2} \equiv 4 g^{4}+4 g^{2} c^{2}+c^{4}-4 g^{2}-4 c^{2}=0\right\}, \\
R_{4}= & \left\{(g, c): B_{0}=2\right\}=\left\{(g, c): \widehat{R}_{4} \equiv g-1=0\right\}, \\
R_{6}= & \left\{(g, c): h_{-}^{1}=H_{+}^{0}\right\}=\left\{(g, c): \widehat{R}_{6} \equiv 256 g^{10}+512 g^{8} c^{2}+352 g^{6} c^{4}\right. \\
& +96 g^{4} c^{6}+8 g^{2} c^{8}-768 g^{8}-1536 g^{6} c^{2}-960 g^{4} c^{4}-192 g^{2} c^{6}-9 c^{8} \\
& \left.+768 g^{6}+1536 g^{4} c^{2}+864 g^{2} c^{4}+96 c^{6}-256 g^{4}-512 g^{2} c^{2}-256 c^{4}=0\right\} .
\end{aligned}
$$

Taking into account the number of singularities, the phase portrait also is uniquely determined with respect to diffeomorphic equivalence in case the total number of singularities is less than five. When the maximum number of singularities is reached, five in this case, it is necessary to consider the value of the Hamiltonian at the singularities different from the origin. To be precise it suffices to consider only $h_{-}^{1}$ and $h_{+}^{0}$ to describe the bifurcation. For completeness in Figure 12 we indicate the relative value of the Hamiltonian values for all singularities.

This ends the proof of Theorems 2 and 5 in Case 2. Notice that $c \neq 0$ in Case 2, hence the Hamiltonian systems in Case 2 are not reversible and therefore there is nothing to prove for Theorem 4 in Case 2.

\subsection{Case 3: One simple ray - two complex rays}

By Lemma 2 and (19) we can assume without loss of generality that $e-2 g>0$. In this case the graphs of $A$ and $B$ look like in Figure 4 Case 3 . We have

$$
A(0)=0, \quad A^{\prime}(0)=e-2 g>0, \quad B(0)=e .
$$

By the assumption $g \geq 0$, it follows that $e>0$. Therefore all singularities lay on $\mathcal{R}_{0}$, which corresponds to the negative horizontal axis $\{(x, 0): x \leq 0\}$.

Using Proposition 1 and Lemma 9, for a fixed value of $g \geq 0$, a bifurcation occurs at $e=2$ diffeomorphically equivalent to the one that happens in case $e-2 g=c=0$.

For $e<2$ the phase portrait is a global center. For $e=2$ there is a cuspidal loop with cusp $s^{0}$ at $(-1,0)$; by Lemma 9 , it follows that $h^{0}=1 / 12$. For $e>2$ there appears the figure eight corresponding to a double homoclinic saddle loop at $s_{-}^{0}$; by Lemma 9 , it follows that $h_{+}^{0}<h_{-}^{0}<1 / 12$.

The bifurcations of the phase portraits in Case 3 with respect to $e$ are drawn in Figure 13. In particular these three phase portraits correspond with 1,2 or 3 critical points, respectively. This ends the proof of Case 3 of Theorems 2, 4 and 5 .

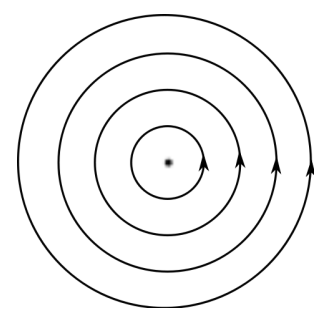

(a)

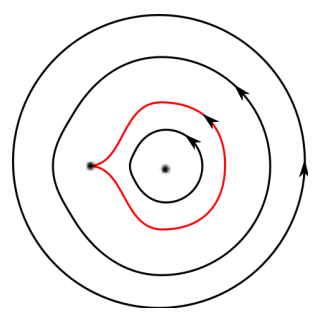

(b) $h^{0}=\frac{1}{12}$

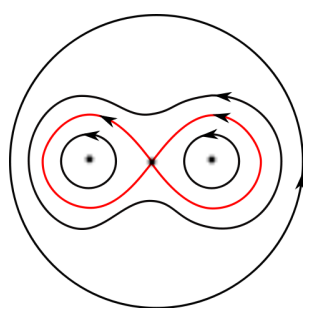

(c) $h_{+}^{0}<h_{-}^{0}<\frac{1}{12}$

Fig. 13. Phase portraits of (2) in Case $3, e-2 g \neq 0$ and $c^{2}-4 g(e-2 g)<0$ with (a) $0 \leq e<2$, (b) $e=2$ and (c) $e>2$ (see Theorem 2).

\subsection{Case 4: Three simples}

By Lemma 2 and (20) we can assume that $A^{\prime}(0)<0, g, c \geq 0$. As a consequence

$$
\lambda=-(e-2 g)=-A^{\prime}(0)>0 .
$$


Now the function $A$ can be rewritten as $A(\theta)=-\lambda \sin \theta(\cos \theta-\alpha \sin \theta)(\cos \theta-\beta \sin \theta)$, where

$$
\alpha=\frac{c+\sqrt{c^{2}+4 g \lambda}}{2 \lambda}>0 \quad \text { and } \quad \beta=\frac{c-\sqrt{c^{2}+4 g \lambda}}{2 \lambda} .
$$

Notice that under our assumptions necessarily $0 \leq-\beta \leq \alpha$. In particular $\alpha \beta=-\frac{g}{\lambda}, \alpha+\beta=\frac{c}{\lambda} \geq 0$. Hence $0<-\beta \leq \alpha$ for $g>0$, and $\beta=0$ for $g=0$. To a given value of the parameter $(\alpha, \beta, \lambda)$ there corresponds a unique $(g, c, e)$, given by

$$
e=-\lambda(1+2 \alpha \beta), \quad g=-\lambda \alpha \beta, \quad c=\lambda(\alpha+\beta) .
$$

To each parameter value $(g, c, e)$ of Case 4 there corresponds a unique parameter value $(\alpha, \beta, \lambda)$ with $0 \leq-\beta \leq \alpha$ defined by (46) and (47), and so in Case 4 we can describe the global phase portraits in terms of the new parameter $(\alpha, \beta, \lambda)$. Therefore we fix an arbitrary parameter $0 \leq-\beta \leq \alpha$ and we study the bifurcation of the global phase portraits in the 1-parameter subfamily $(2)$ with $(g, c, e)=$ $(-\lambda \alpha \beta, \lambda(\alpha+\beta),-\lambda(1+2 \alpha \beta))$ for $\lambda>0$.

In terms of the parameter $(\lambda, \alpha, \beta)$ the functions $A, B, H$ look like

$$
\begin{aligned}
A(\theta) & =-\lambda \sin \theta(\cos \theta-\alpha \sin \theta)(\cos \theta-\beta \sin \theta) \equiv \lambda \bar{A}(\theta), \\
B(\theta) & =-\lambda\left((1+2 \alpha \beta) \cos ^{3} \theta+3 \alpha \beta \cos \theta \sin ^{2} \theta+(\alpha+\beta) \sin ^{3} \theta\right) \equiv \lambda \bar{B}(\theta), \\
H(x, y) & =\frac{1}{2}\left(x^{2}+y^{2}\right)-\frac{\lambda}{3}(1+2 \alpha \beta) x^{3}-\lambda \alpha \beta x y^{2}-\frac{\lambda}{3}(\alpha+\beta) y^{3}+\frac{1}{4}\left(x^{2}+y^{2}\right)^{2},
\end{aligned}
$$

for some smooth functions $\bar{A}, \bar{B}$, that do not depend on $\lambda$. Define the angles

$$
0=\theta_{0}<\theta_{1} \equiv \theta_{1}(\alpha)<\theta_{2} \equiv \theta_{2}(\beta)<\pi,
$$

such that $\bar{A}\left(\theta_{i}\right), i=1,2$, satisfies $\cot \theta_{1}=\alpha$ and $\cot \theta_{2}=\beta$. Using the fact that $\cos \theta_{1}=\alpha / \sqrt{1+\alpha^{2}}$ and $\sin \theta_{1}=1 / \sqrt{1+\alpha^{2}}$, we can easily calculate that

$$
A^{\prime}(0)=-\lambda<0, \quad A^{\prime}\left(\theta_{1}\right)=\frac{(\alpha-\beta) \lambda}{\sqrt{1+\alpha^{2}}}>0 \quad \text { and } \quad A^{\prime}\left(\theta_{2}\right)=\frac{-(\alpha-\beta) \lambda}{\sqrt{1+\beta^{2}}}<0 .
$$

Clearly this case corresponds to the situation that $A$ has 3 simple zeroes in $[0, \pi)$.

By a straight-forward calculation we find the value of $B$ along the rays $\{\theta=0\},\left\{\theta=\theta_{1}\right\}$, and $\left\{\theta=\theta_{2}\right\}$ :

$$
\begin{gathered}
B(0)=\lambda \bar{B}(0)=-\lambda(1+2 \alpha \beta) \equiv \lambda \bar{B}_{0}(\alpha, \beta) \equiv B_{0}(\lambda), \\
B\left(\theta_{1}\right)=\lambda \bar{B}\left(\theta_{1}\right)=-\frac{\lambda\left(2 \alpha^{2} \beta+\alpha+\beta\right)}{\sqrt{1+\alpha^{2}}} \equiv \lambda \bar{B}_{1}(\alpha, \beta) \equiv B_{1}(\lambda), \\
B\left(\theta_{2}\right)=\lambda \bar{B}\left(\theta_{2}\right)=-\frac{\lambda\left(2 \alpha \beta^{2}+\alpha+\beta\right)}{\sqrt{1+\beta^{2}}} \equiv \lambda \bar{B}_{2}(\alpha, \beta) \equiv B_{2}(\lambda),
\end{gathered}
$$

for some smooth functions $\bar{B}_{i}, i=0,1,2$ depending only on $(\alpha, \beta)$.

For fixed $(\alpha, \beta)$ the graph of $A$ looks the same as the graph of $\bar{A}$ (resp. $B$ and $\bar{B}$ ); indeed it is only a rescaled copy with respect to the vertical coordinate. Denote by $\mathcal{R}_{i}$ the ray $\theta=\theta_{i}$ if $\bar{B}_{i} \equiv \bar{B}\left(\theta_{i}\right)<0$ and $\theta=\theta_{i}+\pi$ if $\bar{B}_{i} \equiv \bar{B}\left(\theta_{i}\right)>0, i=0,1,2$. Increasing $\lambda$ to $+\infty$ the values $\left|\bar{B}_{i}\right|$ pass subsequently through 2 and so singularities are born on the corresponding ray $\mathcal{R}_{i}$. The order according to which rays carry singularities for the first time is dictated by the relative order of $\left|\bar{B}_{i}\right|, i=0,1,2$. For instance suppose that $i_{j}, j \in\{0,1,2\}$ with

$$
\left|\bar{B}_{i_{0}}\right| \geq\left|\bar{B}_{i_{1}}\right| \geq\left|\bar{B}_{i_{2}}\right|,
$$

then singularities occur first on ray $\mathcal{R}_{i_{0}}$ (as soon as $\lambda=2 /\left|\bar{B}_{i_{0}}\right|$ ), next on ray $\mathcal{R}_{i_{1}}$ (as soon as $\lambda=2 /\left|\bar{B}_{i_{1}}\right|$ ) and finally on ray $\mathcal{R}_{i_{2}}$ (as soon as $\lambda=2 /\left|\bar{B}_{i_{2}}\right|$ ). Notice that we do not exclude the simultaneous appearance of singularities on different rays, thus when $\left|\bar{B}_{i_{0}}\right|=\left|\bar{B}_{i_{1}}\right|$, singularities appear simultaneously on rays $\mathcal{R}_{i_{0}}$ and $\mathcal{R}_{i_{1}}$. 
The type of the singularities on ray $\mathcal{R}_{i}$ is given by the sign of $\bar{A}^{\prime}\left(\theta_{i}\right), i=0,1,2$ as described by Proposition 1. If $\left|\bar{B}_{i}\right|<2$, neither of the rays $\theta=\theta_{i}$ and $\theta=\theta_{i}+\pi$ carry singularities but the center at the origin; if $\left|\bar{B}_{i}\right| \geq 2$, then $\operatorname{sgn}(\gamma)=\operatorname{sgn}\left(-\bar{A}^{\prime}\left(\theta_{i}\right) \bar{B}_{i}\right)=\operatorname{sgn}\left(\bar{B}_{i} \bar{B}_{i}^{\prime \prime}\right)$ characterizes the local phase portrait along the ray $\mathcal{R}_{i}$ for $i=0,1,2$.

Since $A$ has only simple zeroes the sign of

$$
A^{\prime}(0)=-\lambda<0,
$$

determines yet the sign of all $A^{\prime}\left(\theta_{i}\right)$, which is the opposite of the sign of $B_{i}^{\prime \prime}, i=1,2$. Indeed by a straightforward analysis one finds that

$$
(-1)^{i} B_{i}^{\prime \prime} \equiv(-1)^{i} B^{\prime \prime}\left(\theta_{i}\right)>0 .
$$

This formalizes the fact that $B$ has a local minimum at $\theta=\theta_{0}$, a local maximum at $\theta=\theta_{1}$, and a local minimum at $\theta=\theta_{2}$. Therefore essentially we need to distinguish between the following three cases:

$$
\begin{array}{r}
\text { Case } 4^{+}: \Pi>0, \text { Case } 4^{0}: \Pi=0 \text { and Case } 4^{-}: \Pi<0 \\
\text { where } \Pi \equiv \bar{B}_{0} \bar{B}_{1} \bar{B}_{2}
\end{array}
$$

Case $4^{0}$ corresponds to the case where at most two rays $\mathcal{R}_{i}, i=0,1,2$ can carry singularities. For Cases $4^{ \pm}$the typical graphs of $A$ and $B$ are drawn in Figure 14 .

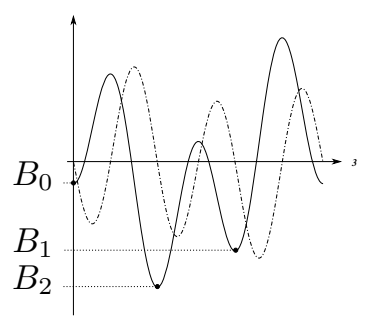

Case $4 \mathrm{Ai}$

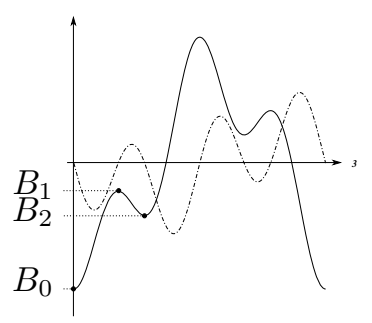

Case 4Bi

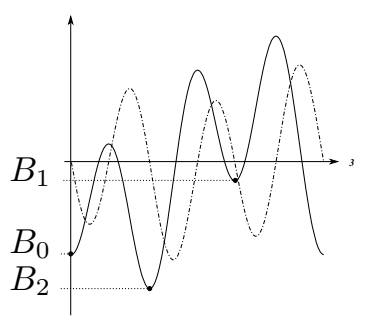

Case 4Aii

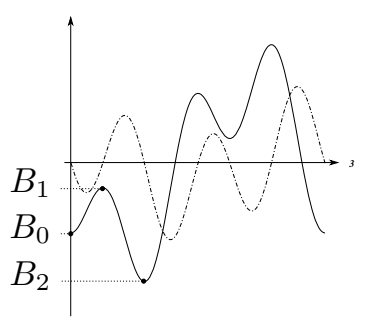

Case $4 \mathrm{Bii}$

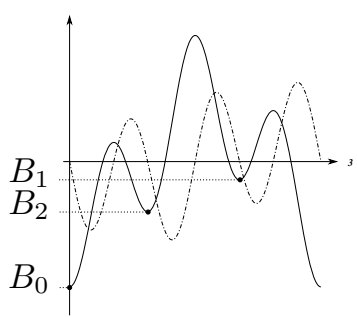

Case 4Aiii

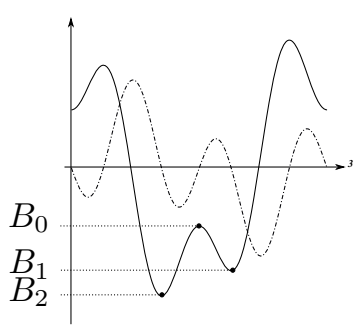

Case 4Biii

Fig. 14. Graphs of $A$ and $B$ in dashed and continuous line respectively for parameter values $(\alpha, \beta)$ in $\mathcal{A}$ or $\mathcal{B}$, divided in the cases like described on page 27. In these pictures the following representative values for the parameter value $(\alpha, \beta)$ are taken: $(1 / 2,-2 / 5)$ in Case $4 \mathrm{~A}(\mathrm{i}),(3 / 4,-2 / 5)$ in Case $4 \mathrm{~A}(\mathrm{ii}),(3 / 4,-3 / 5)$ in Case $4 \mathrm{~A}(\mathrm{iii}),(1 / 2,-1 / 5)$ in Case $4 \mathrm{~B}(\mathrm{i}),(1,-1 / 4)$ in Case $4 \mathrm{~B}(\mathrm{ii})$ and $(1,-3 / 4)$ in Case $4 \mathrm{~B}(\mathrm{iii})$.

In Case $4^{+}$necessarily $\bar{B}_{0}, \bar{B}_{2}<0$ and $\bar{B}_{1}>0$, i.e., $\mathcal{R}_{0}=\left\{\theta=\theta_{0}\right\}, \mathcal{R}_{2}=\left\{\theta=\theta_{2}\right\}$ and $\mathcal{R}_{1}=$ $\left\{\theta=\theta_{1}+\pi\right\}$, and $\bar{A}^{\prime}$ is negative along all rays $\mathcal{R}_{i}, i=0,1,2$. As a consequence the bifurcation diagram of the local phase portraits along these rays are diffeomorphically equivalent. In Case $4^{-}$necessarily $\bar{B}_{0} \bar{B}_{1}>0$ and $\bar{B}_{2}<0$, i.e.

$$
\begin{gathered}
\mathcal{R}_{0}=\left\{\theta=\theta_{0}\right\}, \mathcal{R}_{1}=\left\{\theta=\theta_{1}\right\} \text { and } \mathcal{R}_{2}=\left\{\theta=\theta_{2}\right\}, \text { or } \\
\mathcal{R}_{2}=\left\{\theta=\theta_{2}\right\}, \mathcal{R}_{0}=\left\{\theta=\theta_{0}+\pi\right\} \text { and } \mathcal{R}_{1}=\left\{\theta=\theta_{1}+\pi\right\} .
\end{gathered}
$$


If we define $\left(i_{0}, i_{1}, i_{2}\right)=(0,1,2)$ for $(52)$ and $\left(i_{0}, i_{1}, i_{2}\right)=(2,0,1)$ for $(53)$, then $\bar{A}^{\prime}$ is positive along the ray $\mathcal{R}_{i_{1}}$ and negative along the rays $\mathcal{R}_{i_{0}}$ and $\mathcal{R}_{i_{2}}$. In particular $\left|\bar{B}_{i_{1}}\right|=\min \left\{\left|\bar{B}_{i}\right|: i=0,1,2\right\}$. As a consequence first appear the singularities along the rays $\mathcal{R}_{i_{0}}$ and $\mathcal{R}_{i_{2}}$, and the bifurcation diagram of the local phase portraits along these rays are diffeomorphically equivalent. Next appear the singularities along ray $\mathcal{R}_{i_{1}}$, and the local phase portrait along this ray is oriented in the reverse direction with respect to the origin (e.g., the cusps along $\mathcal{R}_{i_{0}}$ and $\mathcal{R}_{i_{2}}$ point outwards while the cusp along $\mathcal{R}_{i_{1}}$ points inwards).

To describe the bifurcation of the global phase portrait of (2) with growing $\lambda$ to $+\infty$, we need to decide whether there is a connection between different singularities or not, and if not how the crossing of separatrices happens. This decision can be made based on the relative order of the Hamiltonian values at the saddle or cuspidal singularities (see Lemma 11). The result is summarized in Theorem 7. Statement (1) (resp. (2)) of Theorem 7 corresponds to the regular situation in Case $4^{+}$(resp. Case $4^{-}$), while statements (4), (5) and (8) (resp. (6)) correspond to the singular situations of Case $4^{+}$(resp. Case $4^{-}$) having some $\bar{B}_{i}=\bar{B}_{j}\left(\right.$ for $i \neq j$ ). Statements (3) and (7) correspond to Case $4^{0}$ having some $\bar{B}_{i}=0$.

To be precise we distinguish between the eight regions $\mathcal{A}, \mathcal{B}, \mathcal{C}, \mathcal{D}, \ldots, \mathcal{H}$, that represent parameters $(\alpha, \beta)$ with diffeomorphically different bifurcation diagrams, that in their turn can be split into subcases that are characterized by the order of the rays in which they start to carry singularities (see Figure 15). In this notation $(\alpha, \beta)$ belongs to $\mathcal{A}$ (resp. $\mathcal{B}$ ) if the condition (52) for Case $4^{+}$(resp. Case $4^{-}$) is satisfied and all $\left|\bar{B}_{i}\right|, i=0,1,2$ are mutually distinct; $\mathcal{C}, \mathcal{D}, \mathcal{E}$ and $\mathcal{H}$ (resp. $\mathcal{F}$ and $\mathcal{G}$ ) belong to the boundary of $\mathcal{A}$ (resp. $\mathcal{B})$. A precise algebraic definition of the sets $\mathcal{A}, \mathcal{B}, \ldots, \mathcal{H}$ is given below, but first we give an equivalent characterization of these regions in terms of the relative order of the $\left|\bar{B}_{i}\right|, i=0,1,2$ (see Figure 15); in particular we indicate the corresponding sign of $\Pi$ as defined in (52) and we indicate if the corresponding Hamiltonian system (2) is reversible by adding (rhs).

Case $4 \mathrm{~A}(\alpha, \beta) \in \mathcal{A}(\Pi>0)$

(i) $\bar{B}_{0}<\bar{B}_{2}<-\bar{B}_{1}<0$.

Case $4 \mathrm{~B}(\alpha, \beta) \in \mathcal{B}(\Pi<0)$

(i) $\bar{B}_{0}<\bar{B}_{2}<\bar{B}_{1}<0$.

Case $4 \mathrm{C}(\alpha, \beta) \in \mathcal{C}(\Pi=0)$

(i) $\bar{B}_{0}<\bar{B}_{2}<\bar{B}_{1}=0$.

Case $4 \mathrm{D}(\alpha, \beta) \in \mathcal{D}(\Pi>0)$

(i) $\bar{B}_{0}<\bar{B}_{2}=-\bar{B}_{1}<0$ (rhs).

Case $4 \mathrm{E}(\alpha, \beta) \in \mathcal{E}(\Pi>0)$

(i) $\bar{B}_{0}=\bar{B}_{2}<-\bar{B}_{1}<0$.

Case $4 \mathrm{~F}(\alpha, \beta) \in \mathcal{F}(\Pi<0)$

(i) $\bar{B}_{0}=\bar{B}_{2}<\bar{B}_{1}<0$.

Case $4 \mathrm{G}(\alpha, \beta) \in \mathcal{G}(\Pi=0)$

(i) $\bar{B}_{0}=\bar{B}_{2}<\bar{B}_{1}=0$.

Case $4 \mathrm{H}(\alpha, \beta) \in \mathcal{H}(\Pi>0)$

$\bar{B}_{0}=\bar{B}_{2}=-\bar{B}_{1}<0$ (rhs). (ii) $\bar{B}_{2}<\bar{B}_{0}<-\bar{B}_{1}<0$.

(ii) $\bar{B}_{2}<\bar{B}_{0}<\bar{B}_{1}<0$.

(ii) $\bar{B}_{2}<\bar{B}_{0}<\bar{B}_{1}=0$.

(ii) $\bar{B}_{2}<\bar{B}_{0}=-\bar{B}_{1}<0$.

(ii) $\bar{B}_{2}=-\bar{B}_{1}<\bar{B}_{0}<0$ (rhs).

(ii) $\bar{B}_{2}=-\bar{B}_{1}<-\bar{B}_{0}<0$ (rhs).

(ii) $\bar{B}_{2}=-\bar{B}_{1}<\bar{B}_{0}=0$ (rhs). (iii) $\bar{B}_{2}<-\bar{B}_{1}<\bar{B}_{0}<0$.

(iii) $\bar{B}_{2}<-\bar{B}_{1}<-\bar{B}_{0}<0$.

(iii) $\bar{B}_{2}<-\bar{B}_{1}<\bar{B}_{0}=0$.

The bifurcation curves in Figure 15 are obtained by straight-forward calculations. Clearly for parameter values $(\alpha, \beta)$ along the curve $1+2 \alpha \beta=0$ (resp. $2 \alpha^{2} \beta+\alpha+\beta=0$ ) with $0 \leq-\beta \leq \alpha, \alpha>0$, the value $\bar{B}_{0}$ (resp. $\bar{B}_{1}$ ) vanishes; as a consequence along this curve neither of the rays $\theta=\theta_{0}$ nor $\theta=\theta_{0}+\pi$ (resp; $\theta=\theta_{1}$ nor $\left.\theta=\theta_{1}+\pi\right)$ will carry singularities whatever the value of $\lambda$. Crossing this curve the ray $\mathcal{R}_{0}$ (resp. $\mathcal{R}_{1}$ ) that possible can carry singularities swaps over $\pi$ radians. A straight-forward calculation also implies that $\left|\bar{B}_{1}\right|=\left|\bar{B}_{2}\right|$ if and only if $|\alpha|=|\beta|$, and $\left|\bar{B}_{1}\right|=\left|\bar{B}_{0}\right|$ if and only if $\beta^{2}-2 \alpha \beta-1=0$, or since $0<-\beta<\alpha$

$$
\beta=\alpha-\sqrt{\alpha^{2}+1}
$$




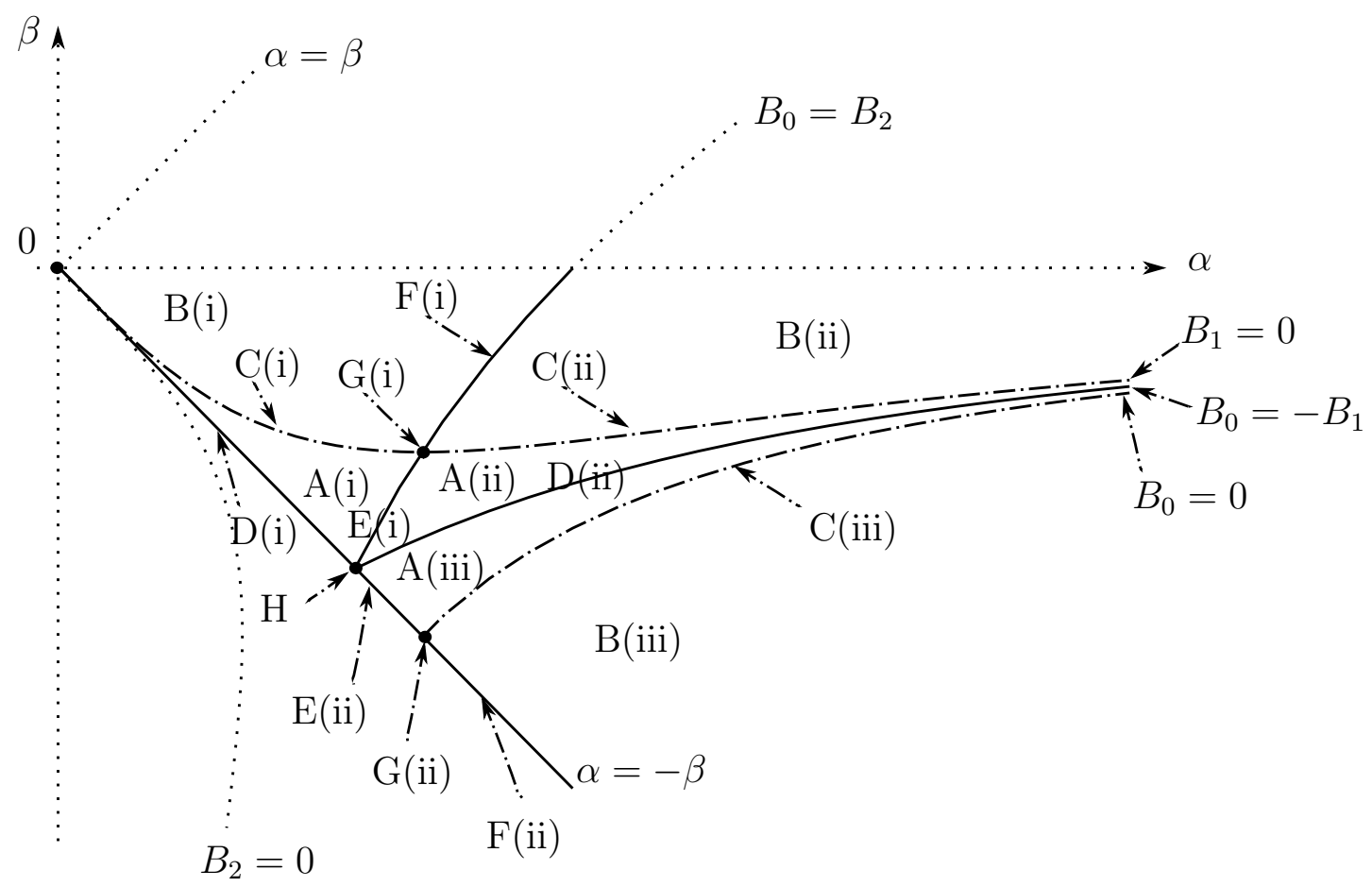

Fig. 15. The regions $\mathrm{A}(\mathrm{i}), 4 \mathrm{~A}(\mathrm{ii}), \ldots$ in the $(\alpha, \beta)$-plane correspond to Cases $4 \mathrm{~A}(\mathrm{i}), 4 \mathrm{~A}(\mathrm{ii}), \ldots$ as described on page 27 . When lifting the parameter $(\alpha, \beta)$ in the $(\alpha, \beta, \lambda)$-space and thus varying the value of $\lambda$, the corresponding phase portraits of (2) undergo the respective bifurcations presented in Figures 17, 18, 19, 20, 21, 22, 23, and 24.

by symmetry, it follows that $\left|\bar{B}_{2}\right|=\left|\bar{B}_{0}\right|$ if and only if $\alpha=\beta+\sqrt{\beta^{2}+1}$ since $\alpha>0$, or if

$$
\beta=\frac{\alpha^{2}-1}{2 \alpha} .
$$

As such the regions $\mathcal{A}, \mathcal{B}, \mathcal{C}, \mathcal{D}, \ldots, \mathcal{H}$ can algebraically be expressed as follows:

$$
\begin{aligned}
&(\alpha, \beta) \in \mathcal{A} \Longleftrightarrow \max \left\{0,2 \alpha^{2} \beta+\alpha\right\}<-\beta<\min \left\{\alpha, \frac{1}{2 \alpha}\right\} \text { and } \beta \neq \frac{\alpha^{2}-1}{2 \alpha} \text { and } \beta \neq \alpha-\sqrt{\alpha^{2}-1}, \\
&(\alpha, \beta) \in \mathcal{B} \Longleftrightarrow\left[0 \leq-\beta<\min \left\{\alpha, 2 \alpha^{2} \beta+\alpha\right\} \text { and } \beta \neq \frac{\alpha^{2}-1}{2 \alpha}\right] \text { or }\left[-\alpha<\beta<\alpha-\sqrt{\alpha^{2}+1}\right] \\
&(\alpha, \beta) \in \mathcal{C} \Longleftrightarrow\left[2 \alpha^{2} \beta+\alpha+\beta=0,0<\beta<-\alpha \text { and } \beta \neq \frac{\alpha^{2}-1}{2 \alpha}\right] \text { or }\left[-\frac{\sqrt{2}}{2}<\beta=-\frac{1}{2 \alpha}<0\right], \\
&(\alpha, \beta) \in \mathcal{D} \Longleftrightarrow\left[0<\alpha=-\beta<\frac{\sqrt{3}}{3}\right] \text { or }\left[-\frac{\sqrt{3}}{3}<\beta=\alpha-\sqrt{\alpha^{2}+1}<0\right], \\
&(\alpha, \beta) \in \mathcal{E} \Longleftrightarrow\left[-\frac{\sqrt{3}}{3}<\beta=\frac{\alpha^{2}-1}{2 \alpha}<-\frac{1}{2}\right] \text { or }\left[-\frac{\sqrt{2}}{2}<\beta=-\alpha<-\frac{\sqrt{3}}{3}\right], \\
&(\alpha, \beta) \in \mathcal{F} \Longleftrightarrow\left[-\frac{1}{2}<\beta=\frac{\alpha^{2}-1}{2 \alpha} \leq 0\right] \text { or }\left[\beta=-\alpha<\frac{\sqrt{2}}{2}\right], \\
& \mathcal{G}=\left\{\left(\frac{\sqrt{2}}{2},-\frac{\sqrt{2}}{4}\right),\left(\frac{\sqrt{2}}{2},-\frac{\sqrt{2}}{2}\right)\right\} \\
& \mathcal{H}=\left\{\left(\frac{\sqrt{3}}{3},-\frac{\sqrt{3}}{3}\right)\right\} .
\end{aligned}
$$


For fixed $(\alpha, \beta)$ the singularities $s_{ \pm}^{i} \equiv s_{ \pm}^{i}(\lambda)$ of $(2)$ on the ray $\mathcal{R}_{i}$ and the Hamiltonian $H$ at these singularities depend on $\lambda$ through $B\left(\theta_{i}\right)=\lambda \bar{B}_{i}, i=0,1,2$. We will denote

$$
h_{ \pm}^{i} \equiv h_{ \pm}^{i}(\lambda) \equiv h_{ \pm}\left(-\lambda\left|\bar{B}_{i}\right|\right) \text { and } h^{i} \equiv \frac{1}{12} .
$$

For simplicity of writing we will often omit this dependence if no confusion is possible.

Theorem 7. Consider the cubic Hamiltonian irs-system given by (2) and consider the parameter $(\alpha, \beta, \lambda)$ as defined in (46) and (47). Let $\bar{B}_{i}, i=0,1,2$ be defined by (48), (49) and (50) and let $i_{0}, i_{1}, i_{2} \in\{0,1,2\}$ be such that $\left|\bar{B}_{i_{0}}\right| \geq\left|\bar{B}_{i_{1}}\right| \geq\left|\bar{B}_{i_{2}}\right| \geq 0$. Let $\mathcal{A}, \mathcal{B}, \ldots, \mathcal{H}$ be the algebraic sets defined in $(56),(57), \ldots,(63)$. Then the bifurcation of the phase portraits of (2) with respect to $\lambda$ is summarized in

(1) Figure 17 for $(\alpha, \beta) \in \mathcal{A}$ and $\lambda_{0}^{\mathcal{A}}<\lambda_{1}^{\mathcal{A}}<\lambda_{2}^{\mathcal{A}}$ defined by $\lambda_{j}^{\mathcal{A}}=\lambda_{j}^{\mathcal{A}}(\alpha, \beta)=2 /\left|\bar{B}_{i_{j}}\right|, j=0,1,2$.

(2) Figure 18 for $(\alpha, \beta) \in \mathcal{B}$ and $\lambda_{0}^{\mathcal{B}}<\lambda_{1}^{\mathcal{B}}<\ldots<\lambda_{4}^{\mathcal{B}}$ with $\lambda_{j}^{\mathcal{B}}=\lambda_{j}^{\mathcal{B}}(\alpha, \beta)=2 /\left|\bar{B}_{i_{j}}\right|, j=0,1,2$, and $\lambda_{j}^{\mathcal{B}}=\lambda_{j}^{\mathcal{B}}(\alpha, \beta), j=3,4$ with $h_{-}\left(-\lambda_{3}^{\mathcal{B}}\left|\bar{B}_{i_{1}}\right|\right)=h_{+}\left(-\lambda_{3}^{\mathcal{B}}\left|\bar{B}_{i_{2}}\right|\right)$ and $h_{-}\left(-\lambda_{4}^{\mathcal{B}}\left|\bar{B}_{i_{0}}\right|\right)=h_{+}\left(-\lambda_{4}^{\mathcal{B}}\left|\bar{B}_{i_{2}}\right|\right)$.

(3) Figure 19 for $(\alpha, \beta) \in \mathcal{C}$ and $\lambda_{0}^{\mathcal{C}}<\lambda_{1}^{\mathcal{C}}$ with $\lambda_{j}^{\mathcal{C}}=\lambda_{j}^{\mathcal{C}}(\alpha, \beta)=2 /\left|\bar{B}_{i_{j}}\right|, j=0,1$.

(4) Figure 20 for $(\alpha, \beta) \in \mathcal{D}$ and $\lambda_{0}^{\mathcal{D}}<\lambda_{1}^{\mathcal{D}}$ with $\lambda_{j}^{\mathcal{D}}=\lambda_{j}^{\mathcal{D}}(\alpha, \beta)=2 /\left|\bar{B}_{i_{j}}\right|, j=0,1$.

(5) Figure 21 for $(\alpha, \beta) \in \mathcal{E}$ and $\lambda_{1}^{\mathcal{E}}<\lambda_{2}^{\mathcal{E}}$ by $\lambda_{j}^{\mathcal{E}}=\lambda_{j}^{\mathcal{E}}(\alpha, \beta)=2 /\left|\bar{B}_{i_{j}}\right|, j=1,2$.

(6) Figure 22 for $(\alpha, \beta) \in \mathcal{F}$ and $\lambda_{1}^{\mathcal{F}}<\lambda_{2}^{\mathcal{F}}<\lambda_{3}^{\mathcal{F}}$ with $\lambda_{j}^{\mathcal{F}}=\lambda_{j}^{\mathcal{F}}(\alpha, \beta)=2 /\left|\bar{B}_{i_{j}}\right|, j=1,2$, and $\lambda_{3}^{\mathcal{F}}=$ $\lambda_{3}^{\mathcal{F}}(\alpha, \beta)$ with $h_{-}\left(-\lambda_{3}^{\mathcal{F}}\left|\bar{B}_{i_{1}}\right|\right)=h_{+}\left(-\lambda_{3}^{\mathcal{F}}\left|\bar{B}_{i_{2}}\right|\right)$.

(7) Figure 23 for $(\alpha, \beta) \in \mathcal{G}$ and $\lambda_{1}^{\mathcal{G}}(\sqrt{2} / 2,-\sqrt{2} / 4)=1$ and $\lambda_{1}^{\mathcal{G}}(\sqrt{2} / 2,-\sqrt{2} / 4)=2 \sqrt{3}$. Moreover the bifurcation is described in terms of $g$.

(8) Figure 24 for $\alpha=-\beta=\sqrt{3} / 3$ and $\lambda_{0}^{\mathcal{H}}=6$. In this case $g=-e=\lambda / 3$ and $c=0$ and the cubic Hamiltonian irs-systems (2) are reversible. Moreover the bifurcation is described in terms of $g$.

Proof. [Proof of Theorem 7(2)] Locally near the rays the phase portrait is known from Proposition 1. Now we use the graphs of the Hamiltonian along the rays $\mathcal{R}_{i}, i=0,1,2$ to determine the relative position of the separatrices of the singularities that are not centers (Lemma 11). We only prove Theorem 7(2) in Case $4 \mathrm{~B}(\mathrm{ii})$, i.e., in case $(\alpha, \beta) \in \mathcal{B}$ and $\bar{B}_{2}<\bar{B}_{0}<\bar{B}_{1}<0$. Hence $\left(i_{0}, i_{1}, i_{2}\right)=(2,0,1)$ and $\mathcal{R}_{j}=\left\{\theta=\theta_{i_{j}}\right\}, 0 \leq$ $j \leq 2$. The other cases are analogous.

Figure 16 presents the bifurcation of the Hamiltonian at the singularities and the corresponding phase portrait with increasing $\lambda$. On the left of these figures, there are the graphs of the Hamiltonian values $h_{+}$and $h_{-}$at the singularities $r_{+}$and $r_{-}$in function of $B$ (and so in terms of $-\lambda$, as $\left|B_{i}(\lambda)\right|$ grows linearly with $\lambda$ ). The Hamiltonian values $h_{ \pm}^{i}, h^{i}, i=0,1,2$ that correspond to saddle or cusp singularities are indicated by a big bullet and the Hamiltonian values $h_{ \pm}^{i}, i=0,1,2$ that correspond to a center are indicated by a thin bullet. In the middle of these figures the graphs of the Hamiltonian $\bar{H}_{\lambda \bar{B}_{i}}$ along the rays $\mathcal{R}_{i}, i=0,1,2$ are presented as a function of $r$, in dashed, dotted, point-dashed line respectively. Again the value of the Hamiltonian is indicated at the saddle and cusp points by a big bullet and at the center points by a thin bullet. Furthermore for $i=0,1,2$ and for $\lambda$ big enough such that the singularities $s_{ \pm}^{i}$ or $s^{i}$ exist along $\mathcal{R}_{i}$ the horizontal lines $\Gamma_{i}$, defined by $\left\{H=h_{-}^{i}(\lambda)\right\}$, are drawn in dashed, dotted, point-dashed line respectively (value of the Hamiltonian at the saddle or cusp point along $\mathcal{R}_{i}$ ).

If such a horizontal line $\Gamma_{j}, j=0,1,2$ intersects the graph of $H_{\lambda \bar{B}_{i}}, i=0,1,2(i \neq j)$ for a value $r=r^{*}$, then the level curve $\Gamma_{j}$ also intersects the ray $\mathcal{R}_{i}$ in the phase portrait at distance $r^{*}$. Comparing the relative position of the intersection points and the critical points of the Hamiltonian $\bar{H}_{\lambda \bar{B}_{i}}$ along the ray $\mathcal{R}_{i}, i=0,1,2$, we can complete the phase portrait as shown in the right of Figure 16, where the orbits in dashed, pointed resp. point-dashed line correspond to level curves of the Hamiltonian at the saddle or cusp singularity if occurring along $\mathcal{R}_{i}, i=0,1$ resp. 2 . The level curve $\Gamma_{j}$ intersects $\mathcal{R}_{i}$ in a point $s^{*}$ with $\left|s^{*}\right|=r^{*}$ and $s^{*}$ lies on the ray $\mathcal{R}_{i}$ between $(0,0)$ and $s_{-}^{i}, s_{-}^{i}$ and $s_{+}^{i}$ or between $s_{+}^{i}$ and $+\infty\left(\right.$ or $(0,0)$ and $s^{i}$ or between $s^{i}$ and $\left.+\infty\right)$. In the same way by comparing the order of the intersections of the horizontal lines $\Gamma_{i}, i=0,1,2$ and the graph of $\bar{H}_{\lambda \bar{B}_{j}}$ along a fixed ray $\mathcal{R}_{j}$, we know the corresponding order of intersections of the level curves $\Gamma_{i}, i=0,1,2$ with $\mathcal{R}_{j}$, and so by Lemma 11 we can derive the configuration of the 
saddle and cusp connections. In this way and by use of a continuity argument we describe the bifurcation for increasing $\lambda$.

It can be seen from Figure 16 that in Case $4(b)$ there are 11 possible configurations for the singularities on the rays $\mathcal{R}_{i}, i=0,1,2$ and the Hamiltonian values at the saddle points, that depend on the relative values $\bar{B}_{2}<\bar{B}_{0}<\bar{B}_{1}<0$ with respect to -2 and the relative values of the Hamiltonian at the saddle points.

(a) If $\lambda<\lambda_{0}^{\mathcal{B}}$, then $B_{2}(\lambda)>-2$ and hence the origin is a global center, see Figure 16(a).

(b) If $\lambda=\lambda_{0}^{\mathcal{B}}$, then $B_{2}(\lambda)=-2$. Hence there is one singularity on the ray $\mathcal{R}_{2}$ of cuspidal type with Hamiltonian value $h^{2}\left(\lambda_{0}^{\mathcal{B}}\right)=1 / 12$. The Hamiltonian along the rays $\mathcal{R}_{i}, i=0,1,2$ is strictly increasing with the radial value $r$, therefore the phase portrait presents a center at the origin bounded by a cuspidal loop, that is pointing outwards (since $\bar{A}^{\prime}\left(\theta_{2}\right)<0$ ), and a center at infinity that reaches to the cuspidal loop, see Figure 16(b).

(c) If $\lambda_{0}^{\mathcal{B}}<\lambda<\lambda_{1}^{\mathcal{B}}$, then $B_{2}(\lambda)<-2<B_{0}(\lambda)$. Hence the ray $\mathcal{R}_{2}$ carries two singularities, a saddle and a center. Outside this ray there is only the center in the origin. By studying the Hamiltonian along the rays $\mathcal{R}_{i}, i=0,1,2$ in function of $r$, we conclude that the phase portrait exhibits a figure eight connection at the saddle point of which one loop encircles the center at the origin and the other loop encircles the center at $s_{+}^{2}$, see Figure 16(c). Notice that the value of the Hamiltonian at the saddle point is strictly smaller than $1 / 12$.

(d) If $\lambda=\lambda_{1}^{\mathcal{B}}$, then $B_{0}(\lambda)=-2$. By continuous dependence on $\lambda$ the local phase portrait near the ray $\mathcal{R}_{2}$ is unchanged, and along the ray $\mathcal{R}_{0}$ a cuspidal singularity is born. By studying the Hamiltonian that is increasing along the ray $\mathcal{R}_{0}$ we conclude that there is a cuspidal loop pointing outwards encircling the figure eight determined by the saddle point at $\mathcal{R}_{2}$, see Figure $16(\mathrm{~d})$.

(e) If $\lambda_{1}^{\mathcal{B}}<\lambda<\lambda_{2}^{\mathcal{B}}$, then $B_{0}(\lambda)<-2<B_{1}(\lambda)$. Locally along $\mathcal{R}_{2}$ the phase portrait is unchanged and locally along $\mathcal{R}_{0}$ the cusp singularity has split up in a center $s_{+}^{0}$ and a saddle singularity $s_{-}^{0}$. Analyzing the Hamiltonian, shown in the corresponding graphs of Figure 16(e), one finds that the phase portrait exists of a topological lemniscate through $s_{-}^{0}$, that bounds the center at $s_{+}^{0}$ by one of its loops and it bounds the non-isolated periodic orbits encircling the lemniscate through $s_{-}^{2}$ by the other loop. The lemniscate through $s_{-}^{0}$ itself is encircled by non-isolated periodic orbits reaching to the center at $\infty$. Notice that for all $\lambda>\lambda_{1}^{\mathcal{B}}$, it follows that $B_{2}(\lambda)<B_{0}(\lambda)<-2$ and hence $h_{-}^{2}(\lambda)<h_{-}^{1}(\lambda)$. Therefore for increasing $\lambda>\lambda_{1}^{\mathcal{B}}$ the relative position of the saddle connections at $s_{-}^{0}$ and $s_{-}^{2}$ will be preserved.

(f) If $\lambda=\lambda_{2}^{\mathcal{B}}$, then $B_{1}(\lambda)=-2$ and hence a cusp singularity is born along $\mathcal{R}_{1}$ pointing inwards. By an analysis based on the Hamiltonian, see Figure 16(f), it follows that the phase portrait has a cuspidal loop bounding the non-isolated periodic orbits encircling the lemniscate through $s_{-}^{0}$.

(g, h, i, j, k) If $\lambda>\lambda_{2}^{\mathcal{B}}$, then $B_{1}(\lambda)<-2$ and we clearly have that $h_{-}^{2}(\lambda)<h_{-}^{0}(\lambda)<h_{-}^{1}(\lambda)$. The cusp singularity $s^{1}$ has split up into a center and a saddle singularity like happened on rays $\mathcal{R}_{i}, i=0,2$. However this time the smallest singularity $s_{-}^{1}$ is the center point and the biggest one $s_{+}^{1}$ is the saddle point. In particular the Hamitonian value $h_{+}^{1}(\lambda)$ at the saddle $s_{+}^{1}$ will decrease with increasing $\lambda \in\left(\lambda_{2}^{\mathcal{B}},+\infty\right)$ from $1 / 12$ to $-\infty$, permitting the crossing of separatrices with increasing $\lambda$, see Figure 16. By Lemma 11, there exist $\lambda_{3}^{\mathcal{B}}$ and $\lambda_{4}^{\mathcal{B}}$ such that

$$
\begin{array}{cc}
h_{-}^{0}\left(\lambda_{3}^{\mathcal{B}}\right)=h_{+}^{1}\left(\lambda_{3}^{\mathcal{B}}\right), \quad h_{-}^{2}\left(\lambda_{4}^{\mathcal{B}}\right)=h_{+}^{1}\left(\lambda_{4}^{\mathcal{B}}\right), \quad h_{-}^{0}(\lambda)<h_{+}^{1}(\lambda) \text { for } \lambda_{2}^{\mathcal{B}}<\lambda<\lambda_{3}^{\mathcal{B}}, \\
h_{-}^{2}(\lambda)<h_{+}^{1}(\lambda)<h_{-}^{0}(\lambda) \text { for } \lambda_{3}^{\mathcal{B}}<\lambda<\lambda_{4}^{\mathcal{B}} \quad \text { and } \quad h_{+}^{1}(\lambda)<h_{-}^{2}(\lambda) \text { for } \lambda>\lambda_{4}^{\mathcal{B}} .
\end{array}
$$

(g) If $\lambda_{2}^{\mathcal{B}}<\lambda<\lambda_{3}^{\mathcal{B}}$, then from an analysis based on the Hamiltonian, see Figure 16, it follows that the level curve $\Gamma_{1}$ through $s_{+}^{1}$ takes topologically the form of Cayley's Sextic: with the center at $s_{-}^{1}$ bounded by its interior loop and the non-isolated periodic orbits encircling the topological lemniscate through $s_{-}^{0}$ bounded by its outermost loop.

(h) If $\lambda=\lambda_{3}^{\mathcal{B}}$, then from an analysis based on the Hamiltonian, see Figure 16, and by Lemma 11, it follows that a connection between the separatrices of the saddles $s_{-}^{0}$ and $s_{+}^{1}$ exist. The rest is unchanged 


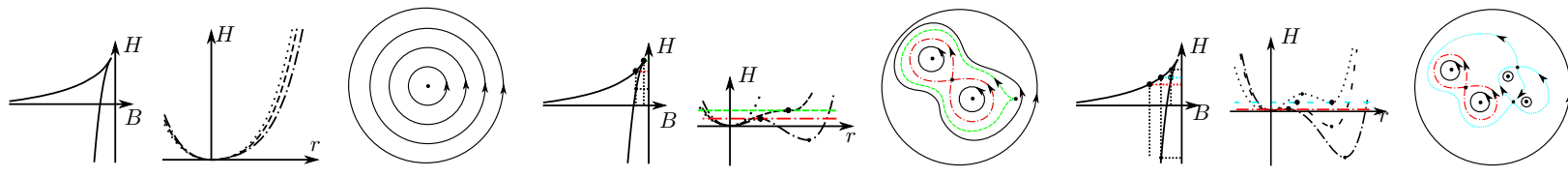

(d)

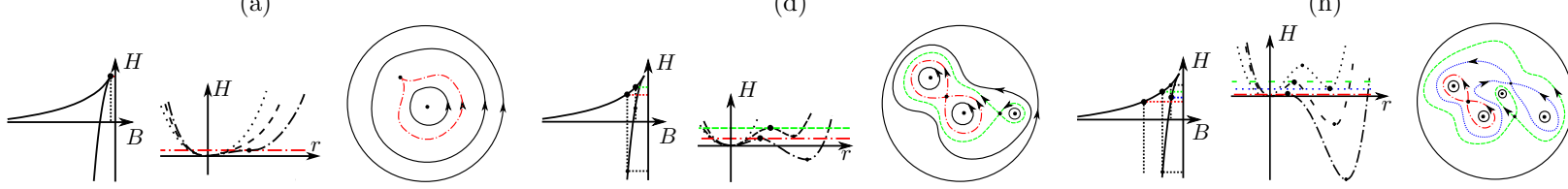

(b)

(e)
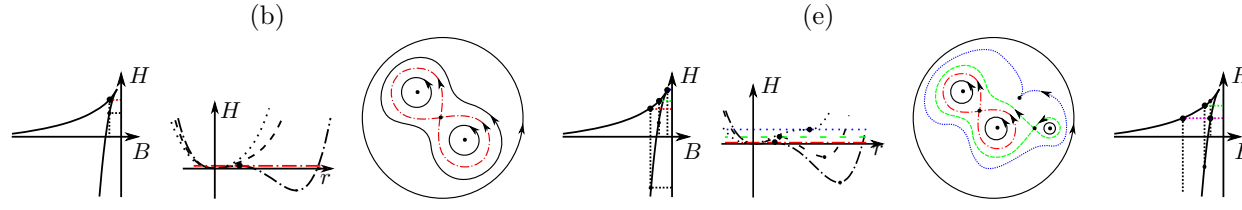

(i)

(c)

(f)
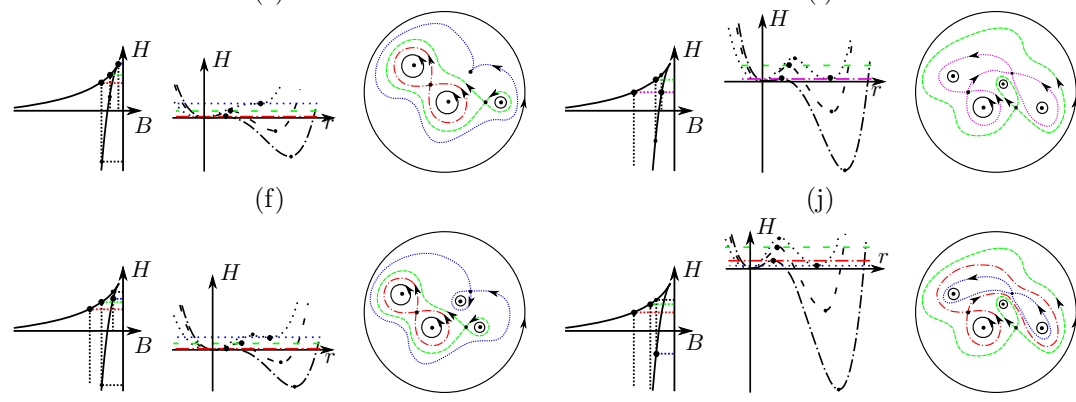

(g)

(k)

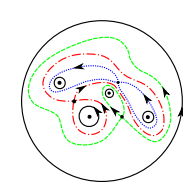

Fig. 16. Graphical bifurcation analysis of the phase portraits of (2) in Case 4B(ii), see Theorem 7(2). 
with respect to (g) by continuous dependence on $\lambda$.

(i) If $\lambda_{3}^{\mathcal{B}}<\lambda<\lambda_{4}^{\mathcal{B}}$, then from an analysis based on the Hamiltonian, see Figure 16, and by Lemma 11, it follows that the connection between the separatrices of the saddles $s_{-}^{0}$ and $s_{+}^{1}$ is broken and the order in which the separatrices intersect the ray $\mathcal{R}_{1}$ is reversed with respect to parameter values $\lambda_{2}^{\mathcal{B}}<\lambda<\lambda_{3}^{\mathcal{B}}$, see $(\mathrm{g})$. The rest is unchanged with respect to (h) by continuous dependence on $\lambda$.

(j) If $\lambda=\lambda_{4}^{\mathcal{B}}$, then from an analysis based on the Hamiltonian, see Figure 16, and by Lemma 11, it follows that a connection between the separatrices of the saddles $s_{-}^{2}$ and $s_{+}^{1}$ exist. The rest is unchanged with respect to (i) by continuous dependence on $\lambda$.

(k) If $\lambda>\lambda_{4}^{\mathcal{B}}$, then from an analysis based on the Hamiltonian, see Figure 16, and by Lemma 11, it follows that the connection between the separatrices of the saddles $s_{-}^{2}$ and $s_{+}^{1}$ is broken and the order in which the separatrices intersect the ray $\mathcal{R}_{1}$ is reversed with respect to parameter values $\lambda_{3}^{\mathcal{B}}<\lambda<\lambda_{4}^{\mathcal{B}}$, see (g). The rest is unchanged with respect to (j) by continuous dependence on $\lambda$.

\begin{tabular}{clll} 
Figure & $\lambda$ & Hamiltonian & \# \\
\hline (a) & $0 \leq \lambda<\lambda_{0}^{\mathcal{A}}$ & & 1 \\
(b) & $\lambda=\lambda_{0}^{\mathcal{A}}$ & $h^{i_{0}}=1 / 12$ & 2 \\
(c) & $\lambda_{0}^{\mathcal{A}}<\lambda<\lambda_{1}^{\mathcal{A}}$ & $h_{-}^{i_{0}}<1 / 12$ & 3 \\
(d) & $\lambda=\lambda_{1}^{\mathcal{A}}$ & $h_{-}^{i_{0}}<h^{i_{1}}=1 / 12$ & 4 \\
(e) & $\lambda_{1}^{\mathcal{A}}<\lambda<\lambda_{2}^{\mathcal{A}}$ & $h_{\overline{i_{0}}}<h_{\overline{-}}^{i_{1}}<1 / 12$ & 5 \\
(f) & $\lambda=\lambda_{2}^{\mathcal{A}}$ & $h_{-}^{i_{0}}<h_{-}^{i_{1}}<h^{i_{2}}=1 / 12$ & 6 \\
(g) & $\lambda>\lambda_{2}^{\mathcal{A}}$ & $h_{-}^{i_{0}}<h_{-}^{i_{1}}<h_{-}^{i_{2}}<1 / 12$ & 7
\end{tabular}

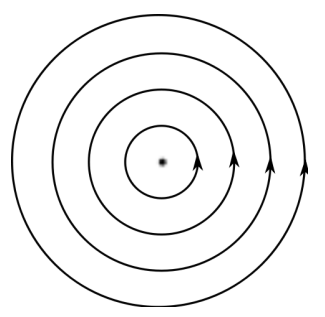

(a)

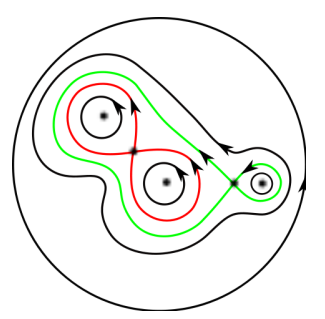

(e)

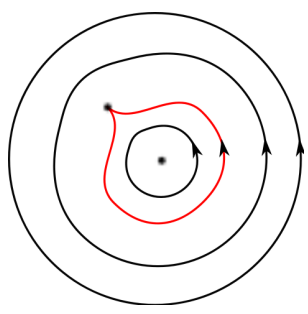

(b)

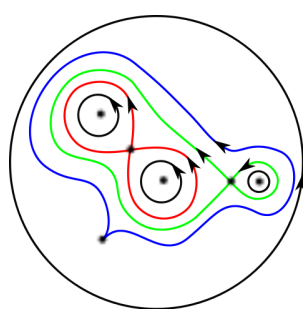

(f)

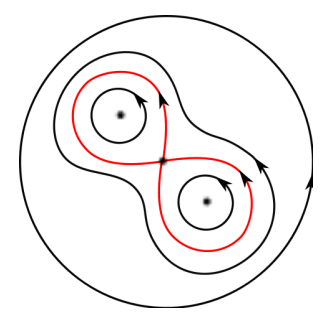

(c)

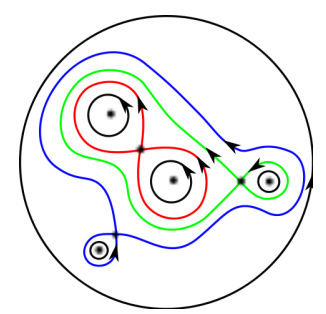

(g)

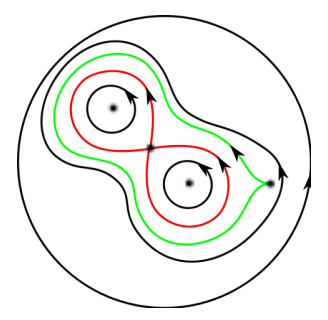

(d)

Fig. 17. Bifurcation of phase portraits of (2) in Case 4A (see Theorem 7(1)).

Proof. [Proof of Theorem 7(1)] Since in Case 4A the saddle points along $\mathcal{R}_{i_{j}}, j=0,1,2$ correspond to the smallest ones, $s_{-}^{i_{j}}, j=0,1,2$ the bifurcation of the phase portraits with respect to increasing $\lambda<\lambda_{2}^{A}$ in Case $4 \mathrm{~A}$ is analogous to the bifurcation of the phase portraits in Case $4 \mathrm{~B}$ for $\lambda<\lambda_{2}^{B}$. For $\lambda=\lambda_{2}^{\mathcal{A}}$ a cusp singularity $s^{i_{2}}$ is born along $\mathcal{R}_{i_{2}}$, this time pointing outwards. For $\lambda>\lambda_{2}^{\mathcal{A}}$ this cusp singularity splits into a saddle singularity $s_{-}^{i_{2}}$ and a center singularity $s_{+}^{i_{2}}$, giving rise to a homoclinic saddle connection at $s_{-}^{i_{2}}$ surrounding the center at $s_{+}^{i_{2}}$ and the non-isolated periodic orbits surrounding the lemniscate at $s_{-}^{i_{1}}$. Since 


\begin{tabular}{|c|c|c|}
\hline Figure & $\lambda$ & Hamiltonian \\
\hline (a) & $0 \leq \lambda<\lambda_{0}^{\mathcal{B}}$ & \\
\hline (b) & $\lambda=\lambda_{0}^{\mathcal{B}}$ & $h^{i_{0}}=1 / 12$ \\
\hline (c) & $\lambda_{0}^{\mathcal{B}}<\lambda<\lambda_{1}^{\mathcal{B}}$ & $h_{-}^{i_{0}}<1 / 12$ \\
\hline (d) & $\lambda=\lambda_{1}^{\mathcal{B}}$ & $h_{-}^{i_{0}}<h^{i_{1}}=1 / 12$ \\
\hline (e) & $\lambda_{1}^{\mathcal{B}}<\lambda<\lambda_{2}^{\mathcal{B}}$ & $h_{-}^{i_{0}}<h_{-}^{i_{1}}<1 / 12$ \\
\hline (f) & $\lambda=\lambda_{2}^{\mathcal{B}}$ & $h_{-}^{\overline{i_{0}}}<h_{-}^{\overline{i_{1}}}<h^{i_{2}}=1 / 12$ \\
\hline$(g)$ & $\lambda_{2}^{\mathcal{B}}<\lambda<\lambda_{3}^{\mathcal{B}}$ & $h_{-}^{i_{0}}<h_{-}^{i_{1}}<h_{+}^{i_{2}}<h_{-}^{i_{2}}<1 / 12$ \\
\hline (h) & $\lambda=\lambda_{3}^{\mathcal{B}}$ & $h_{-}^{i_{0}}<h_{-}^{i_{1}}=h_{+}^{i_{2}}<h_{-}^{i_{2}}<1 / 12$ \\
\hline (i) & $\lambda_{3}^{\mathcal{B}}<\lambda<\lambda_{4}^{\mathcal{B}}$ & $h_{-}^{i_{0}}<h_{+}^{i_{2}}<h_{-}^{i_{1}}<h_{-}^{i_{2}}<1 / 12$ \\
\hline (j) & $\lambda=\lambda_{4}^{\mathcal{B}}$ & $h_{-}^{i_{0}}=h_{+}^{i_{2}}<h_{-}^{i_{1}}<h_{-}^{i_{2}}<1 / 12$ \\
\hline$(\mathrm{k})$ & $\lambda>\lambda_{4}^{\mathcal{B}}$ & $h_{+}^{i_{2}}<h_{-}^{i_{0}}<h_{-}^{i_{1}}<h_{-}^{i_{2}}<1 / 12$ \\
\hline
\end{tabular}

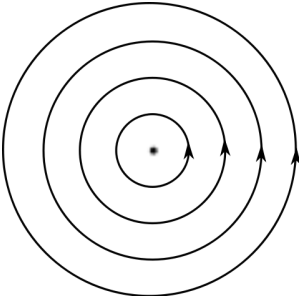

(a)

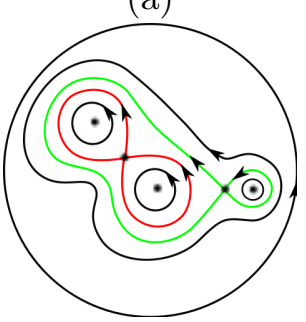

(e)

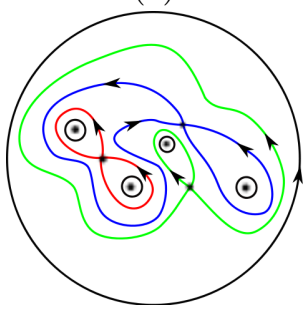

(i)

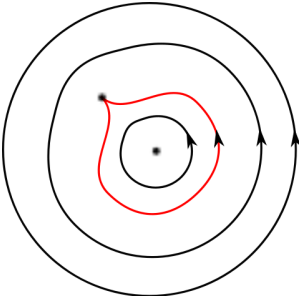

(b)

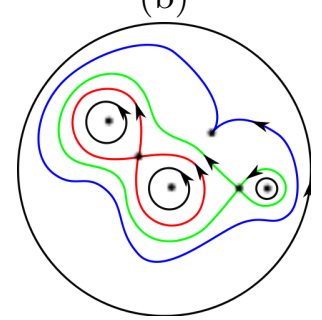

(f)

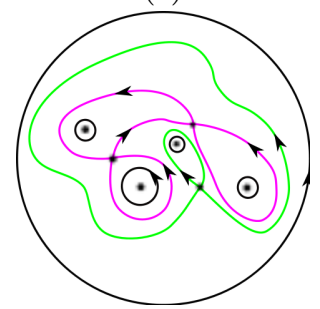

(j)

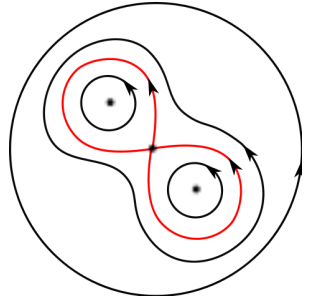

(c)

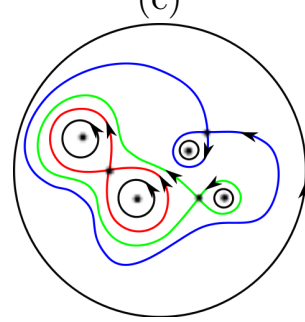

(g)

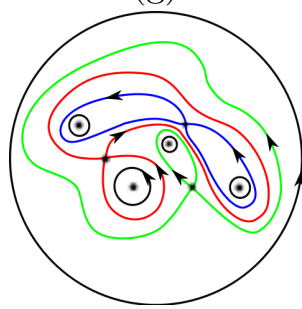

$(\mathrm{k})$

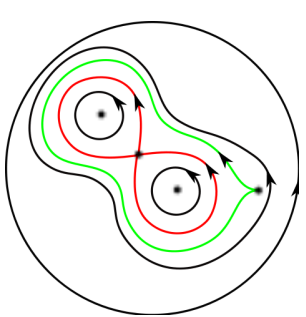

(d)

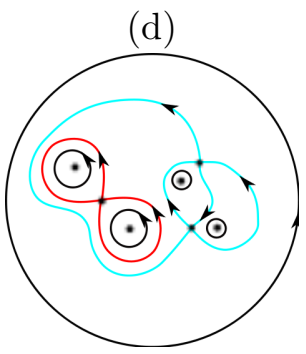

(h)

Fig. 18. Bifurcation of phase portraits of (2) in Case 4B (see Theorem 7(2)).

for $\lambda>\lambda_{2}^{\mathcal{A}}$ the order of the Hamiltonian values at the saddle points is stable, $h_{-}^{i_{0}}<h_{-}^{i_{1}}<h_{-}^{i_{2}}$, no crossing of the separatrices at the different saddle points $s_{-}^{i}, i=0,1,2$ is possible. Hence no further bifurcation with increasing $\lambda$ will appear.

Proof. [Proof of Theorem 7(3)] In Case 4C since $\bar{B}_{i_{2}}=0$ one also has that $B_{i_{2}}(\lambda)=0$ for all $\lambda$. Hence no singularities appear on ray $\mathcal{R}_{i_{2}}$, for any value of the parameter $\lambda$. Therefore and since the saddle points of $\mathcal{R}_{i_{0}}$ and $\mathcal{R}_{i_{1}}$ correspond to the smallest ones, $s_{-}^{i_{0}}$ and $s_{-}^{i_{1}}$, the bifurcation of the phase portraits with respect to increasing $\lambda$ in Case $4 \mathrm{C}$ is analogous to the bifurcation of the phase portraits in Case $4 \mathrm{~B}$ for $\lambda<\lambda_{2}^{B}$. 


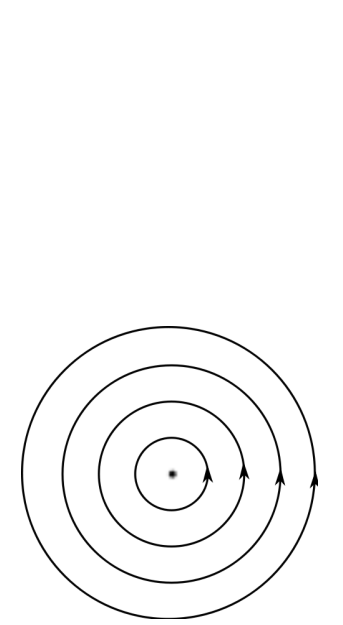

(a)

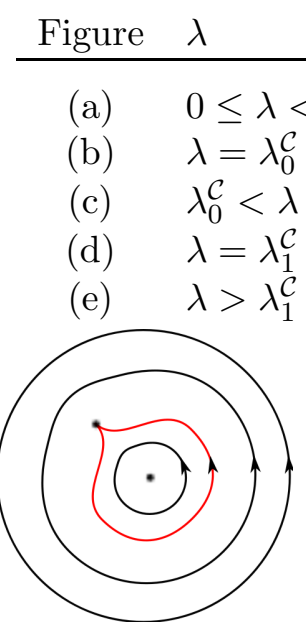

(b)

Hamiltonian

$\# s$

1

2

3

$h^{i_{0}}=1 / 12$

$h_{-}^{i_{0}}<1 / 12$

$h_{-}^{i_{0}}<h^{i_{1}}=1 / 12 \quad 4$

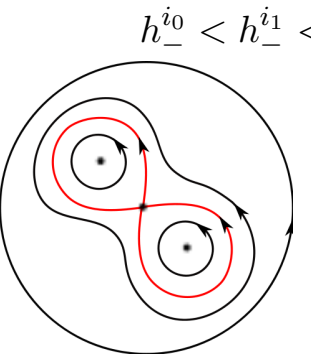

(c)

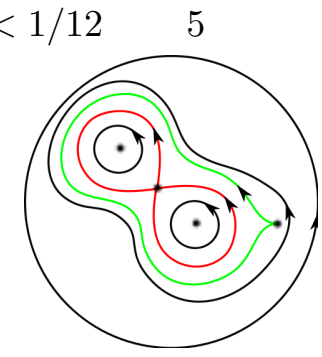

(d)

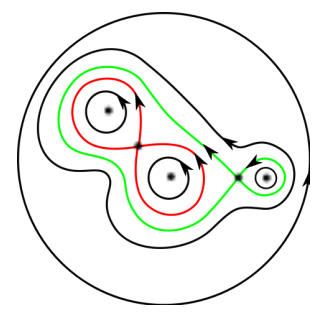

(e)

Fig. 19. Bifurcation of phase portraits of (2) in Case 4C (see Theorem 7(3)).

\begin{tabular}{clll} 
Figure & $\lambda$ & Hamiltonian & $\#_{s}$ \\
\hline (a) & $0 \leq \lambda<\lambda_{0}^{\mathcal{D}}$ & & 1 \\
(b) & $\lambda=\lambda_{0}^{\mathcal{D}}$ & $h^{i_{0}}=1 / 12$ & 2 \\
(c) & $\lambda_{0}^{\mathcal{D}}<\lambda<\lambda_{1}^{\mathcal{D}}$ & $h_{-}^{i_{0}}<1 / 12$ & 3 \\
(d) & $\lambda=\lambda_{1}^{\mathcal{D}}$ & $h_{-}^{i_{0}}<h^{i_{1}}=h^{i_{2}}=1 / 12$ & 5 \\
(e) & $\lambda<\lambda_{1}^{\mathcal{D}}$ & $h_{-}^{i_{0}}<h_{-}^{i_{1}}=h_{-}^{i_{2}}<1 / 12$ & 7
\end{tabular}

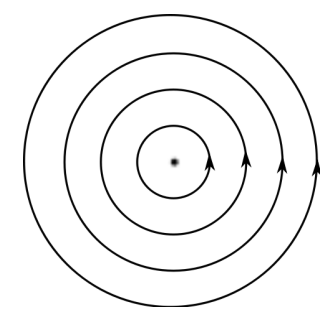

(a)

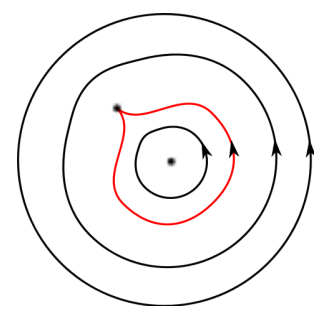

(b)

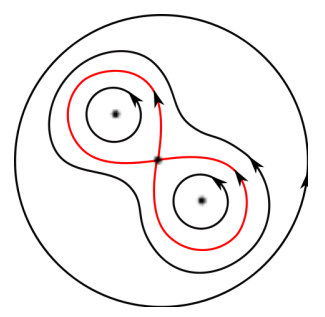

(c)

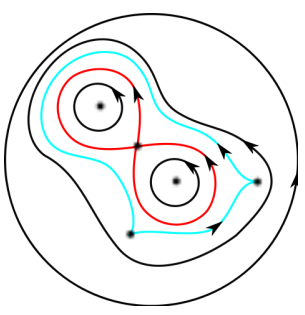

(d)

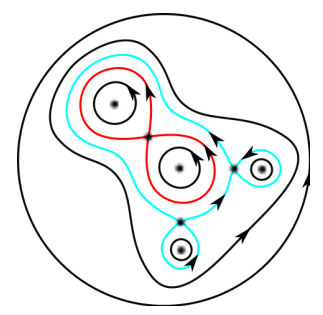

(e)

Fig. 20. Bifurcation of phase portraits of (2) in Case 4D (see Theorem 7(4)).

Proof. [Proof of Theorem 7 (4) and (5)] The bifurcation of the phase portraits with respect to increasing $\lambda$ in Cases $4 \mathrm{D}$ and $4 \mathrm{E}$ are analogous to the one in Case $4 \mathrm{~A}$, since the saddle points appearing on all the rays $\mathcal{R}_{i}$ correspond to the smallest ones $s_{-}^{i}, i=0,1,2$.

In Case 4D since $\left|\bar{B}_{i_{1}}\right|=\left|\bar{B}_{i_{2}}\right|$ we also have $\left|B_{i_{1}}(\lambda)\right|=\left|B_{i_{2}}(\lambda)\right|$ for all $\lambda$. Therefore in Case 4D first the singularities appear along $\mathcal{R}_{i_{0}}$, next the singularities appear simultaneously along $\mathcal{R}_{i_{1}}$ and $\mathcal{R}_{i_{2}}$, and the local phase portrait near $\mathcal{R}_{i_{2}}$ is an identic copy of the one near $\mathcal{R}_{i_{1}}$. The first three bifurcations are completely analogous to the ones in Case $4 \mathrm{~A}$, the last two bifurcations follow from an analysis based on the Hamiltonian.

In Case $4 \mathrm{E}$ since $\left|\bar{B}_{i_{0}}\right|=\left|\bar{B}_{i_{1}}\right|$ we also have $\left|B_{i_{0}}(\lambda)\right|=\left|B_{i_{1}}(\lambda)\right|$ for all $\lambda$. Therefore in Case $4 \mathrm{E}$ first the singularities appear along $\mathcal{R}_{i_{0}}$ and $\mathcal{R}_{1}$ simultaneously, where the local phase portraits near $\mathcal{R}_{0}$ and $\mathcal{R}_{1}$ are diffeomorphic copies; next the singularities appear along $\mathcal{R}_{i_{2}}$.

Proof. [Proof of Theorem 7(6) and 7(7)] The bifurcation of the phase portraits with respect to increasing $\lambda$ in Case $4 \mathrm{~F}$ is analogous to the one in Case $4 \mathrm{~B}$, since the saddle points appearing on the rays $\mathcal{R}_{i_{j}}, j=0,2$ correspond to the smallest ones $s_{-}^{i_{j}}, j=0,2$ and the saddle on $\mathcal{R}_{i_{1}}$ corresponds to the biggest one $s_{-}^{i_{1}}$. 


\begin{tabular}{clll} 
Figure & $\lambda$ & Hamiltonian & $\#_{s}$ \\
\hline (a) & $0 \leq \lambda<\lambda_{1}^{\mathcal{E}}$ & & 1 \\
(b) & $\lambda=\lambda_{1}^{\mathcal{E}}$ & $h^{i_{0}}=h^{i_{1}}=1 / 12$ & 3 \\
(c) & $\lambda_{1}^{\mathcal{E}}<\lambda<\lambda_{2}^{\mathcal{E}}$ & $h_{-}^{i_{0}}=h_{-}^{i_{1}}<1 / 12$ & 5 \\
(d) & $\lambda=\lambda_{2}^{\mathcal{E}}$ & $h_{\overline{-}}^{i_{0}}=h_{\overline{i_{1}}}^{i_{1}}<h^{i_{2}}=1 / 12$ & 6 \\
(e) & $\lambda>\lambda_{2}^{\mathcal{E}}$ & $h_{-}^{i_{0}}=h_{-}^{i_{1}}<h_{-}^{i_{2}}<1 / 12$ & 7
\end{tabular}

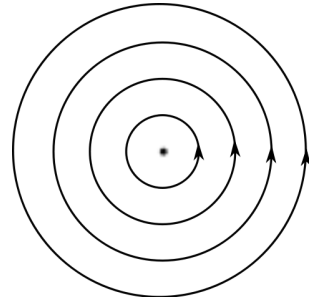

(a)

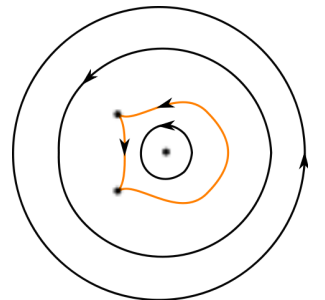

(b)

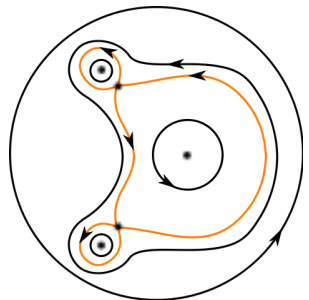

(c)

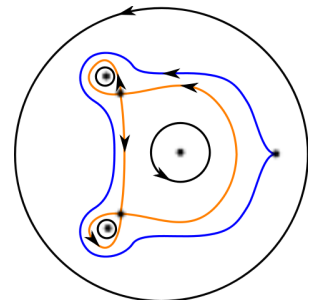

(d)

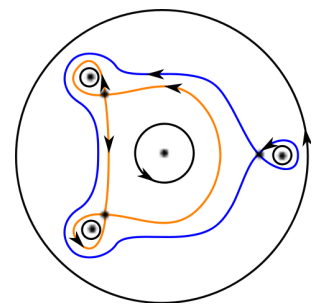

(e)

Fig. 21. Bifurcation of phase portraits of (2) in Case $4 \mathrm{E}$ (see Theorem 7(5)).

\begin{tabular}{llll} 
Figure & $\lambda$ & Hamiltonian & $\#$ \\
\hline (a) & $0 \leq \lambda<\lambda_{1}^{\mathcal{F}}$ & & 1 \\
(b) & $\lambda=\lambda_{1}^{\mathcal{F}}$ & $h^{i_{0}}=h^{i_{1}}=1 / 12$ & 3 \\
(c) & $\lambda_{1}^{\mathcal{F}}<\lambda<\lambda_{2}^{\mathcal{F}}$ & $h_{-}^{i_{0}}=h_{-}^{i_{1}}<1 / 12$ & 5 \\
(d) & $\lambda=\lambda_{2}^{\mathcal{F}}$ & $h_{-}^{i_{0}}=h_{-}^{i_{1}}<h^{i_{2}}=1 / 12$ & 6 \\
(e) & $\lambda_{2}^{\mathcal{F}}<\lambda<\lambda_{3}^{\mathcal{F}}$ & $h_{-}^{i_{0}}=h_{-}^{i_{1}}<h_{+}^{i_{2}}<h_{-}^{i_{2}}<1 / 12$ & 7 \\
(f) & $\lambda=\lambda_{3}^{\mathcal{F}}$ & $h_{-}^{i_{0}}=h_{-}^{i_{1}}=h_{+}^{i_{2}}<h_{-}^{i_{2}}<1 / 12$ & 7 \\
(g) & $\lambda>\lambda_{3}^{\mathcal{F}}$ & $h_{+}^{i_{2}}<h_{-}^{i_{0}}=h_{-}^{i_{1}}<h_{-}^{i_{2}}<1 / 12$ & 7
\end{tabular}

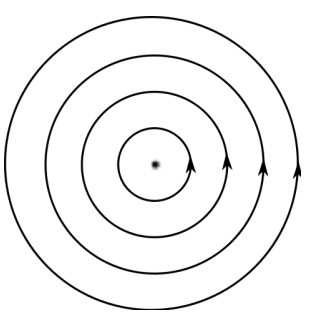

(a)

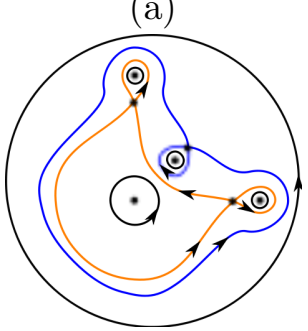

(e)

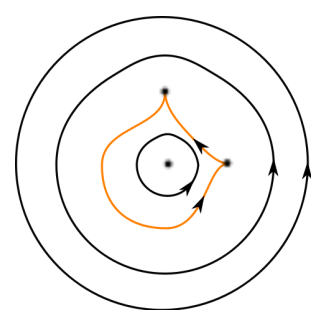

(b)

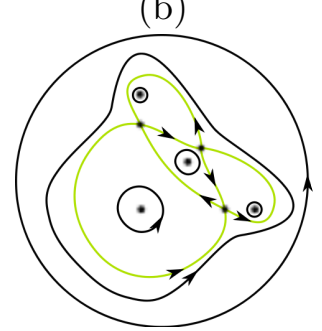

(f)

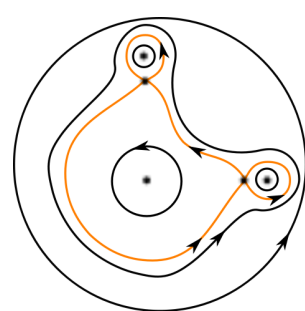

(c)

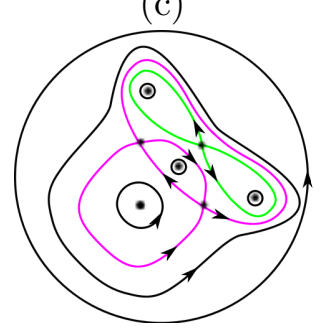

(g)

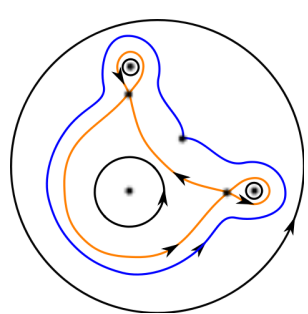

(d)

Fig. 22. Bifurcation of phase portraits of (2) in Case 4F (see Theorem 7(6)).

In Cases $4 \mathrm{~F}$ and $4 \mathrm{G}$ since $\left|\bar{B}_{i_{0}}\right|=\left|\bar{B}_{i_{1}}\right|$ we also have $\left|B_{i_{0}}(\lambda)\right|=\left|B_{i_{1}}(\lambda)\right|$, for all $\lambda$. Therefore in Case $4 \mathrm{~F}$ first the singularities appear along $\mathcal{R}_{i_{0}}$ and $\mathcal{R}_{i_{1}}$ simultaneously, where the local phase portraits near $\mathcal{R}_{i_{0}}$ and $\mathcal{R}_{i_{1}}$ are identic copies; next the singularities appear along $\mathcal{R}_{i_{2}}$.

In Case $4 \mathrm{G}\left(i_{0}, i_{1}, i_{2}\right)=(0,2,1)$ for $\left(\alpha_{1}, \beta_{1}\right)=(\sqrt{2} / 2,-\sqrt{2} / 4)$ and $\left(i_{0}, i_{1}, i_{2}\right)=(2,1,0)$ for $\left(\alpha_{2}, \beta_{2}\right)=$ 


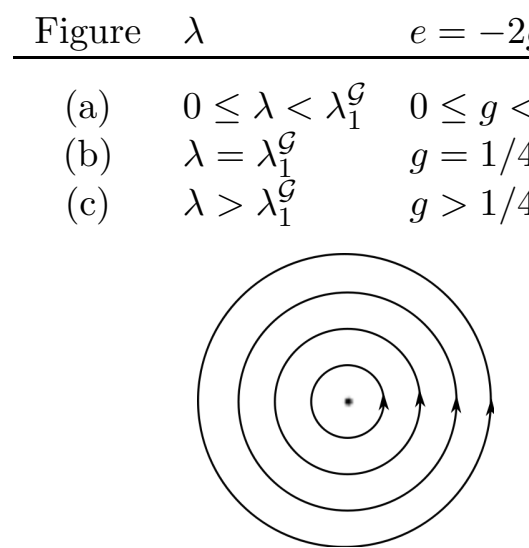

(a)

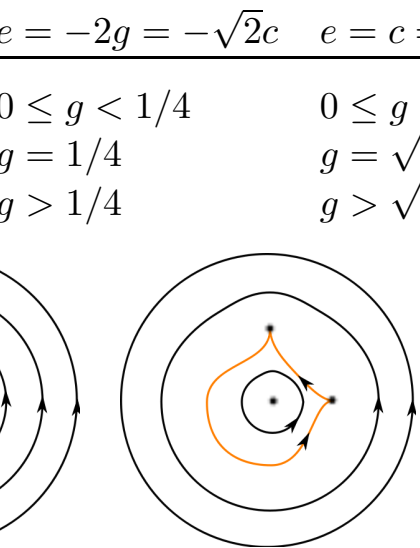

(b)

Fig. 23. Bifurcation of phase portraits of (2) in Case 4G (see Theorem $7(7)$ ).

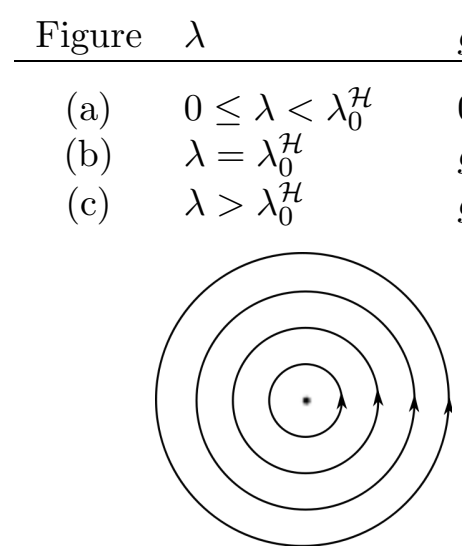

(a)

$g \quad$ Hamiltonian

$$
0 \leq g<2
$$$$
g=2
$$

$g>2$

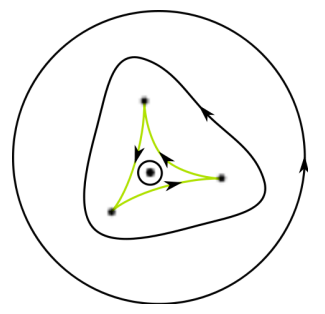

(b)

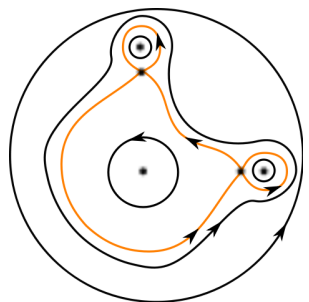

(c)

政

1

3

Fig. 24. Bifurcation of phase portraits of (2) in Case $4 \mathrm{H}$ (see Theorem $7(8)$ ).

$(\sqrt{2} / 2,-\sqrt{2} / 2)$. One can calculate that

$$
\bar{B}_{0}=\bar{B}_{2}=-\frac{1}{2} \text { for }(\alpha, \beta)=\left(\alpha_{1}, \beta_{1}\right) \text { and } \bar{B}_{2}=-\bar{B}_{1}=-\frac{\sqrt{3}}{3} \text { for }(\alpha, \beta)=\left(\alpha_{2}, \beta_{2}\right) .
$$

For both values of $(\alpha, \beta) \in \mathcal{G}$ one has that $\bar{B}_{i_{2}}=0$, and hence $B_{i_{2}}(\lambda)=0$ for all $\lambda$. Therefore no singularities appear on ray $\mathcal{R}_{i_{2}}$, for any value of the parameter $\lambda$. So the bifurcation of the phase portraits in Case $4 \mathrm{G}$ is analogous to the first three bifurcations in Case $4 \mathrm{~F}$, i.e., for $\lambda<\lambda_{2}^{\mathcal{F}}$.

Proof. [Proof of Theorem 7(8)] In Case $4 \mathrm{H}$ one has $\bar{B}_{0}=-\bar{B}_{1}=\bar{B}_{2}=-\frac{1}{3}>0$, and so

$$
B_{0}(\lambda)=-B_{1}(\lambda)=B_{2}(\lambda)=-\frac{\lambda}{3}, \text { for } \lambda \geq 0 .
$$

Furthermore all cusps appearing on $\mathcal{R}_{i}, i=0,1,2$ point outwards and the saddles appearing on $\mathcal{R}_{i}, i=0,1,2$ correspond to the smallest singularities $s_{-}^{i}, i=0,1,2$. As a consequence the singularities on $\mathcal{R}_{i}, i=0,1,2$ appear simultaneously and the local phase portraits near $\mathcal{R}_{i}, i=0,1,2$ are identic copies. Notice that in this case the original parameter is given by $(g, c, e)=(\lambda / 3,0,-\lambda / 3)$ and hence in Case $4 \mathrm{H}$ the systems are reversible. 


\section{Applications and Generalizations}

In this section we illustrate how the techniques developed for cubic irs-systems apply to polynomial differential systems of higher degree. Polynomial irs-systems of degree $n$ read in polar coordinates as

$$
\begin{aligned}
r^{\prime} & =\sum_{k=2}^{n-1} A_{k}(\theta) r^{k}, \\
\theta^{\prime} & =1+\sum_{k=1}^{n-2} B_{k}(\theta) r^{k}+r^{n-1},
\end{aligned}
$$

where $A_{k}(\theta)$ and $B_{k}(\theta)$ are homogeneous trigonometric polynomials of degree $k$. The singularities can be analyzed using Lemma 6.

When all but one $A_{k}$ and $B_{k}$ in (64) are identically zero, i.e., when $r^{\prime}=A_{i}(\theta) r^{i}, \theta^{\prime}=1+B_{j} r^{j}+r^{n-1}$, then the results for cubic irs-systems can directly be generalized. In section 6.1 we study the bifurcation diagram of such a 1-parameter subfamily of Hamiltonian quintic irs-systems.

In section 6.2 we study the bifurcation diagram of a more general 2-parameter subfamily of quintic irs-systems. In particular we explain how one can construct a polynomial that has a specified number of aligned singularities of a specified topological type and with the specified connections between them.

\subsection{The bifurcation diagram of a quintic system with a center at the origin and at the infinity}

In this section we consider Hamiltonian quintic systems that in polar coordinates write as (64) with $n=5$ and $A_{2} \equiv A_{4} \equiv 0$; then necessarily $B_{2} \equiv B_{4} \equiv 0$. As an example we take the 1-parameter subfamily depending on the parameter $a$

$$
X_{a}^{5} \leftrightarrow\left\{\begin{array}{l}
x^{\prime}=-y+a y^{3}-y\left(x^{2}+y^{2}\right)^{2} \\
y^{\prime}=x+a x^{3}+x\left(x^{2}+y^{2}\right)^{2}
\end{array}\right.
$$

which in polar coordinates writes as

$$
\begin{aligned}
& r^{\prime}=a \sin \theta \cos \theta r^{3}, \\
& \theta^{\prime}=1+a\left(-1+2 \cos ^{2} \theta\right) r^{2}+r^{4} .
\end{aligned}
$$

The Hamiltonian is given by $H(x, y)=\frac{1}{2}\left(x^{2}+y^{2}\right)+\frac{1}{4} a\left(x^{4}-y^{4}\right)+\frac{1}{6}\left(x^{2}+y^{2}\right)^{3}$. Since $X_{a}^{5}$ is invariant under $(t, x, y) \mapsto(-t,-x, y)$ (resp. $(t, x, y) \mapsto(-t,-x, y))$, its phase portrait is symmetric with respect to the horizontal (resp. vertical) axis reversing time. For $|a|<2$ the vector field $X_{a}^{5}$ has a global center at the origin. For $|a|=2$ the vector field has two singularities with $r=1$ on the horizontal (resp. vertical) axis symmetric for $a<0$ (resp. $a>0$ ) with Hamiltonian value $h=1 / 6$. For $|a|>2$ the vector field has four singularities with $r=r_{ \pm}$on the horizontal (resp. vertical) axis symmetric for $a<0$ (resp. $a>0$ ) where $r_{ \pm}^{2}=\left(|a| \pm \sqrt{a^{2}-4}\right) / 2$. Using analogous techniques as in section 5 we obtain the bifurcation diagram and the phase portrait of system (65) in terms of the parameter $a$, see Figure 25.

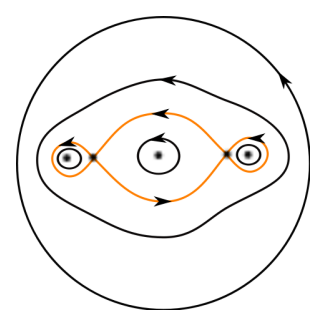

$a<-2$

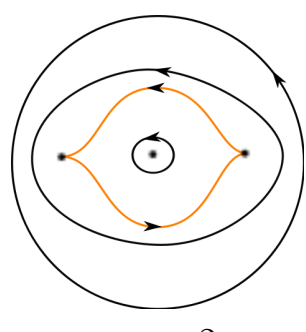

$a=-2$

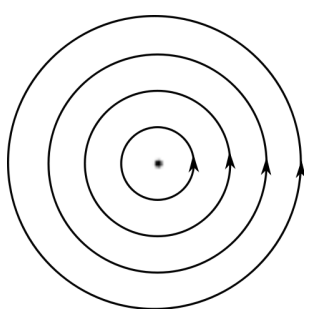

$-2<a<2$

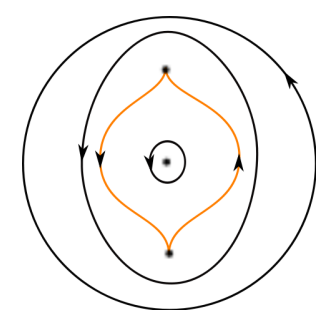

$a=2$

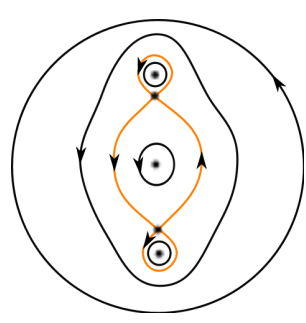

$2<a$

Fig. 25. Bifurcation diagram and phase portraits of (65). 


\subsection{How to construct a system with a given phase portrait}

In this section we derive some sufficient conditions for polynomial irs-systems whose phase portrait looks like the ones of Figure 27. Observing that all these phase portraits have a center at the origin and at infinity, both with the same orientation, all these phase portraits are reversible having five aligned singularities, we look for a quintic planar system satisfying these conditions

$$
\begin{aligned}
& \dot{x}=-y+a_{11} x y+a_{21} x^{2} y+a_{03} y^{3}+a_{31} x^{3} y+a_{13} x y^{3}-y\left(x^{2}+y^{2}\right)^{2}, \\
& \dot{y}=x+b_{20} x^{2}+b_{02} y^{2}+b_{30} x^{3}+b_{12} x y^{2}+b_{40} x^{4}+b_{22} x^{2} y^{2}+b_{04} y^{4}+x\left(x^{2}+y^{2}\right)^{2} .
\end{aligned}
$$

As for cubic irs-systems, the phase portraits in Figure 27 can be generated by perturbing the corresponding degenerate cases drawn in Figure 26 in the appropriate way. In order that system (66) has two double singularities on the positive horizontal axis, e.g., at $\mathbf{s}_{1} \equiv(1 / 2,0)$ and $\mathbf{s}_{2} \equiv(2,0)$, it is necessary that

$$
b_{02}=b_{12}=b_{22}=b_{04}=0, \quad b_{20}=b_{40}=-5, \quad \text { and } \quad b_{30}=\frac{33}{4} .
$$

Then by Lemma 6 with $k=2$ and $l=1$ it is sufficient to pick some parameter values $\left(a_{11}, a_{21}, a_{31}\right)$ such that the sign of $\gamma \equiv \gamma\left(\mathbf{s}_{i}\right), i=1,2$ leads to the appropriate orientation of the cusps $\mathbf{s}_{i}, i=1,2$ as in Figure 26 , where

$$
\begin{aligned}
& \gamma\left(\mathbf{s}_{1}\right)=\frac{1}{36}\left(-17+8 a_{11}+4 a_{21}+2 a_{31}\right), \text { and } \\
& \gamma\left(\mathbf{s}_{2}\right)=\frac{4}{9}\left(-17+2 a_{11}+4 a_{21}+8 a_{31}\right) .
\end{aligned}
$$

From these expressions it follows that the orientation of the cusps can independently be changed as in Figure 26 when we restrict our study to

$$
a_{21}=a_{11}=0, \quad a_{31}=a \in \mathbb{R} \backslash\{17 / 8,17 / 2\} .
$$

In order that system (66) with (67) and (68) has no singularities outside the horizontal axis, it is necessary that $a_{03}=a_{13}=0$ and the following 1-parameter family of quintic irs-systems is induced

$$
X_{a}^{R, 5} \leftrightarrow\left\{\begin{array}{l}
x^{\prime}=-y+a x^{3} y-y\left(x^{2}+y^{2}\right)^{2}, \\
y^{\prime}=x-5 x^{2}+\frac{33}{4} x^{3}-5 x^{4}+x\left(x^{2}+y^{2}\right)^{2} .
\end{array}\right.
$$

An analogous reasoning as in section 5 shows that the bifurcation diagram of the global phase portraits of $X_{a}^{R, 5}$ in terms of $a$ is given by Figure 26. Notice that we here have not included the phase portraits corresponding to the bifurcation values $a=17 / 8$ and $a=17 / 2$. These phase portraits can be obtained by a continuity argument. However the goal of this section is to find explicit irs-systems having phase portraits as in Figure 27.

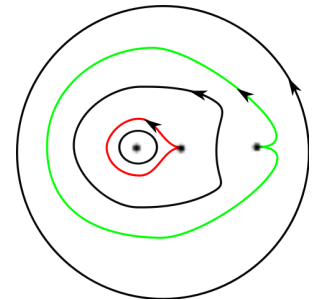

(a) $a<17 / 8$

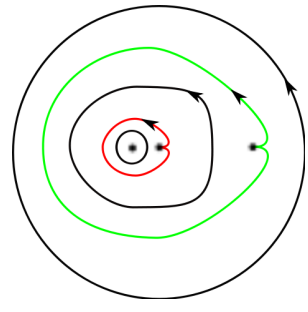

(b) $17 / 8<a<17 / 2$

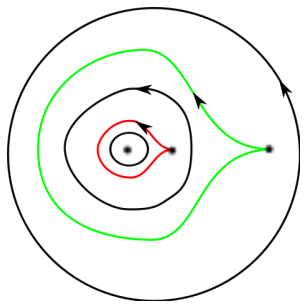

(c) $a>17 / 2$

Fig. 26. Phase portraits of Eq. (69).

Analogously one constructs a 2-parameter family $X_{(a, \varepsilon)}^{R, 5}$ of quintic irs-systems having four singularities on the positive horizontal axis, say at $(2,0),(2+\varepsilon, 0),(1 / 2,0)$ and $(1 /(2+\varepsilon), 0)$ with

$$
X_{(a, \varepsilon)}^{R, 5} \leftrightarrow\left\{\begin{array}{l}
x^{\prime}=-y+a x^{3} y-y\left(x^{2}+y^{2}\right)^{2} \\
y^{\prime}=x+b(\varepsilon) x^{2}+c(\varepsilon) x^{3}+b(\varepsilon) x^{4}+x\left(x^{2}+y^{2}\right)^{2}
\end{array}\right.
$$


where

$$
b(\varepsilon)=-\frac{(4+\varepsilon)(5+2 \varepsilon)}{2(2+\varepsilon)} \quad \text { and } \quad c(\varepsilon)=\frac{33+24 \varepsilon+5 \varepsilon^{2}}{2(2+\varepsilon)} \text { for } \varepsilon>0 .
$$

Clearly $X_{(a, 0)}^{R, 5} \equiv X_{a}^{R, 5}$. Again by Lemma 6 and since there are no other singularities present outside the horizontal axis, it follows that for each $a$ there exists $\varepsilon_{0}(a)>0$ such that for all $0<\varepsilon<\varepsilon_{0}(a)$ the phase portrait of $X_{(a, \varepsilon)}^{R, 5}$ coincides globally with the corresponding one in Figure 27. In particular $\varepsilon_{0}(a)$ is an algebraic function that is implicitly defined. For example $\varepsilon_{0}(1)=+\infty, \varepsilon_{0}(5)$ and $\varepsilon_{0}(30)$ are the positive zeros of $\varepsilon^{4}+3 \varepsilon^{3}-6 \varepsilon^{2}-28 \varepsilon-23$ and $\varepsilon^{4}+8 \varepsilon^{3}+24 \varepsilon^{2}+2 \varepsilon-43$ respectively. Approximately these values are $\varepsilon_{0}(5) \approx 2.991961290$ and $\varepsilon_{0}(30) \approx 1.096040970$.

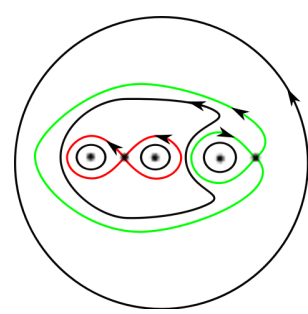

(a) $a<17 / 8$

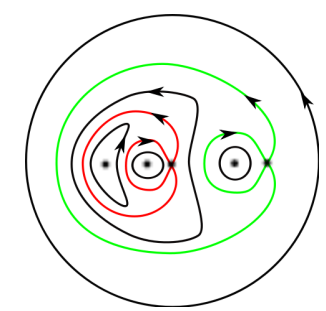

(b) $17 / 8<a<17 / 2$

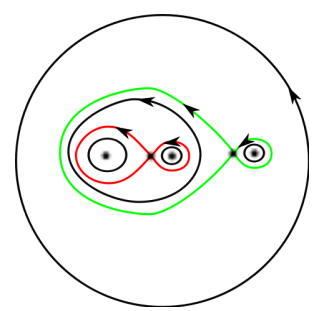

(c) $a>17 / 2$

Fig. 27. Phase portraits of Eq. (70) for $\varepsilon$ small enough.

From this study we can pick an infinite number of values $(a, \epsilon)$ to give an explicit irs-system having the phase portrait described in Figure 27(a),(b) respectively (c), for instance the following irs-system with $a=1, a=5$ respectively $a=30$ :

$$
\begin{aligned}
& x^{\prime}=-y+a x^{3} y-y\left(x^{2}+y^{2}\right)^{2}, \\
& y^{\prime}=x-\frac{35}{6} x^{2}+\frac{31}{3} x^{3}-\frac{35}{6} x^{4}+x\left(x^{2}+y^{2}\right)^{2} .
\end{aligned}
$$

\section{Acknowledgments}

The authors are partially supported by the MICINN/FEDER grant with reference number MTM200803437 and by the AGAUR grant with reference number 2009SGR 410. Furthermore the first author is partially supported by the grant Juan de la Cierva with reference number JCI-2007-49-764.

\section{References}

Bautin, N. N. [1954] "On the number of limit cycles which appear with the variation of coefficients from an equilibrium position of focus or center type," American Math. Soc. Translation 1954, 19.

Blows, T. R. \& Rousseau, C. [1993] "Bifurcation at infinity in polynomial vector fields," J. Differential Equations 104, 215-242.

Buzzi, C. A., Llibre, J. \& Medrado, J. C. R. [2009] "Phase portraits of reversible linear differential systems with cubic homogeneous polynomial nonlinearities having a non-degenerate center at the origin," Qual. Theory Dyn. Syst. 7, 369-403.

Caubergh, M. \& Dumortier, F. [2004] "Hopf-Takens bifurcations and centres," J. Differential Equations 202, $1-31$.

Caubergh, M. \& Dumortier, F. [2008] "Hilbert's 16th problem for classical Liénard equations of even degree," J. Differential Equations 244, 1359-1394.

Dulac, H. [1908] "Détermination et integration d'une certaine classe d'équations différentielle ayant par point singulier un centre," Bull. Sci. Math. Sér. (2) 32, 230-252. 
Dumortier, F., Llibre, J. \& Artés, J. C. [2006] Qualitative theory of planar differential systems, Universitext (Springer-Verlag, Berlin), ISBN 3-540-32893-9.

Gasull, A., Guillamon, A. \& Mañosa, V. [2000] "Phase portrait of Hamiltonian systems with homogeneous nonlinearities," Nonlinear Anal. 42, 679-707.

Gasull, A. \& Torregrosa, J. [2001] "A new approach to the computation of the Lyapunov constants," Comput. Appl. Math. 20, 149-177, the geometry of differential equations and dynamical systems.

Gonsales, È. [1984] "Generic properties of Lyapunov exponents of equations of arbitrary order," Mat. Zametki 36, 201-211.

Guillamon, A. \& Pantazi, C. [2008] "Phase portraits of separable hamiltonian systems," Preprint.

Huang, W. \& Liu, Y. [2004a] "Bifurcations of limit cycles from infinity for a class of quintic polynomial system," Bull. Sci. Math. 128, 291-302.

Huang, W. \& Liu, Y. [2004b] "A polynomial differential system with nine limit cycles at infinity," Comput. Math. Appl. 48, 577-588.

Kapteyn, W. [1911] "On the midpoints of integral curves of differential equations of the first degree," Nederl. Akad. Wetensch. Verslag. Afd. Natuurk. Konikl. Nederland, 1446-1457.

Kapteyn, W. [1912] "New investigations on the midpoints of integrals of differential equations of the first degree," Nederl. Akad. Wetensch. Verslag Afd. Natuurk. 20, 1354-1365.

Liu, Y. \& Chen, H. [2002] "Stability and bifurcations of limit cycles of the equator in a class of cubic polynomial systems," Comput. Math. Appl. 44, 997-1005.

Liu, Y. \& Huang, W. [2006] "Seven large-amplitude limit cycles in a cubic polynomial system," Internat. J. Bifur. Chaos Appl. Sci. Engrg. 16, 473-485.

Lloyd, N. G. \& Pearson, J. M. [1999] "Bifurcation of limit cycles and integrability of planar dynamical systems in complex form," J. Phys. A 32, 1973-1984.

Luca, S., Dumortier, F., Caubergh, M. \& Roussarie, R. [2009] "Detecting alien limit cycles near a Hamiltonian 2-saddle cycle," Discrete Contin. Dyn. Syst. 25, 1081-1108.

Markus, L. [1960] "Quadratic differential equations and non-associative algebras," Contributions to the theory of nonlinear oscillations, Vol. V (Princeton Univ. Press, Princeton, N.J.), pp. 185-213.

Neumann, D. A. [1975] "Classification of continuous flows on 2-manifolds," Proc. Amer. Math. Soc. 48, 73-81.

Peixoto, M. [1971] "Sur la classification des équations différentielles," C. R. Acad. Sci. Paris Sér. A-B 272, A262-A265.

Schlomiuk, D. [1993a] "Algebraic and geometric aspects of the theory of polynomial vector fields," Bifurcations and periodic orbits of vector fields (Montreal, PQ, 1992) (Kluwer Acad. Publ., Dordrecht), pp. $429-467$.

Schlomiuk, D. [1993b] "Algebraic particular integrals, integrability and the problem of the center," Trans. Amer. Math. Soc. 338, 799-841.

Vulpe, N. I. \& Sibirskiı̌, K. S. [1988] "Centro-affine invariant conditions for the existence of a center of a differential system with cubic nonlinearities," Dokl. Akad. Nauk SSSR 301, 1297-1301.

Zhang, Q. \& Liu, Y. [2006] "A cubic polynomial system with seven limit cycles at infinity," Appl. Math. Comput. 177, 319-329.

Zhang, Q. \& Liu, Y. [2007] "A quintic polynomial differential system with eleven limit cycles at the infinity," Comput. Math. Appl. 53, 1518-1526.

Zhang, Q., Liu, Y. \& Chen, H. [2006] "Bifurcation at the equator for a class of quintic polynomial differential system," Appl. Math. Comput. 181, 747-755.

Żołądek, H. [1994a] "On a certain generalization of Bautin's theorem," Nonlinearity 7, 273-279.

Żołądek, H. [1994b] "Quadratic systems with center and their perturbations," J. Differential Equations 109, 223-273. 